\title{
Superconformal index, BPS monodromy and chiral algebras
}

\author{
Sergio Cecotti, ${ }^{a}$ Jaewon Song, ${ }^{b}$ Cumrun $\operatorname{Vafa}^{c}$ and Wenbin $\operatorname{Yan}^{c, d, e}$ \\ ${ }^{a}$ Scuola Internazionale Superiore di Studi Avanzati, \\ via Bonomea 265, 34100 Trieste, Italy \\ ${ }^{b}$ Department of Physics, University of California, \\ 9500 Gilman Dr, La Jolla, CA 92093, U.S.A. \\ c Jefferson Physical Laboratory, Harvard University, \\ 17 Oxford Street, Cambridge, MA 02138, U.S.A. \\ ${ }^{d}$ Center for Mathematical Sciences and Applications, Harvard University, \\ 20 Garden Street, Cambridge, MA 02138, U.S.A. \\ e Walter Burke Institute for Theoretical Physics, California Institute of Technology, \\ 1200 E. California Blvd, Pasadena, CA 91125, U.S.A. \\ E-mail: cecotti@sissa.it, jsong@physics.ucsd.edu, \\ vafa@physics.harvard.edu, wbyan@cmsa.fas.harvard.edu
}

ABSTRACT: We show that specializations of the $4 \mathrm{~d} \mathcal{N}=2$ superconformal index labeled by an integer $N$ is given by $\operatorname{Tr} \mathcal{M}^{N}$ where $\mathcal{M}$ is the Kontsevich-Soibelman monodromy operator for BPS states on the Coulomb branch. We provide evidence that the states enumerated by these limits of the index lead to a family of $2 \mathrm{~d}$ chiral algebras $\mathcal{A}_{N}$. This generalizes the recent results for the $N=-1$ case which corresponds to the Schur limit of the superconformal index. We show that this specialization of the index leads to the same integrand as that of the elliptic genus of compactification of the superconformal theory on $S^{2} \times T^{2}$ where we turn on $\frac{1}{2} N$ units of $\mathrm{U}(1)_{r}$ flux on $S^{2}$.

KeYwords: Conformal Field Theory, Supersymmetric Gauge Theory

ArXiv EPRINT: 1511.01516 


\section{Contents}

1 Introduction 1

2 Review 2d case $\quad 3$

3 BPS monodromy and 4d index $\quad 4$

3.1 The Hopf surface 5

3.2 A specialization of the 4d index 6

$\begin{array}{lll}3.3 & \text { BPS mondromy and the specialized index } & 7\end{array}$

4 Lagrangian theories $\quad 8$

4.1 Specialized index for the Lagrangian theory 9

$\begin{array}{lll}\text { 4.1.1 Insertion of Wilson loop line operators } & 10\end{array}$

$\begin{array}{lll}4.1 .2 & \text { Free hypermultiplets } & 10\end{array}$

$\begin{array}{lll}4.1 .3 & \text { Free vector multiplets } & 12\end{array}$

$\begin{array}{lll}4.2 & \text { Chiral algebras } & 14\end{array}$

$\begin{array}{lll}4.2 .1 & \text { Free theories } & 15\end{array}$

$\begin{array}{lll}4.2 .2 & \text { Interacting theory } & 17\end{array}$

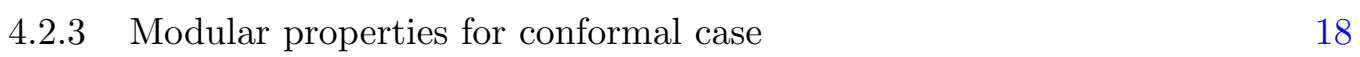

$\begin{array}{lll}4.3 & \text { Lagrangian examples } & 19\end{array}$

$\begin{array}{ll}\text { 4.3.1 Abelian theories with matter } & 19\end{array}$

$\begin{array}{lll}\text { 4.3.2 } & \mathrm{U}(1) \text { theory with } N_{f} \text { hypermultiplets } & 21\end{array}$

$\begin{array}{ll}\text { 4.3.3 Pure } \mathrm{SU}(2) & 23\end{array}$

$\begin{array}{lll}\text { 4.3.4 } \mathrm{SU}(2) \text { with } N_{f}=4 & 24\end{array}$

$5 T^{\mathbf{2}} \times S^{\mathbf{2}}$ compactifications $\quad \mathbf{2 5}$

$5.12 \mathrm{~d} \mathcal{N}=(0,2)$ theory from twisted compactification on $S^{2} \quad 26$

5.2 Central charge 28

$5.3 T^{2} \times S^{2}$ vs. $S^{1} \times S^{3}$ partition functions $\quad 29$

$6 \quad$ BPS monodromy and the specialized index $\quad 32$

6.1 Free hypers and hypers coupled to abelian gauge groups 33

6.2 Physical arguments for models having Lagrangian formulation 34

6.2.1 The physical picture at extreme weak coupling 36

$\begin{array}{ll}\text { 6.2.2 Decoupling of infinitely massive dyons and S-duality } & 39\end{array}$

6.2.3 Product identities for dyonic towers 40

6.3 $\mathrm{SU}(2) \mathrm{SQCD}$ with $N_{f}=4$ and $S$-duality $\quad 42$

6.4 A non-Lagrangian example 45

$\begin{array}{ll}6.5 & \text { Generalities on monodromy traces }\end{array}$ 
7 Monodromies and $2 d$ chiral algebras of Argyres-Douglas theories

7.1 Insertion of line operators and characters of chiral algebras $\quad 52$

$\begin{array}{lll}7.2 & \left(A_{1}, A_{2}\right) \text { AD theory } & 52\end{array}$

$7.3\left(A_{1}, A_{2 n}\right)$ AD theories $\quad 56$

$7.4\left(A_{1}, A_{2 n+1}\right)$ AD theories $\quad 57$

$\begin{array}{lll}\text { 7.4.1 The special }\left(A_{1}, A_{3}\right) \text { model } & 58\end{array}$

$7.5\left(A_{1}, D_{2 n+1}\right)$ AD theories $\quad 59$

$7.6\left(A_{1}, D_{2 n}\right)$ AD theories 61

8 Concluding remarks $\quad 62$

A Partial topological twisting and effective 2d central charges $\quad 63$

$\begin{array}{lll}\text { A.1 Twisting of the free theory } & 64\end{array}$

A.2 Central charge of the 2d effective theory 66

$\begin{array}{ll}\text { B Superconformal index for Lagrangian theories } & 67\end{array}$

C The tool box for monodromy traces $\quad 70$

$\begin{array}{lll}\text { C.1 } & q \text {-hypergeometric series } & 70\end{array}$

$\begin{array}{lll}\text { C.2 The Moyal approach and Hardy norms } & 72\end{array}$

$\begin{array}{lll}\text { C.3 The Moyal function of the operator } \mathcal{M}(q)^{N} & 74\end{array}$

C.4 Asymptotic analysis of $\operatorname{Tr} \mathcal{M}(q)^{N}$ : the effective central charge $c_{\text {eff }} \quad 75$

$\begin{array}{ll}\text { D Some } q \text {-hypergeometric identities } & \mathbf{7 9}\end{array}$

$\begin{array}{lll}\text { D.1 Expansion of } 1 / \Theta \text { in Ramanujan's partial thetas } & 79\end{array}$

D.2 $\operatorname{Tr}\left[\mathcal{M}(q)^{-1} X_{\gamma}\right]$ for the $\left(A_{1}, A_{2}\right)$ model $\quad 80$

D.3 $\operatorname{Tr} \mathcal{M}(q)^{-1}$ for $\left(A_{1}, A_{2 n}\right)$ is the character of the $(2,2 n+3)$ minimal model 82

D.4 $\operatorname{Tr} \mathcal{M}(q)^{-1}$ for the $\left(A_{1}, A_{2 n+1}\right)$ AD model 83

\section{Introduction}

Four dimensional superconformal field theories (SCFT's) with $\mathcal{N}=2$ supersymmetry have common features with the simpler 2d SCFT's with $\mathcal{N}=(2,2)$ supersymmetry: upon deformations away from the conformal point, both typically lead to Bogomol'nyi-PrasadSommerfield (BPS) states whose mass is given by absolute value of a central charge in the SUSY algebra which is a complex number. Both cases undergo wall-crossing where the number of BPS states change [1-3]. Despite the wall-crossing, one can form an invariant from monodromy operators, ordered by the phases of the central charge, whose invariance under wall crossing characterizes the nature of jumps in the spectrum of BPS states $[1,4,5]$. The monodromy operator is invariant up to conjugation and so its eigenvalues, captured by the trace of its integer powers, lead to invariants of the SCFT. It was shown in $[1,4]$ that the eigenvalues of the monodromy matrix capture $\exp (2 \pi i Q)$ where $Q$ is the $\mathrm{U}(1)_{R}$ charge 
of the ground states of the theory. This result motivated the parallel question in the $4 \mathrm{~d}$ case [6] where the trace of the powers of the monodromy operator $\mathcal{M}(q)$ were computed. It was found that if the $\mathrm{U}(1)_{r}$ charges of the conformal theory are integer multiples of $1 / k$, then the monodromy operator to the power $k$ acts as the identity, ${ }^{1}$ very much as in the $2 \mathrm{~d}$ case, and an explanation for this phenomenon was provided. More surprising was that the trace of powers of the monodromy operator $\mathcal{M}(q)$ (including fractional powers of $\mathcal{M}(q)$ when the theory had additional discrete R-symmetries) in many cases were related to characters of rational $2 \mathrm{~d}$ conformal theories including minimal models and coset models. Furthermore it was found that insertion of line operators in the monodromy trace acts by changing the characters of the $2 \mathrm{~d}$ conformal theory. In addition, it was suggested in [7] that an integer sequence of specializations of the superconformal index should lead to the trace of various powers of the monodromy. In later work [8] it was found that, by considering operators contributing to the Schur limit of the superconformal index, one obtains chiral algebras in $2 \mathrm{~d}$, with a very specific central charge: $c^{2 d}=-12 c^{4 d}$, where $c^{2 d}$ is the $2 \mathrm{~d}$ central charge of the Virasoro algebra and $c^{4 d}$ is the $c$-function of the $4 \mathrm{~d}$ SCFT. Motivated by this, in a recent work [9] it was shown that the Schur index specialization is equal to the trace of the inverse monodromy operator $\operatorname{Tr} \mathcal{M}(q)^{-1}$.

One main motivation for this paper is to generalize this for arbitrary powers of $N$ by revisiting the specialization of the superconformal index suggested in [7]. We argue that a modification of that proposal identifies the trace of the $N$-th power of the monodromy operator $\operatorname{Tr} \mathcal{M}(q)^{N}$ as the superconformal index $\mathcal{I}(q, p, t)$ with $t=q p^{N+1}$ and $p \rightarrow \exp (2 \pi i)$ extending the $N=-1$ case. The $N=-1$ is the special case where the index is automatically $p$-independent. For other values of $N$ one needs to insert some operators for the superconformal index to lead to a non-vanishing finite answer. The reason for this is that typically there are some elements of chiral algebra which correspond to either non-compact bosons, or fermionic zero modes which, if not absorbed, would make the index diverge or vanish. We provide evidence for the identification of this limit of the superconformal index with $\operatorname{Tr} \mathcal{M}(q)^{N}$ by providing general arguments and also by showing explicitly that it works for Lagrangian theories. Moreover we present evidence for the existence of a $2 \mathrm{~d}$ chiral algebra $\mathcal{A}_{N}$ and construct this chiral algebra in the limit of the extreme weak coupling. For non-Lagrangian theories we broaden the scope of examples studied in [6] and identify the corresponding chiral algebras for a number of Argyres-Douglas type theories. There are two notions of central charge one can associate to these chiral algebras: one is the Casimir of the vacuum character, which leads to

$$
c^{2 d}=12 N c^{4 d},
$$

generalizing the Schur index case with $N=-1$. The other one is the growth in the number of chiral algebra elements of given level which leads (when $c^{4 d}-a^{4 d}>0$ which is the more typical case)

$$
c_{\text {eff }}^{2 d}= \begin{cases}-48 N\left(c^{4 d}-a^{4 d}\right) & \text { for } N<0 \\ 12 N c^{4 d} & \text { for } N>0 .\end{cases}
$$

\footnotetext{
${ }^{1}$ More precisely it commutes with the line operators.
} 
The equality $c^{2 d} \equiv c_{\text {eff }}^{2 d}$ for $N>0$ is consistent with $\operatorname{Tr} \mathcal{M}(q)^{N}$ being a character of a unitary 2d CFT in this case, as found in various examples [6].

We also consider partial topological twisting of $4 \mathrm{~d} \mathcal{N}=2$ superconformal theory on $S^{2}$ with $\frac{1}{2} N$ units of $\mathrm{U}(1)_{r}$ flux. This leads to $(0,2)$ supersymmetric theories in 2 d. Studying the $(0,2)$ elliptic genus of these theories leads essentially to the same expression for the integrand as in the above specialization of the index. However, while one uses the JeffreyKirwan residue prescription to compute the elliptic genus, in the index case the integral is over the unit circle. In addition one finds that (for $N>0$ ) the central charge of the $2 \mathrm{~d}$ theory is $12 N c^{4 d}$. For $N<0$ one obtains instead the $c_{\text {eff }}^{2 d}$ as the central charge of the resulting $2 \mathrm{~d}$ theory.

It turns out that there are two competing versions of the Kontsevich-Soibelman operator: one involves the compact version of the quantum dilogarithm, which is the main focus of the present paper, and the other one uses the non-compact version of it. The trace of the $N$-th powers of the non-compact version of the monodromy is related to compactifying the 4d theory on $S^{1} \times S^{3}$ where as we go around the circle $S^{1}$ we twist by $\exp (2 \pi i N(r-R))$, and computes its partition function on the squashed $S^{3}$. This is the connection proposed in [6] for the non-compact version.

The organization of this paper is as follows: in section 2 we review the relation between the elliptic genus of $(2,2)$ theories and the BPS monodromy. In section 3 we discuss the $4 \mathrm{~d}$ case and outline the argument for the connection between the BPS monodromy and specializations of the superconformal index as well as its compactification on $S^{1}$ twisted by $\mathrm{U}(1)_{r}$ charge. In section 4 we discuss the $4 \mathrm{~d} \mathcal{N}=2$ models with a Lagrangian description. In section 5 we study the compactification of the theory on $S^{2} \times T^{2}$ with $\mathrm{U}(1)_{r}$ flux through $S^{2}$ for the Lagrangian models. In section 6 we discuss how the traces of the monodromy operators are formulated and computed. In section 7 we give a number of examples for Argyres-Douglas theories. In section 8 we present some concluding thoughts. Some technical discussions are postponed to the appendices.

\section{Review 2d case}

Consider a $(2,2)$ superconformal theory in 2 dimensions. Let $J_{0}, L_{0}$ denote the left-moving $\mathrm{U}(1)_{L}$ R-charge and left-moving Hamiltonian $L_{0}=H-P$ in the NS sector. Let $H_{L}=$ $L_{0}-\frac{1}{2} J_{0}$ be the Hamiltonian in the R-sector, and similar quantities for the right-movers. The superconformal index, which can be viewed as the Witten index, in this case is known as the elliptic genus and is given by

$$
Z_{\text {ell }}(z, q)=\operatorname{Tr}(-1)^{F} z^{J_{0}} q^{H_{L}} \bar{q}^{H_{R}} .
$$

The elliptic genus is independent of $\bar{q}$ since $H_{R}=\left\{Q_{R}, Q_{R}^{\dagger}\right\}$ and $Q_{R}$ commutes with $J_{0}, H_{L}$. Let us consider the specialization of this elliptic genus to

$$
z=\exp (2 \pi i N)
$$

where $N$ is an integer. Then $Z_{\text {ell }}$ will become independent of $q$ because in this limit also $Q_{L}$ commutes with $z^{J_{0}}=\exp \left(2 \pi i N J_{0}\right)$ (as it carries $J_{0}$ charge 1 ). So in this limit the 
partition function becomes just a number. Moreover since it is independent of $q$ and $\bar{q}$ we can compute it in the limit $q, \bar{q} \rightarrow 0$ which implies that it is the index restricted to Ramond ground states:

$$
I_{N}=Z_{\text {ell }}(\exp (2 \pi i N), q)=\underset{\text { states }}{\operatorname{ground}}(-1)^{F} \exp \left(2 \pi i N J_{0}\right)
$$

From this definition it is not a priori clear why $I_{N}$ has to be an integer, but it is. To see this [10] note that, by a modular transformation of the torus, this computation is the same as counting the Ramond ground states where the space is twisted by $\exp \left(2 \pi i N J_{0}\right)$. Note that if the spectrum of $J_{0}$ is rational of the form $r / k$ then

$$
I_{N}=I_{N+k}
$$

so the specialized indices $I_{N}$ compute only a finite number of independent invariants.

So far we have been studying the conformal point. Now suppose that the SCFT admits a deformation with $m$ supersymmetric vacua having a mass-gap. This massive deformation of $(2,2)$ theory will have some BPS solitons $\alpha_{i j}$ with a complex valued central charge $Z_{\alpha}$ connecting the $i$-th vacuum to the $j$-th one. Associate an $m \times m$ upper triangular matrix $M_{\alpha_{i j}}$ with 1 along the diagonal and $i j$ entry $n_{i j}$, which is a signed version of the number of solitons from $i$-th vacuum to the $j$-th (see [1] for details). Order the BPS solitons according to the phase $\arg Z_{\alpha}$ and consider the ordered product

$$
M=\prod_{i j} T\left(M_{\alpha_{i j}}\right)
$$

where $T$ denotes the phase ordering. When one changes the parameters of the massive theory the number of BPS states change in such a way that $M$ simply gets conjugated. In particular the traces of all its powers are invariant. Moreover, as shown in [1]

$$
\operatorname{Tr} M^{N}=I_{N}=\operatorname{Tr}_{\text {states }}^{\text {ground }}(-1)^{F} \exp \left(2 \pi i N J_{0}\right) .
$$

In other words, the specialization of the superconformal index (2.3) has an extension away from the superconformal point, which is captured by the BPS spectrum of the massive theory. The generalization of this idea to the $4 \mathrm{~d} \mathcal{N}=2$ theory, was the motivation of [6] which we next turn to.

\section{$3 \quad$ BPS monodromy and $4 \mathrm{~d}$ index}

We now move on to the $4 \mathrm{~d} \mathcal{N}=2$ supersymmetric theories. Here again we wish to connect the superconformal index computation to some computation when we move away from the conformal point, i.e. as we move on to the Coulomb branch. Just as in $2 d$ there are BPS states, which undergo wall crossing, and we wish to connect some invariant data on both sides.

Let us first start with the superconformal side. The superconformal index is defined as $[11,12]$ (see appendix B for more detail)

$$
\mathcal{I}(p, q, t)=\operatorname{Tr}(-1)^{F} p^{J_{34}+r} q^{J_{12}+r} t^{R-r} e^{-\beta H},
$$


where $J_{12}, J_{34}$ are the Cartan generators of the $\mathrm{SO}(4) \subset \mathrm{SO}(4,2)$ conformal group, $R$ is the Cartan generator of the $\mathrm{SU}(2)_{R}$ symmetry of $\mathcal{N}=2$ theories and $r$ is the $\mathrm{U}(1)_{r}$ symmetry of the conformal theory. The index can be viewed as the partition function of the conformal theory on $S^{1} \times S^{3}$ where as we go around $S^{1}$ we rotate the $S^{3}$ and we have turned on specific fugacities for the $R, r$ symmetries. The Hamiltonian $H$ is a $Q$ commutator, where $Q$ is a supercharge commuting with all operators inserted in the trace (3.1), and so the partition function does not depend on $\beta$ and for this reason we sometimes omit writing the $e^{-\beta H}$ insertion.

It is important to understand better this geometry. For this it is more convenient to view $S^{1} \times S^{3}$ as a complex manifold, known as the Hopf surface, with complex moduli parameterized by $(p, q)$ which we now describe.

\subsection{The Hopf surface}

Consider the space

$$
W=(\mathbb{C} \times \mathbb{C}-\{0,0\}) / \mathbb{Z},
$$

where the generator of $\mathbb{Z}$ acts as

$$
\left(z_{1}, z_{2}\right) \rightarrow\left(q z_{1}, p z_{2}\right)
$$

with $0<|q|,|p|<1$. $W$ is a complex manifold which is topologically $S^{3} \times S^{1}$. To see this, view $S^{3}$ as the loci in the complex 2-plane where $1=\left|w_{1}\right|^{2}+\left|w_{2}\right|^{2}$. Now consider the map

$$
f: S^{3} \times \mathbb{R} \rightarrow \mathbb{C} \times \mathbb{C}-\{0,0\},
$$

defined by $\left\{\left(w_{1}, w_{2}\right), t\right\} \mapsto\left(z_{1}, z_{2}\right)=\left(q^{t} w_{1}, p^{t} w_{2}\right)$. This map is a bijection. To see this note that given any $\left(z_{1}, z_{2}\right)$ there is a unique $\left\{\left(w_{1}, w_{2}\right), r\right\}$ which maps to it, i.e. the unique ${ }^{2} t$ such that $\left|z_{1} / q^{t}\right|^{2}+\left|z_{2} / p^{t}\right|^{2}=1$. To get $W$, note that modding out by $\mathbb{Z}$ simply identifies $t \sim t+1$, and thus $W$ has the topology of $S^{3} \times \mathbb{R} / \mathbb{Z} \equiv S^{3} \times S^{1}$.

The Hopf surface $W$ contains two natural tori: consider $z_{1}=0$ (which corresponds also to $\left.w_{1}=0\right)$. Over this point the manifold is given by $z_{2} \sim p z_{2}$ which defines a torus with complex structure given by $p$. Similarly over the point $z_{2}=0$ (which corresponds to $w_{2}=0$ ) we get $z_{1} \sim q z_{1}$ which defines a torus with complex structure $q$.

Note that if you delete the circle given by $w_{2}=0$ (i.e. the circle $\left|w_{1}\right|^{2}=1$ ) from $S^{3}$, you get a non-compact space which has the topology of

$$
\frac{1}{2} S^{3} \times S^{1}
$$

where $\frac{1}{2} S^{3}$ has the topology of a solid torus: $\mathbb{C} \times S^{1}$.

\footnotetext{
${ }^{2}$ To see that such $t$ is unique, note that $\left|z_{1} / q^{t}\right|^{2}+\left|z_{2} / p^{t}\right|$ is a monotonic function of $t$ which varies in the range $(0, \infty)$ for $t \in \mathbb{R}$.
} 


\subsection{A specialization of the $4 \mathrm{~d}$ index}

We now consider the specialization of the $4 \mathrm{~d}$ index by setting $t=q p^{N+1}$. This leads to

$$
\mathcal{I}^{\prime}(p, q)=\operatorname{Tr}(-1)^{F} p^{N(R-r)} p^{J_{34}+R} q^{J_{12}+R} .
$$

There are two special cases of this specialization: if $N=0$, this partition function is the same as the partition function on the Hopf surface of the $\mathcal{N}=2$ theory with Witten's topological twist [13], because in the topologically twisted theory the $\mathrm{SO}(4)$ generators are $J_{12}^{\prime}=J_{12}+R$ and $J_{34}^{\prime}=J_{34}+R$. In this case the partition function should not depend on the metric nor the complex structure of the manifold, and so the partition function (3.6) with $N=0$ does not depend on $p, q$. For $N=-1$ we get the Schur limit of the index studied in $[14,15]$ which is expected to give a reduction of the index to the partition function of a $2 \mathrm{~d}$ chiral algebra [8]. In that limit the index will not depend on $p$ but will still depend on $q$ and is characterized by a chiral algebra. For other values of $N$ we have an object which a priori depends on both $p, q$. It can be interpreted as the topologically twisted theory on $S^{1} \times S^{3}$ where in addition as we go around the $S^{1}$ we mod out by the action of $p^{N(R-r)}$. In other words, in addition to the usual twist we have introduced a chemical potential for the $(R-r)$ charge. This is very similar to the $2 \mathrm{~d}$ case where we inserted $z^{J_{0}}$. Here $R-r$ plays the role of $J_{0}$. Just as in the $2 \mathrm{~d}$ case we need a further specialization to connect to BPS spectra: thus we further specialize $p \rightarrow e^{2 \pi i}$, i.e. we define the index

$$
\mathcal{I}_{N}(q)=\operatorname{Tr}(-1)^{F} e^{2 \pi i N(R-r)} e^{2 \pi i\left(J_{34}+R\right)} q^{J_{12}+R} .
$$

Note that this specialization can only be realized as a limiting instance of the Hopf surface. In particular the torus over $w_{1}=0$ degenerates. Over $w_{1}=0$ there are two circles which form a torus: one is the circle $w_{2} \rightarrow w_{2} p^{t+\phi}$. The other is $w_{2} \rightarrow w_{2} e^{i \theta}$. In the limit $p \rightarrow e^{2 \pi i}$ the size of the circle corresponding to phase of $w_{2}$ is much bigger than that of the circle $t \rightarrow t+\phi$. If we wish to keep the size of $t$-circle finite, we effectively make $w_{2}$ infinitely large, and so the corresponding circle cannot shrink. This effectively deletes the point $w_{2}=0$ where the corresponding circle would have shrunk from the geometry. Thus, we get an $\frac{1}{2} S^{3} \times S^{1}$ geometry in this limiting case. View the $\frac{1}{2} S^{3}$ as $\left(w_{1}, \frac{w_{2}}{\left|w_{2}\right|}\right)=\mathbb{C} \times S^{1}$. As we go around $S^{1}$ we mod out this geometry according to

$$
t \rightarrow t+1: \quad\left(w_{1}, \frac{w_{2}}{\left|w_{2}\right|}\right) \rightarrow\left(q w_{1}, \frac{w_{2}}{\left|w_{2}\right|}\right) .
$$

In other words we have a Melvin cigar geometry. This is the geometric part of the interpretation of the $\mathcal{I}_{N}(q)$. This is also accompanied with the Witten twist due to the $R$ action. But now the action is not purely topological because, as we go around the circle, we have in addition the action of the operator $e^{2 \pi i N(r-R)}$. In other words we have a geometry of the form

$$
W^{\prime}=S_{q, N(R-r)}^{1} \times S^{1} \times \mathbb{C},
$$

where the notation $S_{q, N(R-r)}^{1}$ means that as we go around that $S^{1}$ we both rotate $\mathbb{C}$ by $q$ and has a fugacity action by $e^{2 \pi i N(R-r)}$. 


\subsection{BPS mondromy and the specialized index}

If we consider a deformation of a $\mathcal{N}=2 \mathrm{SCFT}$ in $d=4$ to its Coulomb branch, we find a tower of BPS states. This tower of BPS states can change as we move in the Coulomb branch as we cross walls of marginal stability [2,3], as was shown in [5] (and its refinement in $[16,17])$. If one consider an ordered product of BPS states, ordered by their central charge phases, where each BPS particle of helicity $s$ and charge $\gamma$ contributes the factor

$$
\mathcal{Z}_{\gamma}=\prod_{n=0}^{\infty}\left(1-q^{n+\frac{1}{2}+s} X_{\gamma}\right)^{(-1)^{2 s}}
$$

to the monodromy operator

$$
M(q)=T\left(\prod_{\gamma} \mathcal{Z}_{\gamma}\right)
$$

Here $X_{\gamma}$ form a quantum torus algebra $\mathbb{T}$ :

$$
X_{\gamma} X_{\gamma^{\prime}}=q^{\left\langle\gamma, \gamma^{\prime}\right\rangle} X_{\gamma^{\prime}} X_{\gamma} \quad \gamma, \gamma^{\prime} \in \Gamma
$$

and $\left\langle\gamma, \gamma^{\prime}\right\rangle \in \mathbb{Z}$ is the electro-magnetic symplectic pairing on the lattice $\Gamma$ of conserved charges. $M(q)$ is a wall-crossing invariant up to conjugation. There is another version of $M(q)$, closely related to the above operator, which is also invariant [6]: one replaces the compact quantum dilogarithm (3.10) with its non-compact version

$$
\mathcal{Z}_{\gamma}^{\mathrm{nc}}=\mathcal{Z}_{\gamma}\left(q, X_{\gamma}\right) / \mathcal{Z}_{\gamma}\left(\hat{q}, \hat{X}_{\gamma}\right)
$$

where now $X_{\gamma}$ and $\hat{X}_{\gamma}$ form a dual pair of quantum torus algebras

$$
X_{\gamma} X_{\gamma^{\prime}}=q^{\left\langle\gamma, \gamma^{\prime}\right\rangle} X_{\gamma^{\prime}} X_{\gamma} \quad X_{\gamma} \hat{X}_{\gamma^{\prime}}=\hat{X}_{\gamma^{\prime}} X_{\gamma}, \quad \hat{X}_{\gamma} \hat{X}_{\gamma^{\prime}}=\hat{q}^{-\left\langle\gamma, \gamma^{\prime}\right\rangle} \hat{X}_{\gamma^{\prime}} \hat{X}_{\gamma},
$$

related as follows

$$
\begin{array}{ll}
q=e^{2 \pi i \tau}, & X_{\gamma}=e^{i x_{\gamma}}, \\
\hat{q}=e^{-2 \pi i / \tau}, & \hat{X}_{\gamma}=e^{i x_{\gamma} / \tau} \quad \text { with } \quad\left[x_{\gamma}, x_{\gamma^{\prime}}\right]=-2 \pi i \tau\left\langle\gamma, \gamma^{\prime}\right\rangle .
\end{array}
$$

As one can see this is very similar to the $2 \mathrm{~d}$ story. The main difference is that now the monodromy operator depends on a parameter $q$. In the above definition of $M(q)$ it would be natural to include also the massless BPS state, namely the photons of the Coulomb branch. They would correspond to $\gamma=0$ and $s= \pm 1 / 2$ leading to $\eta^{-2 r}(q)$ additional factor to $M(q)$. More precisely we need to delete a zero mode (corresponding to $n=0, s=-1 / 2)$ to get that. This will be important to keep in mind. We shall write $\mathcal{M}(q)$ for the monodromy $M(q)$ dressed by the massless photon factor.

An explanation of this result was provided in [6] as follows: consider an $\mathcal{N}=2$ theory in 4 dimensions. Compactify it on the Melvin cigar which is the geometry $S_{q}^{1} \times \mathbb{C}$ where as you go around $S^{1}$ you rotate $\mathbb{C}$ by multiplying it by $q$. One obtains a theory in $1 \mathrm{~d}$ with 4 supercharges. In terms of supersymmetric quantum mechanics (SQM) data, this theory has infinitely many vacua and the analog of the finite dimensional matrix contribution to the monodromy operator [1], gets promoted to the operator $\mathcal{Z}_{\gamma}$ given in (3.10). One can view the 
parameter $q$ as a regularization of the computation. ${ }^{3}$ Thus consider the following geometry:

$$
S_{N(R-r)}^{1} \times S_{q}^{1} \times \mathbb{C}
$$

This gets naturally interpreted as follows [6]: we compactify the theory from $4 \mathrm{~d}$ to $3 \mathrm{~d}$ on a circle where we turn on the global symmetry $g=\exp (2 \pi i N(r-R))$ as we go around the circle. Then we obtain a $3 \mathrm{~d}$ theory. If we consider the partition function of this $3 \mathrm{~d}$ theory on $\frac{1}{2} S^{3}$ this would give us the trace of the monodormy operator to the $N$-th power. To make the theory compact, and completing the $\frac{1}{2} S^{3}$ to $S^{3}$, we proceed in two different ways. One way is simply to consider the squashed partition function on $S^{3}$ [19] where the R-twisting needed to define it is inherited from the $4 \mathrm{~d} \mathrm{SU}(2)_{R}$ symmetry (rather than the one natural from 3d SCFT perspective). This gets identified with the trace of the non-compact version of the monodromy operator $M(q)^{\text {nc }}$ made of non-compact quantum dilogs. This was already suggested in [6] and will not be the main focus of this paper. ${ }^{4}$

However to connect to the index it is best to modify this construction slightly and compactify to $S^{3}$ differently: we first compactify the theory on $\mathbb{C}$ which is the cigar geometry with the usual topological twisting. This leads to a $2 \mathrm{~d}$ theory with $(2,2)$ supersymmetry due to the non-trivial cigar geometry. This geometry has an extra U(1) symmetry involving rotation of $\mathbb{C}$. In this reduced theory we can consider the $2 \mathrm{~d}$ BPS monodromy, but keep track also of the extra U(1) symmetry of the BPS solitons by weighting them with $q^{\text {charge }}$. This will lead again to the $\mathcal{Z}_{\gamma}$ factors in eq. (3.10). In other words, we consider instead

$$
S_{q, N(R-r)}^{1} \times S^{1} \times \mathbb{C}
$$

but this is precisely the geometry $W^{\prime}$ that our limiting case of the index computes. We thus conclude

$$
\operatorname{Tr} \mathcal{M}(q)^{N}=Z\left(S_{q, N(R-r)}^{1} \times S^{1} \times \mathbb{C}\right)=\mathcal{I}_{N}(q)=\left.\mathcal{I}(p, q, t)\right|_{t \rightarrow q p^{N+1}, p \rightarrow e^{2 \pi i}} .
$$

This is essentially the connection anticipated in [7] with a minor modification. The case $N=-1$, the Schur case, is a special case of this which was already conjectured and checked in some examples in [9].

\section{Lagrangian theories}

The $4 \mathrm{~d} \mathcal{N}=2$ superconformal index is a refined Witten index on $S^{3} \times S^{1}$ evaluated by a trace formula [11, 12],

$$
\mathcal{I}(p, q, t)=\operatorname{Tr}(-1)^{F} p^{j_{1}-j_{2}+r} q^{j_{1}+j_{2}+r} t^{R-r} e^{-\beta \delta_{1-}}, \quad \delta_{1-}=2\left\{\mathcal{Q}_{1-}, \mathcal{Q}_{1-}^{\dagger}\right\},
$$

\footnotetext{
${ }^{3}$ For a more detailed discussion of the role of $q$ in this context, see [18].

${ }^{4}$ This is nevertheless an interesting construction because the $\left(M(q)^{\mathrm{nc}}\right)^{k}$ is strictly the identity operator if the $r$ charges are multiples of $1 / k$ and so by studying this operator we can deduce what fractions appear in the R-charges. Therefore this will only lead to inequivalent result for $N \bmod k$. In particular for all the Lagrangian theories where $k=1$ there is nothing interesting to compute in this version. This is similar to the case of $2 \mathrm{~d}$ for $(2,2)$ supersymmetric gauged linear sigma models where the R-charges are integer (or half-integer).
} 
where $\mathcal{Q}_{1-}$ is one of the eight supercharges in $4 \mathrm{~d} \mathcal{N}=2$ superconformal algebra (SCA), $j_{1}, j_{2}, R$, and $r$ label the $\mathrm{SU}(2)_{1} \times \mathrm{SU}(2)_{2} \times \mathrm{SU}(2)_{R} \times \mathrm{U}(1)_{r}$ symmetry of $4 \mathrm{~d} \mathcal{N}=2$ SCA. Note that $J_{12}=j_{1}+j_{2}, J_{34}=j_{1}-j_{2}$. By definition the index (4.1) gets contribution from the states annihilated by $\mathcal{Q}_{1-}$, or equivalently, states satisfying

$$
\delta_{1-}=\Delta-2 j_{1}-2 R-r=0 .
$$

We can further twist the index with fugacities $\mu_{i}$ dual to the Cartan generators $F_{i}$ of the flavor symmetry $\mathcal{F}$,

$$
\mathcal{I}\left(p, q, t, \mu_{i}\right)=\operatorname{Tr}(-1)^{F} p^{j_{1}-j_{2}+r} q^{j_{1}+j_{2}+r} t^{R-r} \prod_{i} \mu_{i}^{F_{i}} e^{-\beta \delta_{1-}} .
$$

The superconformal index is invariant under exactly marginal deformations. Therefore for Lagrangian theories we can always compute the exact index in the free theory limit. For a Lagrangian theory with gauge group $\mathcal{G}$ and matter in the representation $\oplus_{i}\left(\mathcal{R}_{i} \otimes \mathcal{R}_{i}^{F}\right)$ of gauge group $\mathcal{G}$ and flavor group $\mathcal{F}$, the index is given by

$$
\mathcal{I}(p, q, t)=\int[d \mathcal{G}] \mathcal{I}^{V}(p, q, t ; \mathcal{G}) \prod_{i} \mathcal{I}^{H}\left(p, q, t ; \mathcal{R}_{i} \otimes \mathcal{R}_{i}^{F}\right)
$$

$[d \mathcal{G}]$ being the Haar measure of the gauge group $\mathcal{G}, \mathcal{I}^{H}$ and $\mathcal{I}^{V}$ the indices of $\mathcal{N}=2$ hypermultiplets and vector-multiplets respectively. They are given by

$$
\begin{aligned}
\mathcal{I}^{H}\left(p, q, t ; \mathcal{R} \otimes \mathcal{R}^{F}\right) & =\prod_{w \in \mathcal{R}} \prod_{v \in \mathcal{R}^{F}} \prod_{m, n=0}^{\infty} \frac{\left(1-z^{w} \mu^{v} t^{-\frac{1}{2}} p^{m+1} q^{n+1}\right)}{\left(1-z^{w} \mu^{v} t^{\frac{1}{2}} p^{m} q^{n}\right)} \\
\mathcal{I}^{V}(p, q, t ; \mathcal{G}) & =\prod_{\alpha \in \Delta(\mathcal{G})} \prod_{m=0}^{\infty}\left(1-z^{\alpha} p^{m+1}\right)\left(1-z^{\alpha} q^{m+1}\right) \prod_{m, n=0}^{\infty} \frac{1-t p^{m} q^{n} z^{\alpha}}{1-t^{-1} p^{m+1} q^{n+1} z^{\alpha}} .
\end{aligned}
$$

Here we introduced the flavor fugacity $\mu$ and gauge fugacity $z$. In equation (4.5) $w \in \mathcal{R}$ (resp. $v \in \mathcal{R}^{F}$ ) are the weights of the gauge (flavor) representation $\mathcal{R}$ (resp. $\mathcal{R}^{F}$ ), and $\Delta(\mathcal{G}$ ) is the set of all roots of the gauge group $\mathcal{G}$ (the zero root being counted with multiplicity $r=\operatorname{rank} \mathcal{G}) . z^{\alpha}$ is a short-hand notation for $z^{\alpha} \equiv \prod_{i=1}^{r} z_{i}^{\alpha_{i}}$. For example, for $\mathcal{G}=\mathrm{SU}(2)$, $z^{\alpha}$ takes the values $z^{2}, 1, z^{-2}$.

The physical meaning of equation (4.4) is now clear. $\mathcal{I}^{V}$ and $\mathcal{I}^{H}$ are partition functions of supersymmetric states of the respective multiplets. The integrand of (4.4) is the partition function of all supersymmetric states of the given theory at zero coupling, and $\int[d \mathcal{G}]$ projects onto the gauge singlet part of the supersymmetric spectrum.

\subsection{Specialized index for the Lagrangian theory}

As discussed in previous section, to relate the superconformal index to chiral algebra we need to take a specialization of the index by setting $t=q p^{N+1}$. From the equation (4.3), the specialized index is

$$
\mathcal{I}_{N}\left(p, q ; \mu_{i}\right)=\operatorname{Tr}(-1)^{F} p^{j_{1}-j_{2}+R+N(R-r)} q^{j_{1}+j_{2}+R} \prod_{i} \mu_{i}^{F_{i}} e^{-\beta \delta_{1-}} .
$$


Since the indices of Lagrangian theories are built upon indices of free hypermultiplets and vector multiplets, it is enough to discuss these two cases.

When $N=-1$ the specialized index is the Schur index $[14,15]$ and the dependence on $p$ drops off automatically.

\subsubsection{Insertion of Wilson loop line operators}

Insertion of half-BPS operators in Schur index was discussed by [20, 21]. The line operators discussed there preserve the supercharge $\mathcal{Q}_{1-}+\tilde{\mathcal{Q}}_{1-}$ and commute with $J_{12}+R$. These line operators cannot be inserted in the index for arbitrary values of $p, q \cdot{ }^{5}$ However something remarkable happens precisely for the specializations we are interested in. Even though $\mathcal{Q}_{1-}$ has $J_{34}+R+N(R-r)$ charge 0 , the corresponding charge of $\tilde{\mathcal{Q}}_{1-}$ is $N+1$, and the operator $J_{34}+R+N(R-r)$ will not commute with the supercharge $\mathcal{Q}_{1-}+\tilde{\mathcal{Q}}_{1-}$ used to define the index in the presence of line operators (except for $N=-1$ ). Nevertheless $\mathcal{Q}_{1-}+\tilde{\mathcal{Q}}_{1-}$ still commutes with the discrete symmetry

$$
e^{2 \pi i\left(J_{34}+R+N(R-r)\right)}
$$

which is the specialization of interest to us, $p \rightarrow \exp (2 \pi i)$. Thus our index specializations could have been pointed out a priori as the class of superconformal indices consistent with the insertion of supersymmetric line operators.

For Lagrangian theories, the specialized index with insertion of Wilson line operators in representation $R$ and $\bar{R}$ wrapping $S^{1}$ and placed at antipodal points of $S^{3}$ is

$$
\mathcal{I}_{N}(q)=\int[d \mathcal{G}] \chi_{R}(q) \chi_{\bar{R}}(q) \mathcal{I}_{N}^{V}(q ; \mathcal{G}) \prod_{i} \mathcal{I}_{N}^{H}\left(q ; \mathcal{R}_{i} \otimes \mathcal{R}_{i}^{F}\right)
$$

with $\mathcal{I}_{N}^{H}$ and $\mathcal{I}_{N}^{V}$ the specialized indices for hyper and vector multiplets which will be discussed in detail below, and $\chi_{R}(q)\left(\chi_{\bar{R}}(q)\right)$ the character of the representation $R(\bar{R})$ which contributed from Wilson line operators. The other line operators which play an important role later are

$$
\operatorname{det}(1-p U)_{\mathrm{Adj}}^{ \pm}
$$

with $U$ the holonomy of gauge group along $S^{1}$. For gauge group $\mathrm{SU}(k)$ the operator (4.8) can be understood as a combination of Wilson line operators in fundamental and antifundamental representations. One can also similarly consider insertion of 't Hooft and dyonic line operators, which we will not spell out here as we will not need them for the present paper.

\subsubsection{Free hypermultiplets}

The $4 \mathrm{~d}$ index of a free (full) hypermultiplet is given by

$$
\mathcal{I}^{H}=\prod_{i, j=0}^{\infty} \frac{\left(1-t^{-\frac{1}{2}} q^{i+1} p^{j+1} u\right)}{\left(1-t^{\frac{1}{2}} q^{i} p^{j} u\right)} \frac{\left(1-t^{-\frac{1}{2}} q^{i+1} p^{j+1} u^{-1}\right)}{\left(1-t^{\frac{1}{2}} q^{i} p^{j} u^{-1}\right)}
$$

\footnotetext{
${ }^{5}$ We would like to thank Kimyeong Lee for a discussion on this point.
} 
with $u$ the fugacity of $\mathrm{U}(1)$ flavor symmetry of a free hypermultiplet. After setting $t=$ $q p^{N+1}$ we have

$$
\mathcal{I}_{N}^{H}= \begin{cases}\prod_{i=0}^{|N|-1} \prod_{m=0}^{\infty} \frac{1}{\left(1-q^{m+\frac{1}{2}} p^{\frac{N+1}{2}+i} u\right)} \frac{1}{\left(1-q^{m+\frac{1}{2}} p^{\frac{N+1}{2}+i} u^{-1}\right)} & \text { for } N<0 \\ \prod_{i=0}^{N-1} \prod_{m=0}^{\infty}\left(1-q^{m+\frac{1}{2}} p^{\frac{1}{2}-\frac{N}{2}+i} u\right)\left(1-q^{m+\frac{1}{2}} p^{\frac{1}{2}-\frac{N}{2}+i} u^{-1}\right) & \text { for } N>0\end{cases}
$$

We can write this in terms of a theta function as

$$
\mathcal{I}_{N}^{H}=(q)_{\infty}^{-N} \prod_{-\frac{|N|-1}{2} \leq j \leq \frac{|N|-1}{2}} \theta\left(q^{\frac{1}{2}} p^{j} u ; q\right)^{\operatorname{sign}(N)} \stackrel{p \rightarrow 1}{\longrightarrow}(q)_{\infty}^{-N} \theta\left(q^{\frac{1}{2}} u ; q\right)^{N}
$$

where

$$
\theta(z ; q)=\prod_{i \geq 0}\left(1-q^{i}\right)\left(1-z q^{i}\right)\left(1-z^{-1} q^{i+1}\right) \equiv(q ; q)_{\infty}(z ; q)_{\infty}(q / z ; q)_{\infty}
$$

and we used the standard short-hand notations

$$
(a)_{\infty} \equiv(a ; q)_{\infty}:=\prod_{k=0}^{\infty}\left(1-a q^{k}\right) .
$$

In particular, (4.11) yields the Schur index for $N=-1$. We see that the specialized index $\mathcal{I}_{N}^{H}$ looks like $N$ copies of the same object (a negative number of copies meaning, as always, $|N|$ copies with the opposite Fermi/Bose statistics).

To understand the specialized index from the viewpoint of operator counting, we can check in table 8 of appendix B the indices of single letters contributing to the index. When $N<0$, we find that letters contributing to the specialized index of hypers are

$$
\partial_{z}^{i} \partial_{w}^{j} q, \quad \partial_{z}^{i} \partial_{w}^{j} \tilde{q}, \quad \text { with } i \in \mathbb{Z}_{\geq 0}, j=0,1, \cdots,|N|-1,
$$

where we use the notation $\partial_{z} \equiv \partial_{+\dot{+}}$ and $\partial_{w} \equiv \partial_{+\dot{-}}$. The derivatives $\partial_{z}$ and $\partial_{w}$ contribute to the index by $q$ and $p$ respectively. When $N>0$, the letters contributing to the specialized index are

$$
\partial_{z}^{i} \partial_{w}^{j} \psi_{+}, \quad \partial_{z}^{i} \partial_{w}^{j} \tilde{\psi}_{+}, \quad \text { with } i \in \mathbb{Z}_{\geq 0}, j=0,1, \cdots, N-1 .
$$

In other words, the specialized index counts the operator $q, \tilde{q}$ or $\psi_{+}, \tilde{\psi}_{+}$with arbitrary number of derivatives $\partial_{+\dot{+}}$ and up to $|N|-1$ derivatives $\partial_{+\dot{-}}$.

It is clear from the equation (4.10) that the specialized index of a free hypermultiplet $\mathcal{I}_{N}^{H}$ is the same as the partition function of $N 2 \mathrm{~d}$ complex bosons with spin $\frac{1}{2}$ for $N<0$. When $N>0$, the specialized index describes $N 2 \mathrm{~d}$ complex fermions with the correct spin $1 / 2$. In both cases, $p$ becomes a fugacity for the $\mathrm{U}(1)_{p}$ charge corresponding to the angular momentum in the $w$-direction. This shows that for the specialization of the index at $t=q p^{N+1}$ there corresponds a $2 \mathrm{~d}$ chiral algebra $\mathcal{A}_{N}$, at least for the free theory. This generalizes the $2 \mathrm{~d}$ chiral algebra associated to the $4 \mathrm{~d} \mathcal{N}=2$ theories discussed in Beem 
et al. [8]. In our language, their case corresponds to picking $N=-1$ among all possible choices of $N$. The relation between the specialized index $\mathcal{I}_{N}$ and the $2 \mathrm{~d}$ chiral algebra $\mathcal{A}_{N}$ will be discussed further in section 4.2 .

It is not difficult to write down the specialized index for hypermultiplets transforming in the representation $\mathcal{R}$,

$$
\mathcal{I}_{N}^{H}(\mathcal{R})= \begin{cases}\prod_{w \in \mathcal{R}} \prod_{i=0}^{|N|-1} \prod_{m=0}^{\infty} \frac{1}{\left(1-q^{m+\frac{1}{2}} p^{\frac{N+1}{2}+i} z^{w}\right)} \frac{1}{\left(1-q^{m+\frac{1}{2}} p^{\frac{N+1}{2}+i} z^{-w}\right)} \quad \text { for } N<0, \\ \prod_{w \in \mathcal{R}} \prod_{i=0}^{N-1} \prod_{m=0}^{\infty}\left(1-q^{m+\frac{1}{2}} p^{\frac{1}{2}-\frac{N}{2}+i} z^{w}\right)\left(1-q^{m+\frac{1}{2}} p^{\frac{1}{2}-\frac{N}{2}+i} z^{-w}\right) & \text { for } N>0 .\end{cases}
$$

This gives the partition function of $|N|$ copies of $2 d$ complex spin- $\frac{1}{2}$ bosons/fermions in representation $\mathcal{R}$.

\subsubsection{Free vector multiplets}

Now, let us consider the case of free vector multiplets. As we will see later, we encounter subtle new features involving the nature of zero modes. The index of a free vector multiplet is given by

$$
\mathcal{I}^{V}=\prod_{i=0}^{\infty}\left(1-p^{i+1}\right)\left(1-q^{i+1}\right) \prod_{i, j=0}^{\infty} \frac{1-t p^{i} q^{j}}{1-t^{-1} p^{i+1} q^{j+1}} .
$$

After specialization to $t=q p^{N+1}$, it becomes

$$
\mathcal{I}_{N}^{V}=\prod_{i=0}^{\infty}\left(1-p^{i+1}\right)\left(1-q^{i+1}\right) \prod_{i, j=0}^{\infty} \frac{1-p^{i+N+1} q^{j+1}}{1-p^{i-N} q^{j}} .
$$

For $N \geq 0$ the denominator vanishes and the specialized index diverges. Let us first consider the simpler case $N<0$.

Negative $\boldsymbol{N}$. When $N<0$, the specialized index is

$$
\mathcal{I}_{N}^{V}=\prod_{i=1}^{|N|-1}\left(1-p^{i}\right) \prod_{i=0}^{|N|-1} \prod_{j=0}^{\infty}\left(1-p^{i} q^{j+1}\right)\left(1-p^{-i} q^{j+1}\right) .
$$

It reduces to the Schur index at $N=-1$. The 4 d letters contributing to the specialized index of vector multiplets are

$$
\begin{array}{lll}
\partial_{z}^{j} \partial_{w}^{|N|-1} \lambda_{1+}, & \partial_{z}^{j} \bar{\lambda}_{1 \dot{-}}, & \text { with } j \in \mathbb{Z}_{\geq 0}, \\
\partial_{z}^{j} \partial_{w}^{i-1} \lambda_{1+}, & \partial_{z}^{j} \partial_{w}^{i-1} \bar{\lambda}_{1 \dot{+}}, & \text { with } j \in \mathbb{Z}_{\geq 0}, \quad i=1,2, \cdots,|N|-1 .
\end{array}
$$

Through the state-operator correspondence, the specialized index is a partition function over the corresponding states on $S^{3}$. The prefactor $\prod_{i=1}^{|N|-1}\left(1-p^{i}\right)$ represents the contribution of the zero modes $\partial_{w}^{i} \bar{\lambda}_{1+}(i=0,2, \cdots,|N|-2)$. As in the hypermultiplet case, arbitrary number of derivatives $\partial_{z}$ contribute to the index while $\partial_{w}$ contribute to the specialized index up to some finite number. The specialized index can also be interpreted as 
the partition function of $|N|$ copies of $b c$-system $b^{(i)}$ and $c^{(i)}(i=0,1, \cdots,|N|-1)$ with $\mathrm{U}(1)_{p}$ charges $\pm i$.

For a vector multiplet of the non-Abelian gauge group $\mathcal{G}$ of rank $r$ at zero coupling constant, the specialized index at $N<0$ is

$$
\mathcal{I}_{n}^{V}(\mathcal{G})=\prod_{\alpha \in \Delta(\mathcal{G})} \prod_{i=1}^{|N|-1}\left(1-z^{\alpha} p^{i}\right) \prod_{i=0}^{|N|-1} \prod_{j=0}^{\infty}\left(1-z^{\alpha} p^{i} q^{j+1}\right)\left(1-z^{\alpha} p^{-i} q^{j+1}\right)
$$

where, as before, $\Delta(\mathcal{G})$ is the set of all roots of $\mathcal{G}$ the zero root counted with multiplicity $r$.

The index has a zero at $p \rightarrow e^{2 \pi i}$ of order $r(|N|-1)$, because in this limit the $r$ Cartan elements of $\partial_{w}^{i} \bar{\lambda}_{1+}$ behave as $r(|N|-1)$ fermionic zero modes. As we normally do in $2 \mathrm{~d}$, to avoid the problem, we trace over the states with all fermionic zero modes excited. In other words, to get a non-zero answer, we have to slightly modify the index computation by inserting a suitable operator. Of course we need to make sure the operator we insert is gauge-invariant. One choice we can make is the operator

$$
\mathcal{O}(z)=\operatorname{det}_{\text {Adj }}\left(\prod_{i=1}^{|N|-1} \partial_{w}^{i-1} \bar{\lambda}_{1 \dot{+}}(z, 0)\right)
$$

and consider trace over the vacuum with the above operator inserted,

$$
\mathcal{O}(0)|0\rangle
$$

defined by taking the trace over the corresponding space of states. This removes the whole prefactor $\prod_{\alpha \in \Delta(\mathcal{G})} \prod_{i=1}^{|N|-1}\left(1-z^{\alpha} p^{i}\right)$ in the equation (4.21), and we get

$$
\mathcal{I}_{N}^{V \text {,finite }}(\mathcal{G})=\prod_{\alpha \in \Delta(\mathcal{G})} \prod_{i=0}^{|N|-1} \prod_{j=0}^{\infty}\left(1-z^{\alpha} p^{i} q^{j+1}\right)\left(1-z^{\alpha} p^{-i} q^{j+1}\right) .
$$

This is the partition function of $|N|-1$ copies of the $b c$-system transforming in the adjoint of the gauge group $\mathcal{G}$.

Instead of inserting a point-like operator we can also use line operators to absorb the fermionic zero modes: notice that the insertion of $\mathcal{O}$ is equivalent to computing the specialized index with an insertion of the form

$$
\prod_{i=1}^{|N|-1} \frac{1}{\operatorname{det}_{\mathrm{Adj}}\left(1-p^{i-1} U\right)}
$$

We can expand this insertion in characters of $\mathcal{G}$ as $\sum_{R} a_{R}(p) \operatorname{Tr}_{R} U$, so that (4.25) can be thought of as a generating function for certain line operators. The line operator index has been studied in [20,21]. In order to preserve supersymmetry, the line operators should wrap the time circle $S^{1}$ and be placed at the north and south pole of the $S^{3}$. Therefore, all the line operator indices should involve insertions of the form $\operatorname{Tr}_{R \otimes \bar{R}} U$ in the integral. In our case, the representations which appear are the tensor powers of the adjoint representation. For example, for $\mathrm{SU}(k)$ gauge group we can simply use the fact that $k \otimes \bar{k}=\operatorname{adj} \oplus 1$ to construct line operators which effectively insert the operator (4.25) in the index computation. 
Positive $\boldsymbol{N}$. When $N \geq 0$, there is a divergence coming from the term $1-t^{-1} p^{N+1} q$ in the denominator of the equation (4.17). This term comes from the contribution of $\partial_{w}^{N} \bar{\phi}$. Formally, the specialized index of a U(1) vector multiplet can be written as,

$$
\mathcal{I}_{N}^{V}=\prod_{i=0}^{N} \frac{1}{1-p^{-i}} \prod_{i=1}^{N} \prod_{j=0}^{\infty} \frac{1}{\left(1-p^{-i} q^{j+1}\right)\left(1-p^{i} q^{j+1}\right)} .
$$

The $4 \mathrm{~d}$ letters contributing to the index are as follows:

$$
\begin{gathered}
\partial_{w}^{i} \bar{\phi}, \quad \text { with } i=0,1,2, \cdots, N \\
\partial_{z}^{j+1} \partial_{w}^{i} \bar{\phi}, \quad \partial_{z}^{j} \partial_{w}^{i} F_{++}, \quad \text { with } j=0,1, \cdots, \infty, \quad i=0,1, \cdots, N-1 .
\end{gathered}
$$

The prefactor $\prod_{i=0}^{N} \frac{1}{1-p^{-i}}$ originates from the contribution of $N+1$ zero modes $\partial_{w}^{i} \bar{\phi}$. Now we get the partition function of $N$ complex scalars with $\mathrm{U}(1)_{p}$ charges $\pm 1, \pm 2, \cdots, \pm N$ together with $N+1$ bosonic zero modes.

For the vector multiplet in non-Abelian gauge group $\mathcal{G}$ with zero coupling constant, formally the specialized index can be written as

$$
\mathcal{I}_{N}^{V}(\mathcal{G})=\prod_{\alpha \in \Delta(\mathcal{G})} \prod_{i=0}^{N} \frac{1}{1-z^{\alpha} p^{-i}} \prod_{i=1}^{N} \prod_{j=0}^{\infty} \frac{1}{\left(1-z^{\alpha} p^{-i} q^{j+1}\right)\left(1-z^{\alpha} p^{i} q^{j+1}\right)} \quad \text { for } N \geq 0 .
$$

Again there are $r$ copies of the divergent term $\prod_{i=0}^{N} \frac{1}{1-p^{-i}}$ from the Cartan part of $\mathcal{G}$ in the prefactor $\prod_{\alpha \in \Delta(\mathcal{G})} \prod_{i=0}^{N} \frac{1}{1-z^{\alpha} p^{-i}}$.

Under the limit $p \rightarrow e^{2 \pi i}$ we get extra poles because $\partial_{w}^{i} \bar{\phi}$ contains bosonic zero modes for each $i$. We remove them by taking the trace over the states with delta function inserted:

$$
\prod_{a \in \operatorname{Adj}}\left(\prod_{i=0}^{N} \delta\left(\partial_{w}^{i} \bar{\phi}^{a}(0)\right)\right)|0\rangle \text {. }
$$

We get

$$
\mathcal{I}_{N}^{V, \text { finite }}(\mathcal{G})=\prod_{\alpha \in \Delta(\mathcal{G})} \prod_{i=1}^{N} \prod_{j=0}^{\infty} \frac{1}{\left(1-z^{\alpha} p^{-i} q^{j+1}\right)\left(1-z^{\alpha} p^{i} q^{j+1}\right)} \quad \text { for } N \geq 0 .
$$

This can also be realized by insertion of line operators. It is equivalent to the specialized index with the line operator

$$
\prod_{i=0}^{|N|-1} \operatorname{det}\left(1-p^{i-1} U\right)_{\operatorname{Adj}}
$$

inserted.

\subsection{Chiral algebras}

In this section, we provide further evidence for the existence of sectors in the $4 \mathrm{~d} \mathcal{N}=2$ SCFT described by a chiral algebra. In particular we obtain a chiral algebra $\mathcal{A}_{N}$ labelled by an integer $N$ for the theory of free hypermultiplets and free vector multiplets. We also describe the chiral algebra for interacting theory in the limit $g \rightarrow 0$, by taking into account gauge invariance. We show that the partition function of the chiral algebra is given by the specialized superconformal index $\mathcal{I}_{N}(q)$ (with operator/line operator insertions). 


\subsubsection{Free theories}

We call the coordinates of the plane in the 12 direction $z$ and $\bar{z}$, and the coordinates of the plane in 34 direction $w$ and $\bar{w}$. In the discussion below, we are going to confine all the operators in the 12 plane $w=\bar{w}=0$.

Hypermultiplets. When $N<0$, we define the following operators $Q^{i}(z)$ and $\bar{Q}^{i}(z)$ on the $z$ plane,

$$
\begin{aligned}
Q^{(i)}(z) & \equiv \partial_{w}^{i} q(z, \bar{z}, 0,0)+\bar{z}(z \bar{z})^{|N|-1-i} \partial_{\bar{w}}^{|N|-1-i} \overline{\tilde{q}}(z, \bar{z}, 0,0), \\
\bar{Q}^{(i)}(z) & \equiv \partial_{w}^{|N|-1-i} \tilde{q}(z, \bar{z}, 0,0)-\bar{z}(z \bar{z})^{i} \partial_{\bar{w}}^{i} \bar{q}(z, \bar{z}, 0,0),
\end{aligned}
$$

where $i$ runs through $0,1, \cdots,|N|-1$. Note that $\bar{Q}(z)$ is not defined as the complex conjugate of $Q(z)$. The idea behind this construction is that only $q$ 's with limited number of derivatives on $w$ contribute to the specialized index and the specialized index is the same as the partition function of $|N|$ complex chiral bosons with wrong spin- $\frac{1}{2}$. At $N=-1$ there is no derivatives in $w$ direction and equation (4.32) reduces to chiral fields defined by equation (3.30) in Beem et al. [8] for a free hypermultiplet.

The $Q \bar{Q}$ operator product expansion (OPE) follows from the correlation function of $q(x)$,

$$
q(x) \bar{q}(0) \sim \frac{1}{|x|^{2}}+\cdots,
$$

with $|x|^{2}=z \bar{z}+w \bar{w}$. From this, we obtain

$$
Q^{(i)}(z) \bar{Q}^{(j)}(0)=-\bar{Q}^{(i)}(z) Q^{(j)}(0)=\frac{\delta^{i j}}{z}+\cdots,
$$

where the $\bar{z}$-dependence dropped out. The OPE is chiral in $z$ and the same as $|N|$ copies of $2 \mathrm{~d}$ symplectic scalars, which gives central charge $c^{2 d}=N$. Therefore, the chiral algebra for the free hypermultiplet is given by

$$
\mathcal{A}_{N}\left(\mathcal{T}_{H}\right)=\left\{\left\langle Q^{(i)}(z), \bar{Q}^{(i)}(z)\right\rangle|i=0, \cdots,| N \mid-1\right\} \quad \text { for } N<0 .
$$

We indeed reproduce the partition function from the specialized index $\mathcal{I}_{N}^{H}=$ $(q)_{\infty}^{|N|} \theta\left(q^{\frac{1}{2}} u ; q\right)^{-|N|}$.

When $N>0$ the we define the corresponding chiral fields as

$$
\begin{aligned}
& \Psi^{(i)}(z) \equiv \partial_{w}^{i} \psi_{+}(z, \bar{z}, 0,0)+z \bar{z}(z \bar{z})^{|N|-1-i} \partial_{\bar{w}}^{|N|-1-i} \overline{\tilde{\psi}}_{\dot{+}}(z, \bar{z}, 0,0), \\
& \bar{\Psi}^{(i)}(z) \equiv \partial_{w}^{|N|-1-i} \tilde{\psi}_{+}(z, \bar{z}, 0,0)-z \bar{z}(z \bar{z})^{i} \partial_{\bar{w}}^{i} \bar{\psi}_{\dot{+}}(z, \bar{z}, 0,0),
\end{aligned}
$$

where $i=0,1, \cdots, N-1$. Again, $\bar{\Psi}(z)$ is not the complex conjugate of $\Psi(z)$. The OPE of the $4 \mathrm{~d}$ free fermions is given by

$$
\psi_{+}(z, \bar{z}, w, \bar{w}) \bar{\psi}_{\dot{+}}(0,0,0,0)=\frac{\bar{z}}{(z \bar{z}+w \bar{w})^{2}}+\cdots .
$$

Using this result, we obtain the $\Psi \bar{\Psi}$ OPE to be

$$
\Psi^{(i)}(z) \bar{\Psi}^{(j)}(0)=\frac{\delta^{i j}}{z}+\cdots
$$


where the $\bar{z}$-dependent terms are cancelled out. This is the OPE of $|N|$ chiral fermions in 2 d. So the chiral algebra is given by

$$
\mathcal{A}_{N}\left(\mathcal{T}_{H}\right)=\left\{\left\langle\Psi^{(i)}(z), \bar{\Psi}^{(i)}(z)\right\rangle \mid i=0, \cdots, N-1\right\} \quad \text { for } N>0,
$$

with the central charge $c^{2 d}=N$. Therefore the partition function of the chiral algebra is indeed given by the specialized index $\mathcal{I}_{N}^{H}=\theta\left(q^{\frac{1}{2}} u ; q\right)^{N} /(q)_{\infty}^{N}$.

Vector multiplets. When $N<0$, we define the chiral fields as

$$
\begin{aligned}
\partial c^{(0)}(z) & \equiv \partial_{w}^{|N|-1} \lambda_{1+}(z, \bar{z}, 0,0)+\bar{z} \lambda_{2+}(z, \bar{z}, 0,0), \\
b^{(0)}(z) & \equiv \lambda_{1 \dot{-}}(z, \bar{z}, 0,0)+\bar{z}(z \bar{z})^{|N|-1} \partial_{\bar{w}}^{|N|-1} \bar{\lambda}_{2 \dot{-}}(z, \bar{z}, 0,0),
\end{aligned}
$$

and

$$
\begin{aligned}
\partial c^{(i)}(z) & \equiv \partial_{w}^{i} \lambda_{1+}(z, \bar{z}, 0,0)+\bar{z}(z \bar{z})^{|N|-1-i} \partial_{\bar{w}}^{|N|-1-i} \lambda_{2+}(z, \bar{z}, 0,0), \\
b^{(i)}(z) & \equiv \partial_{w}^{|N|-1-i} \lambda_{1 \dot{+}}(z, \bar{z}, 0,0)+\bar{z}(z \bar{z})^{i} \partial_{\bar{w}}^{i} \bar{\lambda}_{2 \dot{+}}(z, \bar{z}, 0,0),
\end{aligned}
$$

with $1 \leq i \leq|N|-1$. Their OPE is the same as $|N|$ copies of the $b c$-system,

$$
b^{(i)}(z) c^{(j)}(0)=-c^{(i)}(z) b^{(j)}(0)=\frac{\delta^{i j}}{z}+\cdots
$$

So the chiral algebra for the vector multiplet can be written as

$$
\mathcal{A}_{N}\left(\mathcal{T}_{V}\right)=\left\{\left\langle b^{(i)}(z), c^{(i)}(z)\right\rangle|i=0, \cdots,| N \mid-1\right\} \quad \text { for } N<0
$$

It has the central charge $c^{2 d}=2 N=-2|N|$. At $N=-1$ only $b^{0}(z)$ and $c^{0}(z)$ remain and there is no derivative with respect to $w$ in the definition. In this case we again obtain the same $b c$-system obtained by Beem et al. [8] for vector multiplet.

When $N>0$ the we define the chiral fields as

$$
\begin{aligned}
\partial_{z} \Phi^{(i)}(z, 0) & \equiv \partial_{z} \partial_{w}^{i} \bar{\phi}(z, \bar{z}, 0,0)+\bar{z}^{2}(z \bar{z})^{|N|-1-i} \partial_{\bar{w}}^{|N|-1-i} F_{++}(z, \bar{z}, 0,0), \\
\partial_{z} \bar{\Phi}^{(i)}(z, 0) & \equiv \partial_{w}^{|N|-1-i} F_{--}(z, \bar{z}, 0,0)+\bar{z}^{2}(z \bar{z})^{i} \partial_{z} \partial_{\bar{w}}^{i} \phi(z, \bar{z}, 0,0),
\end{aligned}
$$

with $0 \leq i \leq N-1$. Their OPE can be computed as,

$$
\partial_{z} \Phi^{(i)}(z) \partial_{z} \bar{\Phi}^{(j)}(0)=\frac{\delta^{i j}}{z^{2}}+\cdots
$$

which is independent of $\bar{z}$. This is the same as the OPE of $N 2 d$ complex scalars. Therefore the chiral algebra is given by the $N$ complex scalars (in the adjoint of the gauge group)

$$
\mathcal{A}_{N}\left(\mathcal{T}_{V}\right)=\left\{\left\langle\Phi^{(i)}(z), \bar{\Phi}^{(i)}(z)\right\rangle \mid i=0, \cdots, N-1\right\} \quad \text { for } N>0
$$

with the central charge $c^{2 d}=2 N$.

We also see the origin of the zeroes and poles in the specialized index of vector multiplets. They are the zero modes of $b c$-system $(N<0)$ or complex scalars $(N>0)$. 


\subsubsection{Interacting theory}

Let us consider a Lagrangian theory $\mathcal{T}$ with gauge group $\mathcal{G}$ and hypermultiplets in $\mathcal{R} \times \mathcal{F}$ representation of gauge and flavor symmetry. We can construct the $2 \mathrm{~d}$ chiral algebra $\mathcal{A}_{N}$ with the partition function given by the specialized index $\mathcal{I}_{N}$ for any integer $N$. The construction is rather simple. We simply prepare the tensor product of the chiral algebras associated to each free matter multiplets and impose the Gauss law constraint. This will certainly reproduce the index $\mathcal{I}_{N}$.

For $N<0$ we get $|N| b c$-systems $b^{a,(i)}(z)$ and $c^{a,(i)}(z)$ with $i=0,1,2, \cdots,|N|-1$ in the adjoint representation of gauge group $\mathcal{G}$, and $|N|$ chiral symplectic bosons $Q^{(j)}(z)$ and $\bar{Q}^{(j)}(z)$ in representation $\mathcal{R} \times \mathcal{F}$. The chiral algebra is built upon the gauge invariant combination of $b^{a,(i)}(z), c^{a,(i)}(z), Q^{(j)}(z)$ and $\bar{Q}^{(j)}(z)$

$$
\mathcal{A}_{N}(\mathcal{T})=\left\{\left\langle b^{a,(i)}(z), c^{a,(i)}(z), Q^{(j)}(z), \bar{Q}^{(j)}(z)\right\rangle|i, j=0, \cdots,| N \mid-1\right\} / \mathcal{G}
$$

with $a$ being the index of adjoint representation and $i, j$ the label of different copies of the chiral fields.

For $N>0$ we get $N$ chiral scalars $\partial_{z} \Phi^{a,(i)}(z)$ and $\partial_{z} \bar{\Phi}^{a,(i)}(z)$ with $i=0,1, \cdots, N-1$ in the adjoint representation of gauge group $\mathcal{G}$, and $N$ chiral fermions $\Psi^{(i)}(z)$ and $\bar{\Psi}^{(i)}(z)$ in representation $\mathcal{R} \times \mathcal{F}$. The final $2 \mathrm{~d}$ algebra is built upon the gauge invariant combination of $\partial_{z} \Phi^{a,(i)}(z), \partial_{z} \bar{\Phi}^{a,(i)}(z), \Psi^{(i)}(z)$ and $\bar{\Psi}^{(i)}(z)$

$$
\mathcal{A}_{N}(\mathcal{T})=\left\{\left\langle\partial_{z} \Phi^{a,(i)}(z), \partial_{z} \bar{\Phi}^{a,(i)}(z), \Psi^{(j)}(z), \bar{\Psi}^{(j)}(z)\right\rangle \mid i, j=0, \cdots, N-1\right\} / \mathcal{G} .
$$

We constructed superconformal gauge theory out of the free matter content, and the index is obtained by imposing the Gauss law constraint. The chiral algebra at zero gauge coupling is constructed in this way and it certainly exists.

When the gauge coupling $g$ is non-zero, the chiral algebra has to be modified in a suitable way if it exists. In particular some elements of the algebra at $g=0$ will pair up and disappear at finite $g$, and it is natural to expect that generically there is a 1-1 correspondence between the elements of the algebra and the character for the algebra at $g>0$. Note that the partition function of the chiral algebra is robust because it is a limit of the superconformal index (with line operator insertion), which remains the same as we turn on the gauge coupling. Therefore we conjecture that there is still a chiral algebra even at finite coupling. For $N=-1$ the cohomological arguments of [8] imply that there is an algebra underlying the character at least for that case. We will find evidence by studying the Argyres-Douglas theories that the characters obtained by the monodromy operator are often characters of well known non-trivial 2d chiral algebras. This gives further evidence for our conjecture.

By construction, the central charge of the chiral algebra corresponding to the gauge theory is given (by considering the central charge of the integrand contributing to the index) by the formula

$$
c^{2 d}=N\left(n_{h}+2 n_{v}\right) .
$$

We see that it can be easily computed by enumerating number of vector multiplets $n_{v}$ and hypermultiplets $n_{h}$. For any $\mathcal{N}=2 d=4$ SCFT, one can define more invariant notions 
of the number of hypermultiplets and vector multiplets by considering conformal anomaly coefficients $a$ and $c$. It is given by

$$
n_{v}=4\left(2 a^{4 d}-c^{4 d}\right), \quad n_{h}=4\left(5 c^{4 d}-4 a^{4 d}\right) .
$$

From this relation, we get the $2 d$ central charge to be

$$
c^{2 d}=12 N c^{4 d}
$$

When $N=-1$, this is the same central charge given in [8]. We conjecture this relation holds even for the non-Lagrangian theories and for all $N$. We provide evidence for this in section 7 by computing $\operatorname{Tr} \mathcal{M}(q)^{N}$ for Argyres-Douglas theories of $\left(A_{1}, A_{n}\right)$ type for some values of $N$.

It is also easy to see that the effective growth of the states should be dictated for positive $N$ by $c^{2 d}$ but for $N<0$ just by considering the growth of the integrand, it is

$$
c_{\mathrm{eff}}^{2 d}=-2 N\left(n_{h}-n_{v}\right)=-48 N\left(c^{4 d}-a^{4 d}\right) \quad \text { for } N<0 .
$$

Assuming we have a chiral algebra, this implies that the minimum value of $h_{\min }$ for each $N<0$ is given by

$$
h_{\text {min }}=\frac{1}{24}\left(c^{2 d}-c_{\text {eff }}^{2 d}\right)=\frac{N}{2}\left(5 c^{4 d}-4 a^{4 d}\right)<0
$$

Note in particular for the Schur case of $N=-1$ we are predicting that there should be a representation of the chiral algebra of the Schur operators whose dimension $h=$ $\frac{1}{2}\left(4 a^{4 d}-5 c^{4 d}\right)$. For example for $\mathrm{SU}(2)$ with $N_{f}=4$ we expect $h_{\min }=-1$. The fact that $h_{\text {min }}$ should be negative for $N<0$ implies that $\left(5 c^{4 d}-4 a^{4 d}\right)>0$ which is consistent with the unitarity bound [22]. Moreover if we have a rational $2 \mathrm{~d}$ chiral algebra, it is expected that this combination is rational.

\subsubsection{Modular properties for conformal case}

A further evidence that we have a $2 \mathrm{~d}$ chiral algebra comes from the fact that the character is a nice modular object. Let us discuss modular properties of the specialized index $\mathcal{I}_{N}$. Before inserting any line operators, the specialized index can be written in terms of $\theta$ functions. We will see in section 5 that it is exactly the same as the integrand for the $S^{2} \times T^{2}$ partition function with $N$-twist (in the zero flux sector).

The specialized index for the hypermultiplet is given by

$$
\mathcal{I}_{N}^{H}=\prod_{w \in \mathcal{R}} \theta(\zeta \cdot w ; \tau)^{N}
$$

with $z_{i}=e^{2 \pi i \zeta_{i}}, \zeta \cdot w=\sum_{i=1}^{r} \zeta_{i} w^{i}$ and $q=e^{2 \pi i \tau}$. And for the vectors we have

$$
\mathcal{I}_{N}^{V}=\eta(q)^{-2 r N} \prod_{\alpha \in \Delta^{ \pm}(\mathcal{G})} \theta(\zeta \cdot \alpha ; \tau)^{-2 N}
$$

where $\Delta^{ \pm}(\mathcal{G})$ are the set of non-zero roots of the gauge group $\mathcal{G}$. 
The specialized index of a Lagrangian theory with zeroes or divergences removed is then essentially,

$$
\mathcal{I}_{n}=\eta^{-2 r N} \int d \zeta_{1} \cdots d \zeta_{r}\left(\prod_{w \in \mathcal{R}} \frac{\theta^{2}(\zeta \cdot w)}{\prod_{\alpha \in \Delta^{ \pm}(\mathcal{G})} \theta^{2}(\zeta \cdot \alpha)}\right)^{N},
$$

which is a Jacobi form. Note that we are being a bit sloppy in the above formulation because the boson versus fermions have different shifts in the argument of theta function. Under modular transformation $\zeta_{i}$ 's transform as $\zeta_{i} / \tau$ and the modular weight of $\eta^{2 r}$ cancels the weight of $\prod d \zeta_{i}$, leaving us with the modular weight of $\eta^{-2 r(N+1)}$ (which picks up a weight because of operator insertions when $N \neq-1$ ). Moreover in the Jacobi form the modular weight of the numerators cancels that of denominator if and only if

$$
\sum_{w \in \mathcal{R}}(\zeta \cdot w)^{2}-\sum_{\alpha \in \Delta^{ \pm}(\mathcal{G})}(\zeta \cdot \alpha)^{2}=0
$$

which is also the condition of vanishing $\beta$-function. Therefore the integrand of the specialized index is modular if and only if the Lagrangian theory is superconformal. As we shall discuss in section 5 , this is exactly the same as the condition that the $N / 2$-twisted compactification of the theory on $S^{2}$ to be free of gauge anomaly. Note that because we did not take into account the shifted arguments this is modular only on a subgroup of $\operatorname{SL}(2, \mathbb{Z})$. Moreover, even though the integrand is modular, the integral may not be modular because we have to change the integration contour by $\zeta_{i} \rightarrow \zeta_{i} / \tau$. The Schur index is given by the vacuum character of the chiral algebra for $N=-1$ [8]. It is not modular invariant, and will transform to different characters. Notable fact is that the modular transform for a Lagrangian theory is implemented by a change of contour. The partition function of the chiral algebra $\mathcal{A}_{N}$ for $N \neq-1$ is not written in terms of the $\theta$-functions, since we insert appropriate line operators to the integrand to remove zero modes. Therefore we do not expect it to be strictly modular. This is consistent with its transforming into combination of characters of modules of the same algebra as is expected for chiral algebras.

\subsection{Lagrangian examples}

\subsubsection{Abelian theories with matter}

Let us start with the simplest example of an interacting theory, even though it is only an effective theory. The index for the U(1) theory with a hypermultiplet can be evaluated easily by taking the index of a hypermultiplet and then integrating over $\mathrm{U}(1)$ gauge group. It is given by

$$
\mathcal{I}(p, q, t)=\mathcal{I}^{V} \oint \frac{d z}{2 \pi i z} \prod_{i, j \geq 0}\left(\frac{1-t^{-\frac{1}{2}} p^{i+1} q^{j+1} z}{1-t^{\frac{1}{2}} p^{i} q^{j} z}\right)\left(\frac{1-t^{-\frac{1}{2}} p^{i+1} q^{j+1} z^{-1}}{1-t^{\frac{1}{2}} p^{i} q^{j} z^{-1}}\right)
$$

where $\mathcal{I}^{V}$ is the free $\mathrm{U}(1)$ vector multiplet index. The integrand in the limit $t=q p^{N+1}$ can be simply written as in (4.10). Also, if we take $p \rightarrow 1$, we simply get

$$
\mathcal{I}_{N}(q)=\mathcal{I}_{N}^{V} \oint \frac{d z}{2 \pi i z}\left(q^{\frac{1}{2}} z^{ \pm} ; q\right)^{N}
$$


The chiral algebra is simply given by that of the free fermions $(N>0)$ or bosons $(N<0)$ under the Gauss law constraint (along with the decoupled piece coming from the free vector multiplets)

$$
\mathcal{A}_{N}\left(\mathcal{T}_{Q E D}\right)=\left\{\begin{array}{l}
\mathcal{A}_{N}\left(\mathcal{T}_{V}\right) \otimes\left\{\left\langle\Psi^{i}(z), \bar{\Psi}^{i}(z) \mid i=0,1, \cdots, N\right\rangle\right\} / \mathrm{U}(1) \quad N>0, \\
\mathcal{A}_{N}\left(\mathcal{T}_{V}\right) \otimes\left\{\left\langle Q^{i}(z), \bar{Q}^{i}(z)\right\rangle \mid i=0,1, \cdots, N\right\} / \mathrm{U}(1) \quad N<0 .
\end{array}\right.
$$

Index for $\boldsymbol{N}>\mathbf{0}$. Let us compute the specialized indices $\mathcal{I}_{N}$ (equivalently the character of the chiral algebra $\mathcal{A}_{N}\left(\mathcal{T}_{Q E D}\right)$ ) for $N>0$. The integral (4.59) can be evaluated (with suitable insertion of operators already discussed to absorb the bosonic zero modes) to give

$$
\mathcal{I}_{N}(q)=\mathcal{I}_{N}^{V} \times \frac{1}{(q)_{\infty}^{N}} \oint \frac{d z}{2 \pi i z} \sum_{\vec{k} \in \mathbb{Z}^{N}} q^{\frac{1}{2} \sum_{i} k_{i}^{2}}(-z)^{\sum_{i} k_{i}}=\mathcal{I}_{N}^{V} \times \frac{1}{(q)_{\infty}^{N}} \sum_{\vec{k} \in \mathbb{Z}^{N-1}} q^{Q(\vec{k})}
$$

where

$$
Q(\vec{k})=\sum_{i} k_{i}^{2}+\sum_{i<j} k_{i} k_{j}
$$

By general theory of integral (Tits) quadratic forms [23], $Q\left(k_{i}\right)$ is $\mathbb{Z}$-equivalent to $\ell_{i} C_{i j} \ell_{j} / 2$ where $C_{i j}$ is the $A_{N-1}$ Cartan matrix, i.e. there is a transformation $\ell_{i}=S_{i j} k_{j}$, with $S \in \mathrm{SL}(N-1, \mathbb{Z})$ such that $Q\left(S_{i j}^{-1} \ell_{j}\right) \equiv \ell_{i} C_{i j} \ell_{j} / 2$; explicitly, $\ell_{i}=k_{i}-k_{i-1}$ with the convention $k_{0}=0$. Hence

$$
\sum_{\vec{k} \in \mathbb{Z}^{N-1}} q^{Q(\vec{k})}=\sum_{\ell_{i} \in \mathbb{Z}^{N-1}} q^{\ell_{i} C_{i j} \ell_{j} / 2} \equiv \Theta_{\mathrm{SU}(N)}(q)
$$

Therefore, we get

$$
\mathcal{I}_{N}(q)=\mathcal{I}_{N}^{V} \times \frac{\Theta_{\mathrm{SU}(N)}(q)}{(q ; q)_{\infty}^{N}}=\frac{\Theta_{\mathrm{SU}(N)}(q)}{(q ; q)_{\infty}^{3 N}} .
$$

The central charge is $c^{2 d}=2 N+N=3 N$, where the first $2 N$ is coming from the decoupled vector multiplet, which becomes $N$ free complex bosons.

Index for $\boldsymbol{N}<\mathbf{0}$. When $N=-1$, we get the Schur index. We obtain

$$
\mathcal{I}_{-1}(q)=\mathcal{I}_{-1}^{V} \times \frac{\Psi(q)}{(q ; q)_{\infty}^{2}}=\Psi(q)
$$

where $\Psi(q)=\frac{1}{2} \sum_{n \in \mathbb{Z}} q^{\frac{n(n+1)}{2}}$. Note that even though we needed no insertion for $N=-1$ this is not a modular weight zero object. This is consistent with our analysis that showed that only for superconformal theories we expect to get a modular weight zero object (at $N=-1)$. Nevertheless the theta function is a modular object with weight. The central charge for the chiral algebra is $c_{\text {tot }}^{2 d}=-2+2=0$. The first term -2 is coming from the free vector and the latter 2 is the one coming from the hypermultiplets under the Gauss law constraint. For the case of $N=-2$, we find

$$
\mathcal{I}_{-2}(q)=\mathcal{I}_{-2}^{V} \times \frac{1}{(q ; q)_{\infty}^{4}} \sum_{n \geq 0}(2 n+1) q^{n^{2}+n+1}
$$

We get the central charge as $c_{\text {total }}^{2 d}=-4+4=0$ if we also include the vector multiplet. 
For general $N<0$, with the help of identities in appendix D.1, we get

$$
\begin{aligned}
\mathcal{I}_{N}(q) & =\mathcal{I}_{-|N|}^{V} \times \frac{1}{(q ; q)_{\infty}^{2|N|}} \sum_{m_{i} \in \mathbb{Z}^{|N|-1}} q^{\left(\left|\sum_{i} m_{i}\right|+\sum_{i}\left|m_{i}\right|\right) / 2} \psi\left(-q^{\left|\sum_{i} m_{i}\right|}, q\right) \prod_{i=1}^{|N|-1} \psi\left(-q^{\left|m_{i}\right|}, q\right) \\
& =\mathcal{I}_{-|N|}^{V} \times \frac{\Psi_{|N|}(q)}{(q ; q)_{\infty}^{2|N|}} \equiv \Psi_{|N|}(q)
\end{aligned}
$$

where

$$
\psi(x, q)=\sum_{m \geq 0} q^{m(m+1) / 2} x^{m}
$$

is Ramanujan's partial theta-function. The function $\Psi_{N}(q)$ is the sum of $2^{|N|-1}$ multiple partial theta-functions, or more compactly

$$
\Psi_{N}(q)=\sum_{\substack{n_{i} \in \mathbb{N}^{N} \\ m_{j} \in \mathbb{Z}^{N-1}}}(-1)^{\sum_{i=1}^{N} n_{i}} q^{Q\left(m_{i}, n_{j}\right) / 2+\sum_{i}\left(\left|m_{i}\right|+n_{i}\right) / 2},
$$

where $Q\left(m_{i}, n_{j}\right)$ is the quadrant-wise quadratic form

$$
Q\left(m_{i}, n_{j}\right)=\sum_{j=1}^{N} n_{j}^{2}+2 \sum_{i=1}^{N-1} n_{i}\left|m_{i}\right|+2 n_{N}\left|\sum_{i=1}^{N-1} m_{i}\right| .
$$

The sum is a (multiple) partial theta function. We get the central charge $c_{\text {total }}^{2 d}=-2 N+$ $2 N=0$, if we include the vector multiplet contribution.

\subsubsection{U(1) theory with $N_{f}$ hypermultiplets}

Let us consider a slightly more general case.

Index for $\boldsymbol{N} \geq \mathbf{0}$. Suppose we have several hypers of integral charges $e_{a}$ and fugacities $y_{a}\left(a=1,2, \ldots, N_{f}\right)$ (one redundant). For $N \geq 0$,

$$
\mathcal{I}_{N}\left(q ; y_{a}\right)=\frac{1}{(q)_{\infty}^{N\left(N_{f}+2\right)}} \int \frac{d z}{2 \pi i z} \prod_{a=1}^{N_{f}} \Theta\left(-y_{a} z^{e_{a}} ; q\right)^{N}=\frac{\Theta_{N}\left(e_{a}, y_{a} ; q\right)}{(q)_{\infty}^{N\left(N_{f}+2\right)}}
$$

where $\Theta_{N}\left(e_{a}, y_{a} ; q\right)$ is the theta-function of the rank $N N_{f}-1$ positive-definite sub-lattice

$$
\Lambda_{N}\left(\left\{e_{a}\right\}\right)=\left\{k_{a, i} \in \mathbb{Z}^{N_{f}} \times \mathbb{Z}^{N} \mid \sum_{i, a} e_{a} k_{i, a}=0\right\} \subset \mathbb{Z}^{n N_{f}},
$$

endowed with the quadratic form $Q$ induced by the standard one in $\mathbb{Z}^{N N_{f}}, \sum_{i, a} k_{a, i}^{2}$, i.e.

$$
\Theta_{N}\left(e_{a}, y_{a} ; q\right)=\sum_{k_{i, a} \in \Lambda_{N}\left(\left\{e_{a}\right\}\right)} q^{\sum_{i, a} k_{a, i}^{2} / 2} \prod_{a}\left(-y_{a}\right)^{\sum_{i} k_{i, a}} .
$$

In particular, if all $e_{a}$ are equal, we get the $N_{f}-1$ variable specialization of the $\mathrm{SU}\left(N N_{f}\right)$ theta-function induced by (and covariant under) the subgroup inclusion

$$
\mathrm{SU}\left(N_{f}\right) \times \mathrm{SU}(N) \subset \mathrm{SU}\left(N_{f} N\right)
$$


Of course, this is just the statement that supersymmetric quantum electrodynamics (SQED) with $N_{f}$ quarks of the same charge has a $\mathrm{SU}\left(N_{f}\right)$ symmetry: indeed, via the replica trick, the integral (4.71) is identified with the one entering in the $N=1$ index for SQED with $N N_{f}$ quarks which has a $\mathrm{SU}\left(N N_{f}\right)$ flavor symmetry and therefore produces the $\mathrm{SU}\left(N N_{f}\right)$ theta-function specialized to the locus in fugacity space which invariant under the $\mathrm{SU}(N)$ replica symmetry. In the basic case that all $e_{a}$ are equal and $N=1$ we get the full $\mathrm{SU}\left(N_{f}\right)$ theta-function depending on all its $N_{f}-1$ fugacities.

Even more generally, we may couple $k$ Abelian vectors to $N_{f}$ hypers the $a$-th hyper having (integral) charge $e_{a, \alpha}$ under the $\alpha$-th photon $(\alpha=1, \ldots, k)$. For $n \geq 0$ we get

$$
\mathcal{I}_{N}\left(q ; y_{a}\right)=\frac{\Theta_{N}\left(e_{a, \alpha}, y_{a} ; q\right)}{(q)_{\infty}^{N\left(N_{f}+2 k\right)}}
$$

where in the numerator we have the obvious flavor group covariant specialization of the theta function for the rank $N N_{f}-k$ lattice

$$
\Lambda_{N}\left(\left\{e_{a, \alpha}\right\}\right)=\left\{k_{a, i} \in \mathbb{Z}^{N_{f}} \times \mathbb{Z}^{N} \mid \sum_{i, a} e_{a, \alpha} k_{i, a}=0 \text { for } \alpha=1, \ldots, k\right\} \subset \mathbb{Z}^{N N_{f}} .
$$

Index for $\boldsymbol{N}<\mathbf{0}$. When $N<0$, we have

$$
\mathcal{I}_{-N}\left(q ; y_{a}\right)=\frac{1}{(q)_{\infty}^{2|N|\left(N_{f}-k\right)}} \int \prod_{\alpha=1}^{k} \frac{d z_{\alpha}}{2 \pi i z_{\alpha}} \prod_{a=1}^{N_{f}} \Xi\left(y_{a} z_{\alpha}^{e_{a, \alpha}} ; q\right)^{|N|}=\frac{\Psi_{|N|}\left(e_{a, \alpha}, y_{a} ; q\right)}{(q)_{\infty}^{2|N|\left(N_{f}-k\right)}}
$$

where $\Xi(z ; q)$ is the function

$$
\Xi(z ; q)=\frac{(q)_{\infty}^{2}}{\left(q^{1 / 2} z ; q\right)_{\infty}\left(q^{1 / 2} z^{-1} ; q\right)_{\infty}}
$$

which may be written as the sum of partial theta-functions in the form (see appendix D.1) ${ }^{6}$

$$
\Xi(z ; q)=\sum_{n \in \mathbb{Z}} q^{|n| / 2} \psi\left(-q^{|n|}, q\right) z^{n} \equiv \sum_{\substack{n \in \mathbb{Z} \\ m \geq 0}}(-1)^{m} q^{m(m+1) / 2+|n|(m+1 / 2)} z^{n} .
$$

The function $\Psi_{N}\left(e_{a, \alpha}, y_{a} ; q\right)$ is the sum of $2^{N N_{f}-k}$ partial theta functions which is invariant under the $\mathrm{SU}(N)$ replica symmetry (which acts on the replica index $i=1, \ldots, N$ )

$$
\Psi_{N}\left(e_{a, \alpha}, y_{a} ; q\right)=\sum_{n_{a, i} \in \Lambda_{N}\left(\left\{e_{a, \alpha}\right\}\right)_{m_{a, i} \in \mathbb{N}^{N N_{f}}}}(-1)^{\sum_{a, i} m_{a, i}} q^{\frac{Q\left(m_{a, i}, n_{a, i}\right)}{2}+\sum_{a, i} \frac{m_{a, i}+\left|n_{a, i}\right|}{2}} \prod_{a} y_{a}^{\sum_{i} n_{a, i}}
$$

where $Q\left(m_{a, i}, n_{a, i}\right)$ is the quadrant-wise quadratic form

$$
Q\left(m_{a, i}, n_{a, i}\right)=\sum_{a, i} m_{a, i}^{2}+2 \sum_{a, i}\left|m_{a, i}\right| n_{a, i} .
$$

\footnotetext{
${ }^{6}$ The right-hand side of this expression has to be understood with care, because the summand is not absolutely convergent and the ordering is important. Here the sum over $m \geq 0$ has to be taken first. We thank O. Warnaar for the comment.
} 


\subsubsection{Pure SU(2)}

Let us consider the case for the pure SU(2) Yang-Mills theory. This theory is not superconformal away from $g_{Y M}=0$, and we restrict our attention to this point.

Specialized index at $N=-\mathbf{1}$. At $N=-1$ the specialized index is the same as Schur index and for the $\mathrm{SU}(2) \mathrm{YM}$ it is given by

$$
\begin{aligned}
\mathcal{I}_{-1}^{S Y M}(q) & =\oint \frac{d z}{2 \pi i z} \frac{\left(1-z^{ \pm 2}\right)}{2}\left(q z^{ \pm 2,0} ; q\right)_{\infty}^{2} \\
& =\sum_{n=0}^{\infty} q^{n(n+1)}=1+q^{2}+q^{6}+q^{12}+\cdots .
\end{aligned}
$$

It can be easily proven by using the Jacobi triple product identity $(q ; q)_{\infty}(y ; q)_{\infty}(q / y ; q)_{\infty}=$ $\sum_{k \in \mathbb{Z}} q^{k(k+1) / 2} y^{-k}$ as

$$
\begin{aligned}
\mathcal{I}_{-1}^{S Y M}(q) & =\frac{1}{2} \oint \frac{d z}{2 \pi i z}(q ; q)_{\infty}\left(z^{2} ; q\right)_{\infty}\left(q / z^{2} ; q\right)_{\infty}(q ; q)_{\infty}\left(z^{-2} ; q\right)_{\infty}\left(q z^{2} ; q\right)_{\infty} \\
& =\frac{1}{2} \sum_{m, n \in \mathbb{Z}} q^{\frac{1}{2} m(m+1)+\frac{1}{2} n(n+1)} \oint \frac{d z}{2 \pi i z} z^{2(m-n)}=\sum_{n \geq 0} q^{n(n+1)} .
\end{aligned}
$$

This is the character of the chiral algebra

$$
\left.\mathcal{A}_{-1}\left(\mathcal{T}_{\mathrm{SU}(2)}\right)\right|_{g=0}=\left\{\left\langle\partial b^{a}(z), \partial c^{a}(z)\right\rangle\right\} / \mathrm{SU}(2),
$$

where $a$ denotes the gauge index. This is the singlet sector of the $b c$-system in the adjoint representation of $\mathrm{SU}(2)$.

Specialized index at $\mathbf{N}=\mathbf{- 2}$. The integral formula with line operators inserted for $N=-2$ specialized index is given by

$$
\mathcal{I}_{-2}^{S Y M}(q)=\oint \frac{d z}{2 \pi i z} \frac{\left(1-z^{ \pm 2}\right)}{2}\left(q z^{ \pm 2,0} ; q\right)_{\infty}^{4} .
$$

Expanding in powers of $q$ we have,

$$
\mathcal{I}_{-2}^{S Y M}(q)=1+6 q^{2}-4 q^{3}+3 q^{4}+12 q^{5}-2 q^{6}-12 q^{7}+18 q^{8}+8 q^{9}+12 q^{10}+\cdots .
$$

The chiral algebra is built upon the gauge invariant combinations of two $b c$-systems in the adjoint representation of $\mathrm{SU}(2)$ with the zero modes $b^{a}(0)$ and $c^{a}(0)$ removed,

$$
\left.\mathcal{A}_{-2}\left(\mathcal{T}_{\mathrm{SU}(2)}\right)\right|_{g=0}=\left\{\left\langle\partial b^{a, 0}(z), \partial c^{a, 0}(z), \partial b^{a, 1}(z), \partial c^{a, 1}(z)\right\rangle\right\} / \mathrm{SU}(2) .
$$

The partition function counts the number of operators with sign. Let us illustrate this by explicitly counting operators for low orders in $q$. In the weak coupling limit, the $6 q^{2}$ term counts six operators

$$
\operatorname{Tr}_{a} \partial b^{i} \partial c^{j}, \quad \operatorname{Tr}_{a} \partial b^{1} \partial b^{2}, \quad \operatorname{Tr}_{a} \partial c^{1} \partial c^{2},
$$

with $\operatorname{Tr}_{a}$ the trace over adjoint representation of $\mathrm{SU}(2)$. The $-4 q^{3}$ term counts 16 bosonic operators

$$
\operatorname{Tr}_{a} \partial^{2} b^{i} \partial b^{j}, \quad \operatorname{Tr}_{a} \partial^{2} b^{i} \partial c^{j}, \quad \operatorname{Tr}_{a} \partial^{2} c^{i} \partial b^{j}, \quad \operatorname{Tr}_{a} \partial^{2} c^{i} \partial c^{j},
$$


and 20 fermionic operators

$$
\epsilon_{a b c} \partial b^{a, i} \partial b^{b, j} \partial b^{c, k}, \quad \epsilon_{a b c} \partial b^{a, i} \partial b^{b, j} \partial c^{c, k}, \quad \epsilon_{a b c} \partial b^{a, i} \partial c^{b, j} \partial c^{c, k}, \quad \epsilon_{a b c} \partial c^{a, i} \partial c^{b, j} \partial c^{c, k} .
$$

There is no a priori reason that in a non-conformal theory, such as pure $\mathrm{SU}(2)$ the theory is conformal away from $g=0$. However, if the algebra continued to exist beyond $g=0$ we would have expected that the 16 bosonic states would pair up with 16 of the fermionic states leaving us with 4 fermionic operators.

Specialized index at $\boldsymbol{N}=\mathbf{1}$. The integral formula with the line operator inserted for $N=1$ specialized index is given by

$$
\mathcal{I}_{1}^{S Y M}(q)=\oint \frac{d z}{2 \pi i z} \frac{\left(1-z^{ \pm 2}\right)}{2} \frac{1}{\left(q z^{ \pm 2,0} ; q\right)_{\infty}^{2}} .
$$

Expanding in powers of $q$ we have,

$$
\mathcal{I}_{1}^{S Y M}(q)=1+3 q^{2}+4 q^{3}+15 q^{4}+24 q^{5}+\cdots .
$$

Note that all the coefficients are positive integers. The chiral algebra is built upon the gauge invariant combinations of a complex chiral bosons $\Phi^{a}(z)$ and $\bar{\Phi}^{a}(z)$ in adjoint representation of $\mathrm{SU}(2)$ with the zero modes $\Phi^{a}(0)$ removed,

$$
\left.\mathcal{A}_{1}\left(\mathcal{T}_{\mathrm{SU}(2)}\right)\right|_{g=0}=\left\{\left\langle\partial \Phi^{a}(z), \partial \bar{\Phi}^{a}(z)\right\rangle\right\} / \mathrm{SU}(2)
$$

For example in the weakly coupled limit, $3 q^{2}$ term counts three operators $\operatorname{Tr}_{a} \partial \Phi \partial \Phi$, $\operatorname{Tr}_{a} \partial \Phi \partial \bar{\Phi}$ and $\operatorname{Tr}_{a} \partial \bar{\Phi} \partial \bar{\Phi}$. Since there is no fermionic operator, coefficients of $\mathcal{I}_{1}(q)$ are always greater than equal to zero.

\subsection{4 $\mathrm{SU}(2)$ with $N_{f}=4$}

Specialized index at $\mathbf{N}=\mathbf{- 1}$. This is the same as Schur index. In the unrefined limit where we turn off all the flavor fugacities, we get

$$
\mathcal{I}_{-1}(q)=\oint \frac{d z}{2 \pi i z} \frac{\left(1-z^{ \pm 2}\right)}{2} \frac{\left(q z^{ \pm 2,0} ; q\right)_{\infty}^{2}}{\left(\left(q^{\frac{1}{2}} z^{ \pm 1} ; q\right)_{\infty}^{2}\right)^{4}},
$$

with the expansion

$$
\mathcal{I}_{-1}=1+28 q+329 q^{2}+2632 q^{3}+16380 q^{4}+85764 q^{5}+\cdots .
$$

This is the character of $\mathrm{SO}(8)_{-2}$ according to [8]. The chiral algebra $\mathcal{A}_{-1}\left(\mathcal{T}_{\mathrm{SU}(2), N_{f}=4}\right)=$ $\mathrm{SO}(8)_{-2}$ has the central charge equal to that of the Sugawara central charge $c^{2 d}=-14$.

Specialized index at $\boldsymbol{N}=\mathbf{1}$. The specialized index at $N=1$ with line operator inserted (in the unrefined limit) is,

$$
\mathcal{I}_{1}(q)=\oint \frac{d z}{2 \pi i z} \frac{\left(1-z^{ \pm 2}\right)}{2} \frac{\left(\left(q^{\frac{1}{2}} z^{ \pm 1} ; q\right)^{2}\right)_{\infty}^{4}}{\left(q z^{ \pm 2,0} ; q\right)_{\infty}^{2}}
$$


and its expansion in $q$ is

$$
\mathcal{I}_{1}(q)=1+36 q+459 q^{2}+3700 q^{3}+23403 q^{4}+125232 q^{5}+\cdots .
$$

The chiral algebra is built upon the gauge invariant combinations of one complex boson $\Phi(z)$ in adjoint representation of $\mathrm{SU}(2)$ and four complex bifundamental fermion $\Psi(z)$,

$$
\mathcal{A}_{1}\left(\mathcal{T}_{\mathrm{SU}(2), N_{F}=4}\right)=\left\{\left\langle\partial \Phi^{a}(z), \partial \bar{\Phi}^{a}(z), \Psi(z), \bar{\Psi}(z)\right\rangle\right\} / \mathrm{SU}(2)
$$

where we remove the zero modes $\Phi^{a}(0)$ and $\bar{\Phi}^{a}(0)$. Here $\Psi^{i}(z)$ is in the $(2,4)$ representation of $\mathrm{SU}(2)_{\mathcal{G}} \times \mathrm{U}(4)_{F}$ and $\bar{\Psi}^{i}(z)$ is in the $(2, \overline{4})$ representation of $\mathrm{SU}(2)_{\mathcal{G}} \times \mathrm{U}(4)_{F}$.

The term $36 q$ in the index comes from the gauge invariant combination of $\Psi^{i}(0) \Psi^{j}(0)$, $\Psi^{i}(0) \bar{\Psi}^{j}(0)$ and $\bar{\Psi}^{i}(0) \bar{\Psi}^{j}(0)$, which form the adjoint representation of USp(8) having dimension 36. Moreover, it has correct decomposition under the flavor symmetry $\mathrm{SU}(4) \times \mathrm{U}(1) \subset$ $\mathrm{USp}(8)$. It shows that there is an $\operatorname{USp}(8)$ subalgebra. For $N>1$, we have $N$ copies $\Psi(z)$ and $\bar{\Psi}(z)$. We find that the coefficient of $q$ in the index $\mathcal{I}_{N}(q)$ is given by the dimension of the adjoint representation of $\operatorname{USp}(8 N)$, which is $4 N(8 N+1)$.

Specialized index at $N=\mathbf{- 2}$. Let us consider the case with $N=-2$. The specialized index at $N=-2$ is given by

$$
\mathcal{I}_{-2}(q)=\oint \frac{d z}{2 \pi i z} \frac{\left(1-z^{ \pm 2}\right)}{2} \frac{\left(q z^{ \pm 2,0} ; q\right)_{\infty}^{4}}{\left.\left(q^{\frac{1}{2}} z^{ \pm} ; q\right)_{\infty}^{4}\right)^{4}},
$$

where its expansion in $q$ is

$$
\mathcal{I}_{-2}(q)=1+120 q+5158 q^{2}+124644 q^{3}+2065459 q^{4}+26107916 q^{5}+\cdots .
$$

The chiral algebra is constructed from the two copies of the $b c$-system $\left(b^{a, i=0,1}, c^{a, i=0,1}\right)$ in the adjoint representation of $\mathrm{SU}(2)_{\mathcal{G}}$ and two copies of the symplectic bosons $Q^{i=0,1}$ and $\bar{Q}^{i=0,1}$ in the $(2,4)$ and $(2, \overline{4})$ representations of $\mathrm{SU}(2)_{\mathcal{F}} \times \mathrm{U}(4)_{F}$. Then we impose the Gauss-law constraint to build

$$
\mathcal{A}_{-2}\left(\mathcal{T}_{\mathrm{SU}(2), N_{F}=4}\right)=\left\{\left\langle\partial b^{a, i}(z), \partial c^{a, i}(z), Q^{i}(z), \bar{Q}^{i}(z) \mid i=0,1\right\rangle\right\} / \mathrm{SU}(2),
$$

where we removed the zero modes in the $b c$-system.

The term $120 q$ in the index comes from the gauge invariant combinations of $Q^{i}(0) \bar{Q}^{j}(0)$, $Q^{i}(0) \bar{Q}^{j}(0)$ and $\bar{Q}^{i}(0) \bar{Q}^{j}(0)$. They form the adjoint representation of $\mathrm{SO}(16)$ having dimension 120 . We see that for general $N<0$, the coefficient of the $q$ term is given by the dimension of the adjoint representation of $\mathrm{SO}(-8 N)$, which is $4|N|(8|N|-1)$.

\section{$5 \quad T^{2} \times S^{2}$ compactifications}

Up to now we have discussed a specialization of the index and how to compute it in Lagrangian theories. It turns out, somewhat surprisingly, that almost the same result arises from a suitable compactification of the same theory on $S^{2}$ and considering its partition function on $T^{2}$. We will find that this gives the same integrands as the ones appearing in 
the index computation, even though the contour prescriptions for the integrals are slightly different. The nice aspect of this correspondence is that in this context the $T^{2}$ is the relevant physical space upon taking the limit where $S^{2}$ goes to zero size, and not just part of the $S^{3} \times S^{1}$ geometry which is somewhat harder to visualize. Moreover in this set up we can see more clearly the meaning of fermionic zero modes for $N<-1$ and bosonic zero modes for $N>0$. In this context it turns out that one can add a chemical potential associated with the $\mathrm{U}(1)$ rotation of $S^{2}$ which gets rid of zero modes. We stress that this construction describes a full physical theory, and not just the supersymmetry protected states that contribute to the elliptic genus. In a sense we get a $(0,2)$ SCFT in the infrared (IR), whose elliptic genus we are computing.

\section{$5.12 \mathrm{~d} \mathcal{N}=(0,2)$ theory from twisted compactification on $S^{2}$}

Let us consider putting the $4 \mathrm{~d} \mathcal{N}=2$ superconformal theory on $\mathbb{R}^{2} \times S^{2}$. In order to preserve any amount of supersymmetry, we need to perform a partial topological twist [24]. The bosonic subgroup of the $4 \mathrm{~d} \mathcal{N}=2$ superconformal group includes $\mathrm{SO}(4) \times \mathrm{SU}(2)_{R} \times \mathrm{U}(1)_{r}$ where $\mathrm{SO}(4)$ is the (Euclidean) Lorentz group acting on the spacetime. Let us consider the subgroup $\mathrm{SO}(2)_{E} \times \mathrm{SO}(2)_{S^{2}} \subset \mathrm{SO}(4)$. The first factor acts on $\mathbb{R}^{2}$ and the second factor acts on $S^{2}$. We can twist the theory by considering a linear combination of $\mathrm{SU}(2)_{R}$ and $\mathrm{U}(1)_{r}$

$$
J_{34}^{(N)}=J_{34}+\left(1+\frac{1}{2} N\right) R-\frac{1}{2} N r=J_{34}+R+\frac{1}{2} N(R-r),
$$

where $J_{34}, R, r$ are the generators of $\mathrm{SO}(2)_{S^{2}}, \mathrm{SU}(2)_{R}$, and $\mathrm{U}(1)_{r}$ respectively. When $N=0$, the twisted theory preserves $\mathcal{N}=(2,2)$ SUSY in 2 d. When $N=-2$, it preserves $\mathcal{N}=(0,4)$ SUSY in $2 \mathrm{~d}[25]$ and it has been considered in [26]. See appendix A for details.

Upon twisting and dimensional reduction, we obtain an effective $2 \mathrm{~d} \mathcal{N}=(0,2)$ theory on $\mathbb{R}^{2}$. We can be very explicit for the free theory of hypermultiplet or vector multiplet. The $4 \mathrm{~d}$ vector multiplet becomes $\mathcal{N}=(0,2)$ vector multiplet and $N+1$ chiral multiplets when $N>0$ and becomes vector and $|N|-1$ Fermi multiplets for $N<0$. A 4 d hypermultiplet becomes $N$ Fermi multiplets for $N>0$ and $N$ chiral multiplets when $N<0$. The astute reader may have noticed that our twisting for odd values of $N$ is somewhat problematic because some fields end up having fractional spin and so we would need a further twist by some global symmetry to make sense of them. For odd $N$, this can only be done if we have an additional $\mathrm{U}(1)$ global symmetry which distinguish $Q$ and $\widetilde{Q}$ in a hypermultiplet. In this case, we need to further twist the theory by this $\mathrm{U}(1)$ [27]. This can be always done for a Lagrangian CFT by breaking some of the global symmetry in $4 \mathrm{~d}$.

Let us start from a $4 \mathrm{~d} \mathcal{N}=2 \mathrm{SCFT}$ realized as a gauge theory given by gauge group $\mathcal{G}$ with hypermultiplets in some representation $\mathcal{R}_{i}$ of $\mathcal{G} \times \mathcal{F}$ where $\mathcal{F}$ is the flavor symmetry group. ${ }^{7}$ For this class of theories, the gauge couplings are exactly marginal, so we can continuously deform the theory to the zero coupling limit. At this point, we perform partial topological twisting and shrink the size of $S^{2}$ to zero. Then we obtain the matter content in table 1.

\footnotetext{
${ }^{7}$ Such $\mathcal{N}=2$ SCFTs are classified in the paper [28].
} 


\begin{tabular}{|c|c|}
\hline $4 \mathrm{~d} \mathcal{N}=2$ multiplet & $2 \mathrm{~d} \mathcal{N}=(0,2)$ multiplets \\
\hline vector multiplet & 1 vector and $\begin{cases}N+1 & \text { chiral multiplets } \\
-(N+1)=|N|-1 \text { Fermi multiplets } & (N<0)\end{cases}$ \\
\hline hypermultiplet & $\left\{\begin{array}{lll}N & \text { Fermi multiplets } & (N>0) \\
|N| & \text { chiral multiplets } & (N<0)\end{array}\right.$ \\
\hline
\end{tabular}

Table 1. $4 \mathrm{~d} \mathcal{N}=2$ matter multiplets in terms of $2 \mathrm{~d} \mathcal{N}=(0,2)$ multiplets after twisting.

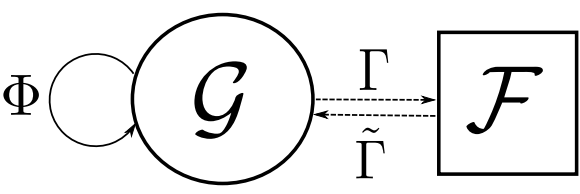

(a) $N>0$ : there are $N+1$ copies of $\Phi$ and $N / 2$ copies of $(\Gamma, \tilde{\Gamma})$.

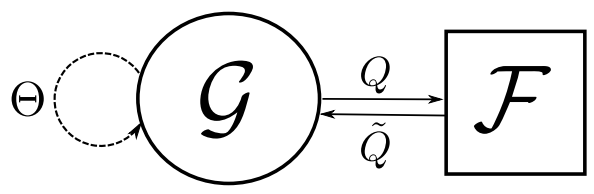

(b) $N<0$ : there are $|N|-1$ copies of $\Theta$ and $|N| / 2$ copies of $(Q, \tilde{Q})$.

Figure 1. Schematic matter content of the 2 d gauge theory obtained by twisted reduction on $S^{2}$. $\mathcal{G}$ denotes the gauge group and $\mathcal{F}$ denotes the flavor group.

When $N<0$, we get $\mathcal{N}=(0,2)$ gauge theory with the same gauge group $\mathcal{G}$ but $(|N|-1)$ copies of the Fermi multiplets $(\Theta)$ in the adjoint of $\mathcal{G}$ and $N / 2$ copies of chiral multiplets $(Q, \tilde{Q})$ in the $\mathcal{R}_{i}$ and also its conjugate $\overline{\mathcal{R}}_{i}$ representation of $\mathcal{G} \times \mathcal{F}$. In the parenthesis, we write $\mathcal{N}=(0,2)$ superfields for each multiplet. We have both $\mathcal{R}_{i}$ and $\overline{\mathcal{R}}_{i}$ because the original $4 \mathrm{~d}$ theory had chiral multiplets in the same representations. When $N$ is odd, we only have $\mathcal{R}_{i}$ representation or its conjugate by further twisting by the baryonic charge. In addition, we have a $J$-type superpotential interaction term inherited from $4 \mathrm{~d} \mathcal{N}=2$ given by

$$
J_{\Theta}=\tilde{Q} Q \quad \rightarrow \quad W=\operatorname{Tr}(\tilde{Q} \Theta Q)
$$

where we suppressed indices of the $|N| / 2$ copies of $Q, \tilde{Q}$ and $|N|-1$ copies of $\Theta$. One can impose $\mathrm{SU}(|N| / 2)$ symmetry rotating among the $\Phi$ along with $Q$, by tuning the coupling, but we will not enforce it in this discussion. Note that when $N=-2$, this is exactly the superpotential and matter content to preserve $\mathcal{N}=(0,4)$ supersymmetry discussed in [26].

When $N>0$, we basically reverse the Fermi and chiral multiplets. We get $N+1$ copies of the chiral multiplets $(\Phi)$ in the adjoint representation of $\mathcal{G}$, and $N / 2$ copies of chiral multiplets $(\Gamma, \tilde{\Gamma})$ in the $\mathcal{R}_{i}$ and also its conjugate $\overline{\mathcal{R}}_{i}$ representation of $\mathcal{G} \times \mathcal{F}$. As before, when $N$ is odd, we keep $N$ copies of Fermi multiplets in the $\mathcal{R}_{i}$ representation. There is no gauge invariant $E$ or $J$ type interaction we can write.

Since $2 \mathrm{~d}$ theories we obtain are chiral, we should worry about the potential gauge anomalies. When the gauge group $\mathcal{G}$ has no $\mathrm{U}(1)$ factor, we simply need to compute the trace anomaly $\operatorname{Tr}\left(\gamma^{3} \mathcal{G} \mathcal{G}\right)$. For simplicity, let us assume $\mathcal{G}$ is simple, and write the representation of the hypermultiplets to be $\left(\mathcal{R}_{\mathcal{G}, i}, \mathcal{R}_{\mathcal{F}, i}\right)$ where the first and second factor denotes that of gauge group and flavor group respectively. Then the gauge anomaly can 
be written as

$$
\operatorname{Tr}\left(\gamma^{3} \mathcal{G} \mathcal{G}\right)=(-1) h_{\mathcal{G}}^{\vee}+(N+1) h_{\mathcal{G}}^{\vee}-N \sum_{i} \operatorname{dim}\left(\mathcal{R}_{\mathcal{F}, i}\right) c_{2}\left(\mathcal{R}_{\mathcal{G}, i}\right)
$$

where $h^{\vee}$ is the dual coxeter number of the gauge group and $c_{2}$ is the index of the representation. Here the first and second term comes from the gauge multiplet and the adjoint chiral or Fermi. The last term comes from the matter multiplets. Note that the expression works for both signs of $N$. This expression is nothing but $N$ times the beta function of the $4 \mathrm{~d} \mathcal{N}=2$ theory before dimensional reduction. As long as we start from a $4 \mathrm{~d}$ conformal theory (which is crucial to perform $\mathrm{U}(1)_{r}$ twist), gauge anomalies are absent in $2 \mathrm{~d}$. As we have seen in section 4.2 .3 , this condition is tantamount to the modularity condition of the integrand of the specialized $4 \mathrm{~d}$ index $\mathcal{I}_{N}$.

\subsection{Central charge}

The central charge of the $2 \mathrm{~d} \mathcal{N}=(0,2)$ gauge theory can be computed easily from the 't Hooft anomalies of the R-symmetry as

$$
c_{R}=3 \operatorname{Tr}\left(\gamma^{3} R^{2}\right), \quad c_{R}-c_{L}=\operatorname{Tr} \gamma^{3} .
$$

Once we know the $R$-charge of the $2 \mathrm{~d}$ multiplets, we can compute the $2 \mathrm{~d}$ central charges.

From the $2 \mathrm{~d}$ viewpoint, there is no fixed choice of $R$-charge in general, because the theory has extra global symmetry (originating from the $\mathrm{U}(1)$ isometry of the $S^{2}$ ) that can be mixed with $R$-symmetry. For even $N<0$, one can assign $\mathrm{U}(1)_{R}$ charge for the $\Theta$ to be $1-2 \alpha$ and $Q, \tilde{Q}$ to be $\alpha$ for arbitrary $\alpha$. One canonical choice is $\alpha=0$. This is because there is a classical moduli space parametrized by $(Q, \tilde{Q})$ and in the semi-classical regime, where the field value is large, we expect there is a sigma-model description parametrized by the chiral multiplets [29]. When $N=-2$, this is the one studied in [26]. This choice gives central charges

$$
c_{L}^{0}=-2 N\left(n_{h}-n_{v}\right), \quad c_{R}^{0}=-3 N\left(n_{h}-n_{v}\right),
$$

which are positive for $N<0$, but negative for $N>0$.

When $N>0$, the canonical choice is to pick the $R$-charges for the chiral multiplets coming from the vector multiplets to be 0 , and those for the Fermi multiplets coming from the hypermultiplet to be 0 . We get

$$
c_{L}^{1}=N\left(n_{h}+2 n_{v}\right), \quad c_{R}^{1}=3 N n_{v} .
$$

This choice realizes the CFT on the Higgs branch parametrized by the scalar component of $\Phi$ field.

We find that the value $c_{L}$ gives the correct effective central charge $c_{\text {eff }}$ of $\operatorname{Tr} \mathcal{M}(q)^{N}=$ $\mathcal{I}_{N}(q)$

$$
c_{2 d, N}=N\left(n_{h}+2 n_{v}\right),
$$


for every value of $N$. For any $4 \mathrm{~d} \mathcal{N}=2 \mathrm{SCFT}$, one can define effective number of hypermultiplets and vector multiplets by extrapolating the relation between conformal anomalies $\left(a^{4 d}, c^{4 d}\right)$ and $\left(n_{h}, n_{v}\right)$ for Lagrangian theories

$$
n_{v}=4\left(2 a^{4 d}-c^{4 d}\right), \quad n_{h}=4\left(5 c^{4 d}-4 a^{4 d}\right) .
$$

We can write the $2 \mathrm{~d}$ central charges in terms of the $4 \mathrm{~d}$ conformal anomalies as

$$
c_{2 d, N}=12 N c^{4 d} .
$$

We note that when $N=-1$, the central charge for the left-movers is the same as the one given in [8]. For negative $N$, this value of central charge can be formally obtained by choosing the 'wrong R-charge' $R[Q]=1, R[\Theta]=0$ for the matter multiplets. This is related to the fact that the integrand of the elliptic genus with the 'wrong R-charge' assignment (for $N<0$ ) can be obtained from the specialization limit of the $4 \mathrm{~d}$ index $\mathcal{I}_{N}$. We discuss it in more detail in the next subsection.

\section{$5.3 \quad T^{2} \times S^{2}$ vs. $S^{1} \times S^{3}$ partition functions}

Let us discuss the connection between our setup $S^{1} \times S^{3}$ vs. $T^{2} \times S^{2}$. The partition function of a $2 \mathrm{~d} \mathcal{N}=(0,2)$ gauge theory on $T^{2}$, or elliptic genus, has been computed in [30-32]. The entire computation boils down to the integral over the moduli space of $\mathcal{G}$-flat connections $\mathcal{M}_{\text {flat }}\left(T^{2}, \mathcal{G}\right)$ on $T^{2}$ with 1-loop determinant

$$
Z_{\text {ell }}=\operatorname{Tr}(-1)^{F} q^{H_{L}} x^{F}=\int_{\mathcal{M}_{\text {flat }}\left(T^{2}, \mathcal{G}\right)} \prod_{i} d u_{i} d \bar{u}_{i} Z_{1-\text { loop }}(u, \bar{u})
$$

where $\mathcal{G}$ is the gauge group and $u, \bar{u} \in \mathcal{M}_{\text {flat }}\left(T^{2}, \mathcal{G}\right)$. The integrand has a number of poles, that are functions of various chemical potentials related to the global symmetry of the theory. By applying Stokes theorem carefully, the integral can be written in terms of a contour integral over certain holomorphic cycle of $\mathcal{M}_{\text {flat }}$. This yields so-called the Jeffrey-Kirwan residue formula

$$
Z_{\text {ell }}=\oint_{\mathrm{JK}} \frac{d z}{z} Z_{1-\mathrm{loop}}(z)
$$

where the integrand is now holomorphic in $z=e^{2 \pi i u}$. The integrand has to be elliptic (invariant under $z \rightarrow q z$ ) in order to be free of gauge anomalies.

The integrand for a $(0,2)$ chiral multiplet and a Fermi multiplet are given by

$$
Z_{\text {chiral }}(z ; q)=\frac{(q)_{\infty}}{\theta\left(q^{\frac{R}{2}} z ; q\right)}, \quad Z_{\mathrm{Fermi}}(z ; q)=\frac{\theta\left(q^{\frac{R+1}{2}} z ; q\right)}{(q)_{\infty}}
$$

where $R$ is the R-charge of the multiplet and $\theta(z ; q)$ is the theta function defined in equation (4.12) and $z$ is the fugacity for the $\mathrm{U}(1)$ flavor associated to the matter multiplet. Here we used the integrand for the NS sector elliptic genus, which agrees with the RR elliptic 
genus under the spectral flow and multiplicative factor. The NS elliptic genus is more convenient to compare with the $4 \mathrm{~d}$ index as we will see. For a vector multiplet, we have

$$
Z_{\mathrm{vec}}(z ; q)=(q)_{\infty}^{2 r} \prod_{\alpha \in \Delta^{ \pm}(\mathcal{G})} \vartheta\left(z^{\alpha} ; q\right)
$$

where $r$ is the rank of the gauge group $\mathcal{G}$ and $\Delta^{ \pm}(\mathcal{G})$ is the set of all (non-zero) roots of $\mathcal{G}$. in eq. (5.13) we use the short-hand notations $z^{\alpha} \equiv \prod_{i} z_{i}^{\alpha_{i}}$ and

$$
\vartheta(z ; q)=\theta(z ; q) /(q)_{\infty}
$$

Now, we write the elliptic genus of the $\mathcal{N}=(0,2)$ theory obtained from $4 \mathrm{~d} \mathcal{N}=2$ theory on $S^{2}$ with the twist of (5.1). Under the twisting and dimensional reduction, $4 \mathrm{~d}$ vector multiplet gives

$$
Z_{N>0}^{V}=\frac{(q ; q)^{2 r}}{\vartheta\left(v q^{\frac{R_{\Phi}}{2}} ; q\right)^{r(N+1)}} \prod_{\alpha \in \Delta^{ \pm}(\mathcal{G})} \frac{\vartheta\left(z^{\alpha} ; q\right)}{\vartheta\left(v q^{\frac{R_{\Phi}}{2}} z^{\alpha} ; q\right)^{N+1}} .
$$

The terms in the numerator come from the $\mathcal{N}=(0,2)$ vector and the denominator is coming from the chiral multiplets $\Phi$. Here $v$ is introduced to regulate the zero modes of the chiral/Fermi multiplets. It is the fugacity of the U(1) global symmetry associated to the rotation along $S^{2}$ direction. This is necessary because the theory is non-compact. As we have argued in the previous subsection, there is a CFT in the Higgs branch parametrized by $\Phi$. The correct R-charge for this case is $R_{\Phi}=0$.

When $N<0$, we get

$$
Z_{N<0}^{V}=(q ; q)^{2 r} \vartheta\left(v q^{\frac{R_{\Theta}+1}{2}} ; q\right)^{2 r(|N|-1)} \prod_{\alpha \in \Delta^{ \pm}(\mathcal{G})} \vartheta\left(z^{\alpha} ; q\right) \vartheta\left(v q^{\frac{R_{\Theta}+1}{2}} z^{\alpha} ; q\right)^{|N|-1}
$$

coming from the vector multiplet and adjoint Fermi multiplets $\Theta$.

When we choose the R-charge for the adjoint chiral $R_{\Phi}$ to be 0 and adjoint Fermi $R_{\Theta}$ to be -1 , we get a formula

$$
Z_{N}^{V}=\frac{(q ; q)^{2 r}}{\vartheta(v ; q)^{r(N+1)}} \prod_{\alpha \in \Delta^{ \pm}(\mathcal{G})} \frac{\vartheta\left(z^{\alpha} ; q\right)}{\vartheta\left(v z^{\alpha} ; q\right)^{N+1}}
$$

which works for all $N$. This choice of R-charge $R_{\Phi}=0$ for $N>0$ is physical and gives the elliptic genus of a CFT on the Higgs branch parametrized by the scalar component of $\Phi$. On the other hand, the choice of $R_{\Theta}=-1$ for $N<-1$ seems to be unphysical. But we find that the equation (5.17) is exactly the same one we get from the $S^{1} \times S^{3}$ partition function (superconformal index) upon the specialization limit $t=q p^{N+1}$ (when Haar measure is also included in the vector multiplet index) $\mathcal{I}_{N}^{V}$, with $v=1$.

For the hypermultiplet, we get

$$
Z_{N>0}^{H}=\vartheta\left(q^{\frac{R_{\Gamma}+1}{2}} z ; q\right)^{N}, \quad Z_{N<0}^{H}=\vartheta\left(q^{\frac{R_{Q}}{2}} ; q\right)^{-|N|},
$$


which comes from $|N|$ copies of Fermi or chiral multiplets. The physical choice is to set $R_{\Gamma}=0$ for $N>0$ and $R_{Q}=0$ for $N<0$, which gives the CFT on the Higgs branch. On the other hand, if we pick $R_{\Gamma}=0$ while $R_{Q}=1$, we get

$$
Z_{N}^{H}=\vartheta\left(q^{\frac{1}{2}} z ; q\right)^{N}
$$

Note that, this formula is valid regardless of whether $N$ is positive or negative. As before, choosing 'unphysical' R-charge assignment for $N<0$ gives the integrand to be the same as the specialization limit of the $4 \mathrm{~d}$ superconformal index $\mathcal{I}_{N}^{H}$.

With the integrand given as above, we need to evaluate Jeffrey-Kirwan residues to obtain the partition function on $T^{2} \times S^{2}{ }^{8}$ We note that the integrand (5.17) and (5.19) is exactly the same one we get from the $S^{1} \times S^{3}$ partition function upon specialization $t=q p^{N+1}$ :

$$
Z\left(T^{2} \times S^{2}\right)=\oint_{\mathrm{JK}} \frac{d z}{z} Z_{1-\mathrm{loop}}^{(N)},\left.\quad Z\left(S^{1} \times S^{3}\right)\right|_{t=q p^{N+1}}=\oint_{|z|=1} \frac{d z}{z} Z_{1-\mathrm{loop}}^{(N)}
$$

The crucial difference here is that unlike the case of $T^{2} \times S^{2}$, the integration contour is simply given by a unit circle. This integration contour is coming from the $\mathcal{G}$-flat connections on a circle $S^{1}$ instead of a torus $T^{2}$. One can consider both geometries as locally the same $T^{2} \times S^{2}$, where in one case $S^{1} \subset T^{2}$ is fibered over $S^{2}$ non-trivially. This fibration removes one of the circle directions in the integral, so that we get an integration over the flat connections on a circle. Another difference is that the $T^{2} \times S^{2}$ partition function is guaranteed to be modular (Jacobi form of weight 0) whenever the underlying theory is conformal, whereas the $S^{1} \times S^{3}$ partition function is not invariant, as we discussed in section 4.2.3.

Let us remark that when $N>0$, the above partition function $Z\left(T^{2} \times S^{2}\right)$, using the integrand (5.17) and (5.19), gives a valid elliptic genus of the $2 \mathrm{~d} \mathcal{N}=(0,2)$ CFT on the Higgs branch (along with the vector bundle) of the $2 \mathrm{~d}$ theory (which is not the same as the Higgs branch of $4 \mathrm{~d}$ theory) with central charge given in (5.9). In order to actually evaluate the integral, we need to consider fully refined fugacities for the extra global symmetry rotating the $N+1$ copies of the adjoint chiral multiplets, otherwise the elliptic genus diverge. This is the case because our target space is non-compact. For $N<0$, we should use the integrand (5.16) and (5.18) with $R_{Q}=0, R_{\Theta}=1$ and with all the flavor fugacities turned on to get a proper elliptic genus on the Higgs branch (with vector bundle). This is reminiscent of the fact that in the specializations of the index we had to insert operators to absorb fermionic/bosonic zero modes (for $N \neq-1$ ).

\footnotetext{
${ }^{8}$ Precisely speaking, we also need to sum over different flux sectors of the gauge fields on $S^{2}$ [33], which will yield multiple copies of the $2 \mathrm{~d}$ theory with different charges. Here we only focus on the zero flux sector.
} 


\section{BPS monodromy and the specialized index}

The quantum Kontsevich-Soibelman (KS) wall-crossing formula for $4 \mathrm{~d} \mathcal{N}=2$ field theories states $^{9}$ that the phase-ordered product ${ }^{10}[5]$

$$
M(q)=\prod_{\substack{\text { massive } \\ \text { BPS states }}}^{\circlearrowright}\left( \pm q^{s+1 / 2} X_{\gamma} ; q\right)_{\infty}^{(-1)^{2 s}}
$$

taken over the full spectrum of massive BPS states (of charge $\gamma \in \Gamma$ and spin $s$ ) in the clockwise order $\circlearrowright$ with respect to the BPS phase ${ }^{11} \arg Z_{\gamma}$, is independent of the particular BPS chamber up to conjugacy in the quantum torus algebra $\mathbb{T}$ with multiplication table

$$
X_{\gamma} X_{\gamma^{\prime}}=q^{\left\langle\gamma, \gamma^{\prime}\right\rangle / 2} X_{\gamma+\gamma^{\prime}} \quad \gamma, \gamma^{\prime} \in \Gamma .
$$

Hence the traces $\operatorname{Tr} M(q)^{N}$ are absolute wall-crossing invariants for all $N \in \mathbb{Z}$ and $q \in \mathbb{C}$ for which they are well defined.

Suppose we start from a UV SCFT $\mathcal{T}$, and mass-deform it in a generic way by going to the Coulomb branch. In the IR we end up with a theory $\mathcal{T}_{\text {IR }}$ which contains $r$ massless photons ( $r$ being the dimension of the Coulomb branch) and a non-trivial massive BPS spectrum. From this spectrum we compute the set of traces $\left\{\operatorname{Tr} M(q)^{N}\right\}$ which are well defined for $\mathcal{T}_{\text {IR }}$. The Kontsevich-Soibelman theory implies that the $\left\{\operatorname{Tr} M(q)^{N}\right\}$ depend only on the UV fixed point $\mathcal{T}$ and not on the particular mass-deformation $\mathcal{T}_{\text {IR }}$. Hence the $\left\{\operatorname{Tr} M(q)^{N}\right\}$ are to be thought of as invariant properties of the UV fixed point, in perfect analogy with the $2 \mathrm{~d}(2,2)$ situation $[1,4]$ reviewed in section 2 . The monodromy traces $\left\{\operatorname{Tr} M(q)^{N}\right\}$ then should be related to the natural SCFT invariants of $\mathcal{T}$, in particular to its specialized indices $\mathcal{I}_{N}(q)$. In order to connect the monodromy traces and usual SCFT invariants, one has to treat all BPS states of $\mathcal{T}_{\mathrm{IR}}$ on the same footing, including the massless ones which are not taken into account in the KS product $M(q)$. This gives an explanation of the prescription suggested in [9]. Then we redefine the monodromy operator by inserting the factors which correspond to the $r$ massless photons, seen as chargeless BPS vector multiplets,

$$
\mathcal{M}(q)=\frac{1}{(q)_{\infty}^{2 r}} M(q)
$$

and take the objects $\left\{\operatorname{Tr} \mathcal{M}(q)^{N}\right\}$ (to the extent such quantities are well defined) as the natural invariants to compare with the SCFT ones

$$
\left\{\operatorname{Tr} \mathcal{M}(q)^{N}\right\} \stackrel{?}{\longleftrightarrow}\left\{\mathcal{I}_{N}(q)\right\} .
$$

For $N=-1$ this map is expected to be especially simple: $\operatorname{Tr} \mathcal{M}(q)^{-1}$ gets identified with the SCFT index in the Schur limit which is independent of $p$. Strong evidence for this

\footnotetext{
${ }^{9}$ This version of the quantum KS formula holds under two genericity assumptions: 1) the only massless states are IR-free photons, and 2) massive BPS states with equal BPS phases, $\arg Z_{\gamma}=\arg Z_{\gamma^{\prime}}$, are mutually local i.e. $\left\langle\gamma, \gamma^{\prime}\right\rangle=0$.

${ }^{10}$ The sign \pm in the argument of the $q$-Pochammer symbol stands for the quadratic refinement of [34].

${ }^{11}$ Here $Z_{\gamma}$ is the $\mathcal{N}=2$ SUSY central charge associated to the charge $\gamma$; one has $Z_{\gamma+\gamma^{\prime}}=Z_{\gamma}+Z_{\gamma^{\prime}}$.
} 
identification was given in [9]. However, even for the Schur case we encounter a difficulty because the $\operatorname{Tr} \mathcal{M}(q)^{-1}$ is not always well defined, as computed from the BPS spectrum. For example for $\mathrm{SU}(2)$ with $N_{f}=4$, even if one uses the chamber where there are only a finite number of BPS particles, it turns out that the naive computation of $\operatorname{Tr} \mathcal{M}(q)^{-1}$ leads to divergencies which need to be regularized. We do not know of any general prescription for how to do this. In the absence of a general such prescription to regularize such computations we instead resort to cases which is free from these ambiguities as well as some general properties which do not depend on how the regularization is performed. There is an additional issue for $\operatorname{Tr} \mathcal{M}(q)^{N}$ for $N \neq-1$, because as we have explained we need to absorb extra zero modes to get a finite non-vanishing answer. Therefore the comparison of the corresponding computation with the UV computation becomes more difficult due to mapping operators from UV to IR. Nevertheless the general properties which are regularization independent (such as the effective $2 \mathrm{~d}$ central charge) as well as the example of Argyres-Douglas theories for which the characters seem to describe natural $2 \mathrm{~d}$ chiral algebras, support the picture we have found in the previous sections.

For the case of Lagrangian theories we have stronger results. First we show that the computation of the specialized index and the monodromy computation are identical for free theories (see also [9]). Moreover for interacting theories since the computation is independent of where we are in the moduli space, we can take the limit of $g_{Y M} \rightarrow 0$. In this limit the BPS spectrum becomes that of the free fields in the Lagrangian plus an infinite tower of dyonic BPS states whose mass $m \rightarrow \infty$. Thus the decoupling arguments for physical computations would lead us to consider the collection only of electric states and project to the gauge invariant subsector. This is identical to what one does both in the index computation and the Tr computation of the BPS particles and so the computations are identical. ${ }^{12}$ The only point which is not entirely trivial is to argue the more or less 'obvious' physical fact that infinitely massive states should decouple from the computation. An explicit proof of this turns out to be difficult because of the ambiguities noted above in defining the trace. Instead we resort to highly non-trivial consistency checks that shows we need to set the product of the contributions of BPS states coming from the dyonic towers to 1, i.e. we can ignore them in the extreme weak coupling limit.

The organization of this section is as follows: we first talk about the free case and then discuss in detail the argument why the tower of dyonic states should not contribute. We then talk about some general aspects of the trace of the monodromy which applies to all theories, and not just the Lagrangian ones. In the following section we show how these general expectations are borne out in the context of Argyres-Douglas theories.

\subsection{Free hypers and hypers coupled to abelian gauge groups}

There is a case in which the map (6.4) is obvious, namely when the BPS particles are all mutually local. Then the theory has a unique BPS chamber, and all BPS factors in the KS product belong to a commutative sub-algebra of the quantum torus algebra $\mathbb{T}$. We may think of $\mathcal{M}(q)^{N}$ as ordinary functions of the fugacities for the local Noether charges

\footnotetext{
${ }^{12}$ This argument also extends to the case of insertion of line operators.
} 
(electric and flavor). The traces $\left\{\operatorname{Tr} \mathcal{M}(q)^{N}\right\}$ are then the integrals of these functions with respect to the electric fugacities.

Examples of this situation are the free theories of hypermultiplets and vector multiplets, as well as the Abelian gauge theories coupled to hypermultiplets considered in section 4.3.1. (These last theories are however not UV complete, so our arguments should be considered purely formal in this case).

In the free theory case, each BPS factor in the KS product (6.1) is equal to the inverse of the corresponding factor in the Schur index, and $\mathcal{M}(q)^{N}$ (which in the free case coincides with its trace) is by definition equal to the index $\mathcal{I}_{N}$ described in section 4.1.2.

In the SQED case, $\mathcal{M}(q)^{N}$, as a function, is by construction equal to the integrand of the specialized index $\mathcal{I}_{N}$ (cfr. section 4.3.1), while taking their trace amounts to integrating along the unit circle all the non-flavor fugacities (cfr. equation (6.71)). Since the integrand depends only on the electric and flavor fugacities, this reproduces the same prescription we gave in section 4.3.1 to compute the specialized index $\mathcal{I}_{N}$. For instance, for SQED with one quark we have

$$
\begin{aligned}
\operatorname{Tr} \mathcal{M}(q)^{N} & =(q)_{\infty}^{2} \operatorname{Tr}\left(\prod_{n=0}^{\infty}\left(1-q^{n+1 / 2} X\right)^{N}\left(1-q^{n+1 / 2} X^{-1}\right)^{N}\right) \\
& \equiv(q)_{\infty}^{2} \oint \frac{d z}{2 \pi i z}\left(1-q^{n+1 / 2} z\right)^{N}\left(1-q^{n+1 / 2} z^{-1}\right)^{N}=\mathcal{I}_{N}(q)
\end{aligned}
$$

Note here that the comparison between the trace computation and index computation involves the integral representation of the monodromy trace.

These simple examples corroborate the idea of a direct identification in (6.4).

\subsection{Physical arguments for models having Lagrangian formulation}

The next class of models is the $\mathcal{N}=2$ non-Abelian gauge theories coupled to hypermultiplets, which coincide with the $\mathcal{N}=2$ models with a Lagrangian description.

From the superconformal index side, the situation looks rather similar to the SQED one. The specialized indices are expressed as integrals over the electric fugacities dual to the Cartan charges of the non-Abelian electric gauge group (with the appropriate gauge invariant measure), while the integrand is a product of factors associated to the quarks states, which have the same form as in the Abelian case, and new factors for the charged $W$ bosons which are essentially identical to the KS monodromy factors for BPS vector multiplets with the $W$ boson quantum numbers, see equation (6.1).

From the quantum monodromy side the situation looks far more complicated. In any chamber where the Yang-Mills coupling $g_{\mathrm{YM}}$ is small, the BPS spectrum of $\mathcal{N}=2$ supersymmetric quantum chromodynamics (SQCD) contains two kinds of states:

a) the perturbative spectrum, consisting of mutually-local particles with zero magnetic charges, whose masses remain bounded as $g_{\mathrm{YM}} \rightarrow 0$. There are only finitely many such light particles, and they have spin $\leq 1$, so belong to either hypermultiplets (quarks) or vector multiplets (photons and charged $W$ bosons); 
b) infinite towers of heavy dyonic states, with non-zero magnetic charges, whose masses are of order $1 / g_{\mathrm{YM}}^{2}$ as $g_{\mathrm{YM}} \rightarrow 0$ and whose spins are not necessarily bounded.

Thus, in a weakly coupled chamber, the KS product (6.1) contains the factors

$$
\left( \pm q^{s+1 / 2} X_{\gamma} ; q\right)_{\infty}^{(-1)^{2 s}}
$$

of all these infinitely many BPS states. The KS factors associated to the light states a) are essentially equal to the corresponding factors in the specialized index integrand; but in addition we have infinitely many other factors associated to the heavy dyons. Since taking the trace is the same as integrating over the non-flavor fugacities, up to possible subtleties with the integration measure, the only difference between the definitions of the superconformal index $\mathcal{I}_{N}(q)$ and $\operatorname{Tr} \mathcal{M}(q)^{N}$ is the insertion in the latter of the factors associated to the heavy dyonic towers. Were we allowed to forget these dyonic factors, the two sets of SCFT invariants in equation (6.4) would be equal on the nose, as they are for free theories and SQED.

The idea that at weak coupling we may simply neglect the dyonic factors sounds physically plausible. Indeed, the monodromy traces are independent of the Yang-Mills coupling $g_{\mathrm{YM}}$, and they may be safely computed at parametrically small $g_{\mathrm{YM}}$. In this limit the dyon masses go to infinity; physical intuition says that they decouple completely. Hence, at least heuristically, at extreme weak coupling we may identify $\operatorname{Tr} \mathcal{M}(q)^{N}$ with the corresponding specialized index for all $\mathcal{N}=2$ models having a weakly coupled Lagrangian description.

The $\left\{\operatorname{Tr} \mathcal{M}(q)^{N}\right\}$ are chamber independent (as long as they are well defined). Many interesting $\mathcal{N}=2$ non-Abelian gauge theories have strongly-coupled chambers with finite BPS spectra consisting of just $h$ hypermultiplets. In this situation $\left\{\operatorname{Tr} \mathcal{M}(q)^{N}\right\}$ may be computed directly from the strongly coupled phase. In this way one may check the above physical picture at extreme weak coupling against an independent computation of the monodromy traces. Such a check was performed in [9] for SQCD with gauge group $\mathrm{SU}(2)$ and $N_{f} \leq 3$ : it was shown that, for these models, $\operatorname{Tr} \mathcal{M}(q)^{-1}$ as computed from the minimal BPS chamber with $h=N_{f}+2$ agrees with the extreme weak coupling answer, i.e. with its computation at weak coupling neglecting the dyons. For instance, for pure $\mathrm{SU}(2)$ SYM $\operatorname{Tr} \mathcal{M}(q)^{-1}$, computed as an integral over the electric and magnetic fugacities with all dyonic factors inserted in the integrand, is (appendix C),

$$
\operatorname{Tr} \mathcal{M}(q)^{-1}=\int \frac{d \theta d \phi}{(2 \pi)^{2}}\left|\frac{(q)_{\infty}\left(q e^{i(\theta+\phi)}\right)_{\infty}}{\left(q^{1 / 2} e^{i \theta}\right)_{\infty}\left(q^{1 / 2} e^{i \phi}\right)_{\infty}}\right|^{2}=\psi\left(1, q^{2}\right)
$$

which agrees with the Schur index (4.81) given by the corresponding integral without the dyonic contributions [9]. The case $N=-1$ is especially simple since there is no subtlety with the integration measure (involving the absorbtion of bosonic/fermionic zero modes). Indeed, consider $\mathcal{N}=2 \mathrm{SQCD}$ with gauge group $\mathcal{G}$ of rank $r_{\mathcal{G}}$ and let $\Delta^{+}$be the set of its positive roots. As a function of the electric fugacities $e^{i H}, H \in$ (Cartan subalgebra), the 
light BPS factors in the KS product $\mathcal{M}(q)^{-1}$ are

$$
\begin{aligned}
(q)_{\infty}^{2 r_{\mathcal{G}}}\left(\prod_{\alpha \in \Delta^{+}}\left(q e^{i \alpha(H)} ; q\right)_{\infty}\left(e^{i \alpha(H)} ; q\right)_{\infty}\left(e^{-i \alpha(H)}\right)_{\infty}\left(q e^{-i \alpha(H)}\right)_{\infty}\right)(\text { quark factors }) \equiv \\
\quad \overbrace{\left(\prod_{\alpha \in \Delta^{+}}\left(1-e^{i \alpha(H)}\right)\left(1-e^{-i \alpha(H)}\right)\right)}^{\text {Weyl measure }} \operatorname{PE}\left[-\frac{2 q}{1-q} \chi_{\text {adj }}(H)\right] \text { (quark factors) },
\end{aligned}
$$

so that the $W$ boson KS factors correctly reproduce the gauge invariant Weyl measure as well as the proper factor from the vectors fields in the Schur index integrand.

Ignoring the dyonic towers - if legitimate - would be a major simplification for the computation of monodromy traces of $\mathcal{N}=2$ models with a Lagrangian formulation and would make their equivalence obvious. In view of this, it is interesting to see to what extend this procedure may be justified. In the next couple of subsections we argue why the natural regularization of the contribution of the infinite tower of dyonic states should set their contribution to the monodromy operator to 1 . In particular we show that setting their contribution to 1 leads to the action of monodromy operator on the line operator which is consistent with what one expects that action of the monodromy operator be on the line operators from other viewpoints.

\subsubsection{The physical picture at extreme weak coupling}

Let us make the physical picture more precise. For $q \sim 1$ the quantum torus becomes classical and the $\left\{X_{\gamma}\right\}$ may be identified with the Darboux coordinates $\left\{\mathcal{X}_{\gamma}\right\}$ on the hyperKähler target space of the $\sigma$-model dual to the $3 \mathrm{~d}$ compactification of the $4 \mathrm{~d} \mathcal{N}=2$ theory [34]. In other words, the $\left\{X_{\gamma}\right\}$ and the $\left\{\mathcal{X}_{\gamma}\right\}$ have the same transformation under the monodromy as $\tau \rightarrow 0$. The $\mathcal{X}_{\gamma}$ has the physical interpretation of expectation value of the supersymmetric Wilson-'t Hooft line operator of electro-magnetic charge $\gamma$. In a given quantum phase of the non-Abelian gauge theory, one distinguishes two classes of such line operators, "light" and "heavy" [35-37]. "Heavy" lines may mix with "light" ones, but "light" ones may only mix between themselves: this is just a rephrasing in the physical language of the mathematics of the Stokes phenomenon for the Darboux coordinates $\mathcal{X}_{\gamma}$ [34]. In the Coulomb phase, the "light" lines are the electric ones while the dyonic lines are "heavy". It follows that under monodromy the electric lines go into themselves while the magnetic ones may mix with the electric ones. The mixing is dictated by the Witten effect [38]. In the $\mathcal{N}=2$ case these general non-perturbative arguments may be made more explicit as we are going to discuss.

SU(2) SQCD with $N_{f}$ quarks. For definiteness, we consider the case of $\mathcal{N}=2$ SQCD with gauge group $\mathrm{SU}(2)$ and $N_{f}$ flavors of quark [3]; the general Lagrangian case is similar. Since the $\mathrm{U}(1)_{R}$ symmetry is anomalous, a $\mathrm{U}(1)_{R}$ rotation by $2 \pi$ is equivalent to a shift of the Yang-Mills angle $\theta$ by a multiple of $2 \pi$; then the effect of the monodromy on the electric $e$ and magnetic charge $m$ is

$$
(e, m) \longrightarrow\left(e+2\left(4-N_{f}\right), m\right),
$$


that is, in the weakly coupled phase the electric and magnetic lines transform under monodromy as

$$
\mathcal{X}_{e} \rightarrow \mathcal{X}_{e}, \quad \mathcal{X}_{m} \rightarrow \mathcal{X}_{m+2\left(4-N_{f}\right) e}
$$

Indeed, in general one has [34]

$$
\mathcal{X}_{\gamma}=\exp \left(R a_{\gamma} \zeta+i \theta_{\gamma}+R \bar{a}_{\gamma} / \zeta+\cdots\right)
$$

where $a_{\gamma} \equiv Z_{\gamma}$ and $\cdots$ stands for terms exponentially suppressed as $R \rightarrow \infty$. Since [3]

$$
a_{m} \approx-\frac{4-N_{f}}{\pi i} a_{e} \log \left(a_{e} / \Lambda\right), \text { for }\left|a_{e}\right| \ggg \Lambda,
$$

while the monodromy acts on the Lagrangian fields as $a_{e} \rightarrow e^{2 \pi i} a_{e}$, we have the expected result (6.12). Thus, as $q \rightarrow 1$ the action of the monodromy is simply

$$
X_{e} \rightarrow X_{e}, \quad X_{m} \rightarrow X_{m+2\left(4-N_{f}\right) e}
$$

How is the physical answer (6.12) related to the quantum monodromy $\mathcal{M}(q)$ written as the KS product of BPS factors (6.1)? To compare the two pictures of quantum monodromy, we choose the mass-deformations so that the BPS phases of the quark states are aligned with those of the $W$ boson (since $W$ bosons and quarks are mutually local, this is still a 'generic' situation according to our definition). Identifying BPS particles with representations of the corresponding BPS quiver ${ }^{13} Q$, and using the conventions which are standard in that context $[39,40]$, the BPS phase of the light BPS states ( $W$-bosons and quarks) is set to $\pi / 2$ while the phase of the monopole is 0 . Thus states whose central charge belong to the upper (lower) half-plane have positive (negative) electric charge, while states with central charges in the right (left) half-plane have positive (negative) magnetic charge. The monodromy $\mathcal{M}(q)$ computed from the chamber at strong coupling with finite number of BPS states is the monodromy which starts at phase zero (the monopole phase) makes a $2 \pi$ rotation and comes back to zero. Instead the monodromy $\mathcal{M}(q)^{\mathrm{el}}$ which preserves the light electric lines, eq. (6.12), starts and ends at $\pi / 2$. Thus, as operators,

$$
\mathcal{M}(q)^{\mathrm{el}}=K S(0, \pi / 2) \mathcal{M}(q) K S(0, \pi / 2)^{-1},
$$

where $K S(\alpha, \beta)$ is the KS ordered product (6.1) taken over BPS states with $\alpha \leq \arg Z_{\gamma}<\beta$. Thus the monodromy operators which are relevant at strong coupling and weak coupling are different operators in the same conjugacy class (recall that monodromy is defined only up to conjugacy). Their intertwinner, $K S(0,2 \pi)$, is a quite complicated object: it is an infinite product over the BPS dyonic towers with positive magnetic charge and non-negative electric charge. Relating monodromy computations at strong and weak coupling requires to give a definite meaning to the formal infinite non-commutative product $K S(0,2 \pi)$.

\footnotetext{
${ }^{13}$ In this paper when we refer to quiver, we mean the quiver quantum mechanics which captures the BPS spectrum of the $4 \mathrm{~d}$ theory and not the quiver theory describing the Lagrangian degrees of freedom of the $4 \mathrm{~d}$ gauge theory.
} 
From (6.12), we see that $\mathcal{M}(q)^{\mathrm{el}}$ commutes with $X_{e}$. Under a mild regularity assumption, one concludes that $\mathcal{M}(q)^{\mathrm{el}}$ does not contain $X_{m}$, i.e. that it is a function only of $X_{e}$ and the $\mathrm{SO}\left(2 N_{f}\right)$ flavor fugacities $y_{a}$

$$
\mathcal{M}(q)^{\mathrm{el}}=m\left(X_{e}, y_{a} ; q\right)
$$

The function $m\left(X_{e}, y_{a} ; q\right)$ is determined from its adjoint action on the magnetic lines, eq. (6.12),

$$
\mathcal{M}(q)^{\mathrm{el}} X_{m}\left(\mathcal{M}(q)^{\mathrm{el}}\right)^{-1}=X_{m+2\left(4-N_{f}\right) e} .
$$

If we know the singularity structure of the function $m\left(X_{e}, y_{a} ; q\right)$, we may use this equation to determine it up to multiplication by a $c$-number function of $q$ and $y_{a}$. In facts, eq. (6.15) translates into a functional equation for $m\left(X_{e}, y_{a} ; q\right)$ related to the Yang-Baxter equation studied in [41]. As a preparation, consider the $\theta$-function

$$
\theta(z ; q)=\sum_{n \in \mathbb{Z}} q^{n(n-1) / 2}(-z)^{n}=(q ; q)_{\infty}(z ; q)_{\infty}(q / z ; q)_{\infty}
$$

It satisfies the functional equation

$$
\theta(q z)=-z^{-1} \theta(z) \quad \Rightarrow \quad \theta\left(q^{2} z\right)=\left(q z^{2}\right)^{-1} \theta(z)
$$

Then for operators satisfying $X_{e} X_{m}=q X_{m} X_{e}$ we have [41]

$$
\theta\left(q^{a} X_{2 e} ; q\right)^{-1} X_{m} \theta\left(q^{a} X_{2 e} ; q\right)=X_{m} \theta\left(q^{a+2} X_{2 e} ; q\right)^{-1} X_{m} \theta\left(q^{a} X_{2 e} ; q\right)=q^{2 a+1} X_{m} X_{4 e}
$$

All singularities of the monodromy function $m\left(X_{e}, y_{a} ; q\right)$ should have a physical origin. The only possible mechanism to generate a singularity in the monodromy is that some generically massive BPS particles become massless, which implies a discontinuity in the KS product over massive BPS states. Since in the weak coupling chamber the $W$ boson is nowhere massless, for pure $\mathrm{SU}(2)$ it is natural to look for a regular solution to (6.15). We compare the adjoint action (6.15) with the following "Yang-Baxter identity"

$$
\begin{gathered}
{\left[\theta\left(q^{a} X_{2 e} ; q\right) \theta\left(q^{b} X_{2 e} ; q\right)\right]^{-1} X_{m}\left[\theta\left(q^{a} X_{2 e} ; q\right) \theta\left(q^{b} X_{2 e} ; q\right)\right]=} \\
=q^{2(a+b)+2} X_{m} X_{8 e}=q^{2(a+b-1)} X_{m+8 e}
\end{gathered}
$$

Then the general regular solution to (6.12) is

$$
\begin{aligned}
\left(\mathcal{M}(q)^{\mathrm{el}}\right)^{-1} & =f(q) \theta\left(q X_{2 e} ; q\right) \theta\left(X_{2 e} ; q\right)= \\
& =f(q)(q ; q)_{\infty}^{2}\left(q X_{2 e} ; q\right)_{\infty}\left(X_{2 e} ; q\right)_{\infty}\left(q X_{2 e}^{-1} ; q\right)_{\infty}\left(X_{2 e}^{-1} ; q\right)_{\infty}
\end{aligned}
$$

with $f(q)$ undetermined. Choosing $f(q)=1$ we get the answer that we expected from the heuristic argument that infinitely heavy particles (the dyons at extreme weak coupling) have no effect on the physics. 
$\mathrm{SU}(2) S Q C D$ with $N_{f}$ quarks. The argument is easily generalized to $N_{f} \neq 0$. However in this case it is possible for the quarks to get massless while keeping $g_{\mathrm{YM}}$ parametrically small. Hence we expect the solution $m\left(X_{e}, y_{a} ; q\right)$ to $(6.15)$ to have singularities as a function of the quark fugacities $y_{a}$ of the appropriate form. Then, defining the theta function

$$
\tilde{\theta}(z ; q)=\sum_{n=0}^{\infty} q^{n^{2} / 2} z^{n}
$$

we solve the equation (6.15) in the form

$$
\left(\mathcal{M}(q)^{\mathrm{el}}\right)^{-1}=f\left(q ; y_{a}\right) \frac{\theta\left(q X_{2 e} ; q\right) \theta\left(X_{2 e} ; q\right)}{\prod_{i=1}^{N_{f}}\left(\tilde{\theta}\left(y_{a} X_{e} ; q\right) \tilde{\theta}\left(y_{a}^{-1} X_{e} ; q\right)\right)} .
$$

Setting the undetermined function $f\left(q ; y_{a}\right)=(q)_{\infty}^{2 N_{f}}$ we recover the physically expected answer. In particular, the form of the singularity in flavor fugacity is consistent with its physical interpretation in terms of BPS states becoming massless. Additional (and stronger) arguments for equation (6.22) are presented in the following subsections.

From this analysis ${ }^{14}$ we learn that the physical picture of the quantum monodromy at weak coupling where we simply forget the heavy dyons, and the strong coupling picture where $\mathcal{M}(q)$ is a complicated element of the quantum torus algebra depending non-trivially on both $X_{e}$ and $X_{m}$ are not in contradiction since the two monodromies are different elements in the same conjugacy class, eq. (6.13). Their intertwinner, $K S(0, \pi / 2)$, is a formal operator whose precise definition is hard to pinpoint. Different prescriptions may lead to different answers, so we must expect a degree of ambiguity in computations which require explicit use of the strong-weak intertwinner $K S(0, \pi / 2)$. We can interpret the results of [9], for the Schur case, as a confirmation of the general arguments presented here.

\subsubsection{Decoupling of infinitely massive dyons and S-duality}

The result of the physical argument in section 6.2.1, say equation (6.22) for SU(2) SQCD with $N_{f}$ flavors, implies strong (and somehow paradoxical) properties of the KS products in $\mathcal{N}=2$ non-Abelian gauge theories. First of all, the mathematical legitimacy of neglecting the heavy dyonic states in the KS product requires that the KS products over the dyons, as operators, satisfy remarkable identities. We shall check these identities in the next subsection.

In addition, the physical picture leads to apparent paradoxes, since at first sight it seems to clash with $S$-duality. Consider $\mathrm{SU}(2)$ with $N_{f}=4$. From the physical picture advocated above, it is natural to conclude that the monodromy has the form (6.22) with $N_{f}=4$ : this is the result of 'forgetting' the heavy dyons. But which 'heavy' dyons are we supposed to forget? The notion of a BPS state to be a dyon depends on the $S$-duality frame

\footnotetext{
${ }^{14}$ We warn the reader that the 'physical' argument suffers from a minor ambiguity. In facts, we deduced (6.15) from the $\tau \equiv \log q / 2 \pi i \rightarrow 0$ limit, and hence the actual answer may differ by a factor which is trivial in this limit. In practice, viewing the KS product as a partition function for BPS states, the answer may be off by finitely many modes. An example is given by eq. (6.7) where the monodromy differs from the index integrand by the zero-mode factors which produce the correct gauge invariant measure. This will not affect robust quantities like $c_{\text {eff }}$.
} 
we use, and its being 'heavy' or not depends on the value of the Yang-Mills coupling $g_{\mathrm{YM}}$. But the KS monodromy does not depend on $g_{\mathrm{YM}}$. Our physical picture would describe the monodromy in one $S$-frame as the function (6.22) of $X_{e}, \mathcal{M}(q)=m\left(X_{e}, y_{a} ; q\right)$, and in a different frame as the same function but of different arguments, $\mathcal{M}(q)=m\left(X_{p e+q m}, y_{a}^{\prime} ; q\right)$, (here $(p, q)$ are coprime integers). It seems we got a paradox.

The solution of the paradox is that the monodromy $\mathcal{M}(q)$ is not unique, only its conjugacy class is an absolute invariant. The physical picture is consistent provided the two candidate monodromies are conjugate by the appropriate KS operator i.e.

$$
m\left(X_{p e+q m}, y_{a}^{\prime}, q\right)=K S\left(\arg Z_{p e+q m}, \arg Z_{e}\right)^{-1} m\left(X_{e}, y_{a} ; q\right) K S\left(\arg Z_{p e+q m}, \arg Z_{e}\right),
$$

where $K S\left(\theta, \theta^{\prime}\right)$ is the KS product over all BPS states with charges $\gamma$ satisfying

$$
\theta^{\prime}<\arg Z_{\gamma}<\theta
$$

That $m\left(X_{p e+q m}, y_{a}^{\prime}, q\right)$ and $m\left(X_{e}, y_{a} ; q\right)$ are conjugate in the quantum torus algebra is obvious (at least when $y_{a} \equiv 1$ ); that the required action of $\mathrm{SL}(2, \mathbb{Z})$ is generated by the KS products in the appropriate angular sectors will be shown, for the $\mathrm{SU}(2) N_{f}=4$ example, in section 6.3 starting from the monodromy as computed from the strongly-coupled finite BPS chamber.

\subsubsection{Product identities for dyonic towers}

The 'physical' picture implies some remarkable identities for the KS products over dyonic towers. Here we focus on pure $\mathrm{SU}(2)$, but the results apply with minor modifications to all Lagrangian theories.

The notion of the quantum monodromy arises from considering a closed path in parameter space along which the phase of the central charge increases by $2 \pi$

$$
Z \rightarrow e^{2 \pi i t} Z \quad t \in[0,1] .
$$

At $t \rightarrow 1$ we go back to the original theory, but the line operators $\mathcal{X}_{\gamma}$ do not return to themselves. The map $\left\{\mathcal{X}_{\gamma}\right\}_{t=0} \rightarrow\left\{\mathcal{X}_{\gamma}\right\}_{t=1}$ defines the adjoint action of the quantum monodromy $\mathcal{M}(q)$. However, the theory has come back to itself already at $t=1 / 2$, since $\gamma \rightarrow-\gamma$ corresponds to the action of the $\mathrm{SU}(2)$ Weyl symmetry, which is part of the gauge group. Hence the argument of the previous subsection should hold also for the KS product of BPS states whose central charges belong to a half-plane. In our conventions that the BPS phase of the $W$-boson is $\pi / 2$, the previous argument would imply that the product over all BPS states with central charge in the positive half-plane, i.e. over all dyons with positive magnetic charge, is just 1

$$
\prod_{\substack{\text { BPS states with } \\-\pi / 2<\arg Z_{\gamma}<\pi / 2}}^{\curvearrowright}\left(q^{1 / 2} X_{\gamma} ; q\right)_{\infty}=1,
$$

or, explicitly,

$$
\prod_{j \in \mathbb{Z}}^{\leftarrow}\left(q^{1 / 2} X_{m+2 j e} ; q\right)_{\infty}=1
$$


where the notation $\overleftarrow{\prod}$ means that the factor $\left(q^{1 / 2} X_{m+2 j e} ; q\right)_{\infty}$ is on the left of the factors $\left(q^{1 / 2} X_{m+2 \ell e} ; q\right)_{\infty}$ with $\ell<j$, and $X_{m}, X_{2 e}$ satisfy the commutation relations $X_{2 e} X_{m}=$ $q^{2} X_{m} X_{2 e}$. Showing that the identity (6.26) holds (in the appropriate sense) is a important check on the full picture.

Formally, we may expand the inverse of the l.h.s. of (6.26) into terms of definite magnetic charge $M$

$$
\prod_{j \in \mathbb{Z}}^{\rightarrow}\left(q^{1 / 2} X_{m+2 j e} ; q\right)_{\infty}^{-1}=\sum_{M=0}^{\infty} X_{m}^{M} A_{M}\left(X_{2 e} ; q\right) .
$$

To write the $A_{M}\left(X_{2 e} ; q\right)$ in compact form, we introduce the following notation: for $M \in \mathbb{N}$, we write $\mathbb{A}(M)$ for the set of all maps $\boldsymbol{k}: \mathbb{Z} \rightarrow \mathbb{N}, j \mapsto k_{j}$, such that $\sum_{j} k_{j}=M$ (in particular, at most $M k_{j}$ 's are non zero). Then we put

$$
|\boldsymbol{k}|=\sum_{j \in \mathbb{Z}} k_{j}, \quad[\boldsymbol{k}]=\sum_{j \in \mathbb{Z}} j k_{j}, \quad(q)_{\boldsymbol{k}}=\prod_{j}(q)_{k_{j}},
$$

and consider the quadratic form

$$
Q(\boldsymbol{k})=\sum_{i, j} \min (i, j) k_{i} k_{j}
$$

which is an inverse of the $A_{\infty}^{\infty}$ Cartan matrix

$$
\sum_{j}\left(2 \delta_{i j}-\delta_{i, j-1}-\delta_{i, j+1}\right) \min (j, k)=\delta_{i, k}
$$

Then formally

$$
A_{M}\left(X_{2 e} ; q\right)=q^{M / 2} \sum_{\boldsymbol{k} \in \mathbb{A}(M)} \frac{q^{Q(\boldsymbol{k})}}{(q)_{\boldsymbol{k}}} X_{2 e}^{[\boldsymbol{k}]} .
$$

To give a concrete meaning to the operator in the 1.h.s. of (6.27), we have to make sense of the infinite sums $\left\{A_{M}\left(X_{2 e} ; q\right)\right\}_{M \in \mathbb{N}}$. For $M=0$ we have simply $A_{M}\left(X_{2 e} ; q\right)=1$. For $M>0$ we focus on the set of functional equations satisfied by the would be sums. The group $\mathbb{Z}$ acts on the set $\mathbb{A}(M)$ by the shift operators $[n]: \mathbb{A}(M) \rightarrow \mathbb{A}(M)$ given by $\boldsymbol{k}[n]_{j}=k_{j-n}$. Under the unit shift [1] we have

$$
|\boldsymbol{k}| \rightarrow|\boldsymbol{k}|, \quad[\boldsymbol{k}] \rightarrow[\boldsymbol{k}]+|\boldsymbol{k}|, \quad Q(\boldsymbol{k}) \rightarrow Q(\boldsymbol{k})+|\boldsymbol{k}|^{2}, \quad(q)_{\boldsymbol{k}} \rightarrow(q)_{\boldsymbol{k}}
$$

Summing over the shifted maps $\boldsymbol{k}[1] \in \mathbb{A}(M)$, we get

$$
\begin{aligned}
A_{M}\left(X_{2 e} ; q\right) & =q^{M / 2} \sum_{\boldsymbol{k}[1] \in \mathbb{A}(M)} \frac{q^{Q(\boldsymbol{k}[1])}}{(q)_{\boldsymbol{k}[1]}} X_{2 e}^{[\boldsymbol{k}[1]]}= \\
& =\left(q^{M^{2}} X_{2 e}^{M}\right) q^{M / 2} \sum_{\boldsymbol{k} \in \mathbb{A}(M)} \frac{q^{Q(\boldsymbol{k})}}{(q)_{\boldsymbol{k}}} X_{\delta}^{[\boldsymbol{k}]} \equiv q^{M^{2}} X_{2 e}^{M} A_{M}\left(X_{2 e} ; q\right),
\end{aligned}
$$

so that $A_{M}\left(X_{2 e} ; q\right)$ satisfies the functional equation

$$
\left(1-q^{M^{2}} X_{2 e}^{M}\right) A_{M}\left(X_{2 e} ; q\right)=0
$$


For $M>0$ the only regular solution to this equation is $A_{M}\left(X_{2 e} ; q\right)=0$. The regularity condition we use here reflects the regularity condition we used to pinpoint a unique solution for the Yang-Baxter equation of pure SU(2) in the previous subsection. Then we conclude that $A_{M}\left(X_{2 e} ; q\right)=\delta_{M, 0}$, that is

$$
\prod_{j \in \mathbb{Z}}^{\rightarrow}\left(q^{1 / 2} X_{m+2 j e} ; q\right)_{\infty}^{-1}=1,
$$

in agreement with the decoupling of infinitely massive dyons.

\subsection{SU(2) SQCD with $N_{f}=4$ and $S$-duality}

To further illustrate and corroborate the above physical picture of the quantum monodromy $\mathcal{M}(q)$, we present some non-trivial monodromy computations in SCFT examples.

The simplest non-trivial $\mathcal{N}=2$ SCFT with a Lagrangian description is SQCD with gauge group $\mathrm{SU}(2)$ and four fundamental quarks. We choose its BPS quiver in the form $[42-44]$

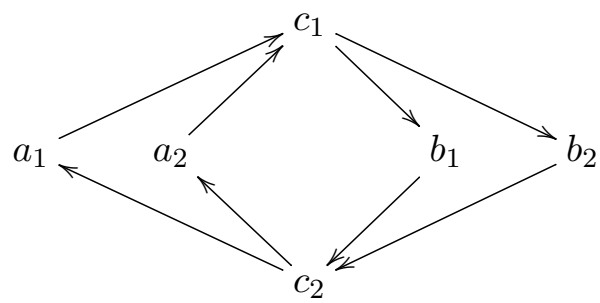

which has the $\mathbb{Z}_{2}$ symmetry $\iota$

$$
\left(a_{1}, a_{2}, c_{1}, b_{1}, b_{2}, c_{2}\right) \stackrel{\iota}{\longleftrightarrow}\left(b_{1}, b_{2}, c_{2}, a_{1}, a_{2}, c_{1}\right),
$$

which is a simple instance of Galois symmetry in the sense of [45] which, when present, is the most powerful tool to compute the quantum monodromy. Since $\iota$ is an involutive automorphism of the quiver, for all $\gamma, \gamma^{\prime} \in \Gamma$,

$$
\left\langle\iota(\gamma), \gamma^{\prime}\right\rangle=\left\langle\gamma, \iota\left(\gamma^{\prime}\right)\right\rangle \quad \Rightarrow \quad\langle\gamma, \iota(\gamma)\rangle=0
$$

i.e. BPS states in the same orbit of the Galois symmetry are automatically mutually-local.

$\mathrm{SU}(2)$ with $N_{f}=4$ has a strongly-coupled finite BPS chamber with 12 hypermultiplets [42-44], which is invariant under the $\mathbb{Z}_{2}$ symmetry $\iota$ in the sense that if $\gamma \in \Gamma$ is the charge of a stable hypermultiplet so is $\iota(\gamma)$. Indeed the set of the charge vectors of the 12 hypermultiplets is given by the union $\Delta^{+} \amalg \Delta^{+}$of the positive roots $\Delta^{+}$of the two $A_{3}$ Dynkin full subquivers over the nodes $\left\{a_{1}, a_{2}, c_{1}\right\}$ and $\left\{b_{1}, b_{2}, c_{2}\right\}$ which are interchanged by $\iota[42,44,45]$. Inside this strongly-coupled chamber there is a locus where the central charge is also $\iota$-symmetric, i.e. $Z_{\iota(\gamma)}=Z_{\gamma}$. Finally the quadratic refinement is also $\iota$-invariant. Then the monodromy KS product, as computed at the $\iota$-invariant locus in the strongly-coupled finite chamber, takes the form [45]

$$
\mathcal{M}(q)=(q)_{\infty}^{-2} \prod_{\substack{\text { Galois } \\ \text { orbits }}}^{\circlearrowright}\left\{\left( \pm q^{1 / 2} X_{\gamma} ; q\right)_{\infty}\left( \pm q^{1 / 2} X_{\iota(\gamma)} ; q\right)_{\infty}\right\}
$$

where the two factors inside each curly bracket commute between themselves by eq. (6.38). 
This model has an $\mathrm{SO}(8)$ flavor symmetry; correspondingly the exchange matrix $B_{i j}$ of the quiver (6.36) has rank 2 , and we may parametrize its quantum torus algebra $\mathbb{T}_{Q}$ in terms of four commuting flavor fugacities $y_{a}$ and two operators $X_{e_{1}}, X_{e_{2}}$ satisfying the $\mathbb{T}_{\vec{A}_{2}}$ quantum torus algebra $X_{e_{1}} X_{e_{2}}=q X_{e_{2}} X_{e_{1}}$ as follows

$$
\begin{aligned}
& X_{c_{1}}=y_{1} X_{e_{1}}, \quad X_{c_{2}}=y_{1} X_{e_{1}}^{-1}, \\
& X_{b_{1}}=y_{2} y_{3} X_{e_{2}}, \quad X_{b_{2}}=y_{2}^{-1} y_{3} X_{e_{2}} \quad X_{a_{1}}=y_{4} y_{3} X_{e_{2}}^{-1}, \quad X_{a_{1}}=y_{4}^{-1} y_{3} X_{e_{2}}^{-1} \text {. }
\end{aligned}
$$

The monodromy $\mathcal{M}(q)$ may then be seen as an element of the $\mathbb{T}_{\vec{A}_{2}}$ algebra which depends on the $y_{a}$ parameters. Although we could be more general, since the flavor fugacity dependence plays a secondary role in our discussion, for simplicity we set $y_{a}=1$. At this special point in flavor fugacity space eq. (6.40) implies

$$
X_{\iota(\gamma)}=X_{\gamma}^{-1},
$$

and hence each curly bracket in eq. (6.39) takes the form

$$
\left\{\left( \pm q^{1 / 2} X_{\gamma} ; q\right)_{\infty}\left( \pm q^{1 / 2} X_{\iota(\gamma)} ; q\right)_{\infty}\right\} \longrightarrow \frac{\theta\left( \pm q^{1 / 2} X_{\gamma} ; q\right)}{(q)_{\infty}}
$$

where $\theta(z ; q)$ is the function defined in eq. (6.16). Hence we may write,

$$
\left.\mathcal{M}(q)\right|_{y_{a}=1}=(q)_{\infty}^{-12 c}\left(L_{4}^{2} L_{3} L_{2} L_{1}\right)^{2}
$$

where $c=14 / 12$ is the $4 \mathrm{~d}$ conformal central charge, and the $L_{i}, \quad(i=1,2,3,4)$ are Kontsevich-Soibelman products in suitably angular sectors containing two (or four) mutually-local stable hypermultiplets. Explicitly,

$$
\begin{array}{ll}
L_{1}=\theta\left(q^{1 / 2} X_{e_{1}} ; q\right) & L_{2}=\theta\left(-q^{1 / 2} X_{e_{2}-e_{1}} ; q\right)^{2} \\
L_{3}=\theta\left(q^{1 / 2} X_{2 e_{2}-e_{1}} ; q\right) & L_{4}=\theta\left(q^{1 / 2} X_{e_{2}} ; q\right) .
\end{array}
$$

Using the functional equation (6.17) for $\theta$, we get

$$
\begin{gathered}
L_{1}\left\{\begin{array}{l}
X_{e_{1}} \\
X_{e_{2}}
\end{array}\right\} L_{1}^{-1}=\left\{\begin{array}{c}
X_{e_{1}} \\
-X_{e_{2}-e_{1}}
\end{array}\right\}, \quad L_{2}\left\{\begin{array}{l}
X_{e_{1}} \\
X_{e_{2}}
\end{array}\right\} L_{2}^{-1}=\left\{\begin{array}{c}
X_{2 e_{2}-e_{1}} \\
X_{3 e_{2}-2 e_{1}}
\end{array}\right\} \\
L_{3}\left\{\begin{array}{l}
X_{e_{1}} \\
X_{e_{2}}
\end{array}\right\} L_{3}^{-1}=\left\{\begin{array}{c}
X_{4 e_{2}-e_{1}} \\
-X_{3 e_{2}-e_{1}}
\end{array}\right\}, \quad L_{4}\left\{\begin{array}{l}
X_{e_{1}} \\
X_{e_{2}}
\end{array}\right\} L_{4}^{-1}=\left\{\begin{array}{c}
X_{e_{1}+e_{2}} \\
X_{e_{2}}
\end{array}\right\},
\end{gathered}
$$

so that the half-monodromy acts as

$$
\left(L_{4}^{2} L_{3} L_{2} L_{1}\right)\left\{\begin{array}{l}
X_{e_{1}} \\
X_{e_{2}}
\end{array}\right\}\left(L_{4}^{2} L_{3} L_{2} L_{1}\right)^{-1}=\left\{\begin{array}{l}
X_{-e_{1}} \\
X_{-e_{2}}
\end{array}\right\}
$$

and the full-monodromy $\mathcal{M}(q)$ as

$$
\mathcal{M}(q)\left\{\begin{array}{l}
X_{e_{1}} \\
X_{e_{2}}
\end{array}\right\} \mathcal{M}(q)^{-1}=\left\{\begin{array}{l}
X_{e_{1}} \\
X_{e_{2}}
\end{array}\right\}
$$


in agreement with eq. (6.12).

We may fix our reference $S$-duality frame so that the operator $X_{a e_{1}+b e_{2}}$ has electric/magnetic charges $(a, b)$. Then eq. (6.45) may be rephrased as the statement that the KS operators $L_{i}$ induce the following $\mathrm{SL}(2, \mathbb{Z}) S$-duality transformations on the electromagnetic charges of the line operators

$$
\begin{aligned}
& L_{1} \rightarrow\left(\begin{array}{cc}
1 & 0 \\
-1 & 1
\end{array}\right), \quad L_{2} \rightarrow\left(\begin{array}{ll}
-1 & 2 \\
-2 & 3
\end{array}\right) \\
& L_{3} \rightarrow\left(\begin{array}{ll}
-1 & 4 \\
-1 & 3
\end{array}\right), \quad L_{4} \rightarrow\left(\begin{array}{ll}
1 & 1 \\
0 & 1
\end{array}\right) .
\end{aligned}
$$

In particular $L_{1}, L_{4}$ suffice to generate the full $\mathrm{SL}(2, \mathbb{Z})$ action on line operators. We conclude that the KS product of BPS factors associated to an angular sector is the same thing as the telescoping operator ${ }^{15}$ implementing the $S$-duality by the corresponding angle.

Equation (6.47) is the same as (6.15) for $N_{f}=4$. It has many solutions. If we assume it to be a function only of the 'electric' fugacity $X_{e_{1}}$, as suggested by the 'oblique confinement' physical picture we wish to check, we have solutions of the general form

$$
\mathcal{M}(q)=f(q) \prod_{a=1}^{K} \theta\left(q^{\ell_{a}} X_{e_{1}}^{m_{a}} ; q\right)^{n_{a}}
$$

with $K$ even and

$$
\sum_{a} n_{a} m_{a}^{2}=0, \quad \sum_{a} m_{a} n_{a}\left(\ell_{a}+\frac{m_{a}-1}{2}\right)=0, \quad \sum_{a} m_{a} n_{a}=0 \quad \bmod 2 .
$$

Two particular solutions to these Diophantine equations correspond to the extreme weak coupling description of the monodromy for the two Lagrangian SCFT with one-dimensional Coulomb branch, i.e. $\mathrm{SU}(2) \mathcal{N}=2^{*}$ and $N_{f}=4$ respectively

$$
\begin{array}{ll}
\text { - } \mathcal{N}=2^{*} & \left(\ell_{a}, m_{a}, n_{a}\right)=\overbrace{(1,2,1),(0,2,1)}^{W \text { boson }}, \overbrace{(1 / 2,2,-1)(1 / 2,2,-1)}^{\text {adj. quark }} \\
\text { - } N_{f}=4 \quad\left(\ell_{a}, m_{a}, n_{a}\right)=\overbrace{(1,2,1),(0,2,1)}^{W \text { boson }}, \overbrace{(1 / 2,2,-1)(1 / 2,2,-1) \cdots(1 / 2,1,-1)}^{(8 \text { times }) \text { quarks }}
\end{array}
$$

These are (essentially) the unique solutions if we require the singularities to be of the respective 'right' form (i.e. singularities allowed only if they may arise from quarks becoming massless).

\footnotetext{
${ }^{15}$ These operators correspond to the mathematicians' telescopic (endo)functors in the corresponding derived category [46]. For a review in the present physical context, see [47]. In the categoric language, the BPS states of $\mathrm{SU}(2)$ with $N_{f}$ quarks are the stable objects in the derived category $\mathrm{D}^{b}\left(\right.$ coh $\mathbb{P}_{N_{f}}$ ) of coherent sheaves on the orbifold of $\mathbb{P}^{1}$ with $N_{f}$ double points. The quantum monodromy $\mathcal{M}(q)$ is the autoequivalence $\left(\tau^{-1}[1]\right)^{2}$ of $\mathrm{D}^{b}\left(\operatorname{coh} \mathbb{P}_{N_{f}}\right)[6]$. The physical equations (6.12) are just Serre duality on $\mathbb{P}_{N_{f}}$. In particular, the monodromy is an autoequivalence of the Abelian category of objects with $m=0, e>0$, (the category of finite-length coherent sheaves) i.e. $\mathcal{M}(q) X_{e} \mathcal{M}(q)^{-1}=X_{e}$. If the orbifold $\mathbb{P}_{N_{f}}$ has zero Euler characteristic, i.e. for $N_{f}=4$, we have also $\mathcal{M}(q) X_{m} \mathcal{M}(q)^{-1}=X_{m}$.
} 
General KS theory implies, in particular, that the monodromies written with respect to two different $S$-duality frames are equal modulo conjugacy, more precisely that they differ by the adjoint action of an operator which is a KS product of BPS factors. Then consider the monodromy written with respect to the frame in which the weakly coupled $W$ boson has charge $2\left(p e_{1}+q e_{2}\right)$ with $p, q$ coprime. If the monodromy in the original $S$-frame was given by equation (6.49), the monodromy in the new frame should be simply

$$
\mathcal{M}(q)^{\text {new }}=f(q) \prod_{a=1}^{K} \theta\left(q^{\ell_{a}} X_{p e_{1}+q e_{2}}^{m_{a}} ; q\right)^{n_{a}}
$$

i.e. the same function where we replaced $X_{e_{1}}$ by $X_{p e_{1}+q e_{2}}$. Consistency of the physical picture of monodromy proposed in section 6.2 with the Kontsevich-Soibelman formula then requires that there exists an operator $W \in \mathbb{T}$ such that

$$
\mathcal{M}(q)^{\text {new }}=W \mathcal{M}(q)^{\text {old }} W^{-1},
$$

and moreover that $W$ can be chosen to be equal to the product of BPS factors with phases in the range $\arg Z_{e_{1}}<\theta<\arg Z_{p e_{1}+q e_{2}}$. The existence of a $W$ with the prescribed properties for all pair of coprime integers $(p, q)$ is a significant check on the physical scenario.

The existence of $W$ follows from the previous monodromy computation in the strong coupling chamber. For each $(p, q)$ coprime there is an $A \in \mathrm{SL}(2, \mathbb{Z})$ which transforms $e_{1}$ into $p e_{1}+q e_{2}$. Since $L_{1}, L_{4}$ generates $\operatorname{SL}(2, \mathbb{Z})$, there is a word $W$ in $L_{1}, L_{2}$ equal to $A$. Then

$$
W X_{e_{1}} W^{-1}=X_{a e_{1}+b e_{2}}
$$

and eq. (6.54) is satisfied. By construction, $W$ is a product of BPS factors $\left( \pm q^{1 / 2} X_{\gamma}, q\right)_{\infty}$. So the physical picture passes this check of consistency with KS theory.

\subsection{A non-Lagrangian example}

The above results for $\mathrm{SU}(2)$ with $N_{f}=4$ generalize to all $\mathcal{N}=2$ SCFTs with a finite chamber such that all operators have integral $\mathrm{U}(1)_{R}$ charge [6], even if they do not have a Lagrangian description. When the Coulomb branch has dimension 1 , one gets a $\mathrm{SL}(2, \mathbb{Z})$ action on the line operators $X_{\gamma}$ implemented by the KS products which is similar to the one we described for $\mathrm{SU}(2)$ with $N_{f}=4$. If the model has no Lagrangian formulation, the physical implications of this $\mathrm{SL}(2, \mathbb{Z})$ auto-equivalence is less obvious since we have no weak coupling intuition. We illustrate these facts in the simplest non-Lagrangian model of this class.

$\mathrm{SU}(2)$ with $N_{f}=4$ is a special instance of a sequence of $\mathcal{N}=2$ models with onedimensional Coulomb branch and large flavor symmetry. They are described by the class of the $Q(r, s)$ quivers in figure 2 where $r, s$ are non-negative integers not both zero. These models have a non-Abelian flavor symmetry of rank $r+s$ described by the Dynkin graph in the lower part of the figure. Since the flavor symmetry group of a meaningful QFT must be compact, only pairs $(r, s)$ such that the lower graph is a Dynkin graph of finitetype correspond to a $\mathcal{N}=2$ model. In particular, $Q\left(2, N_{f}\right)$ is the quiver of $\mathrm{SU}(2)$ with $N_{f}$ quarks, and $Q(3,3), Q(3,4)$ and $Q(3,5)$ are the quiver of the Minahan-Nemeschansky theories with flavor symmetry $E_{r+s}$. 

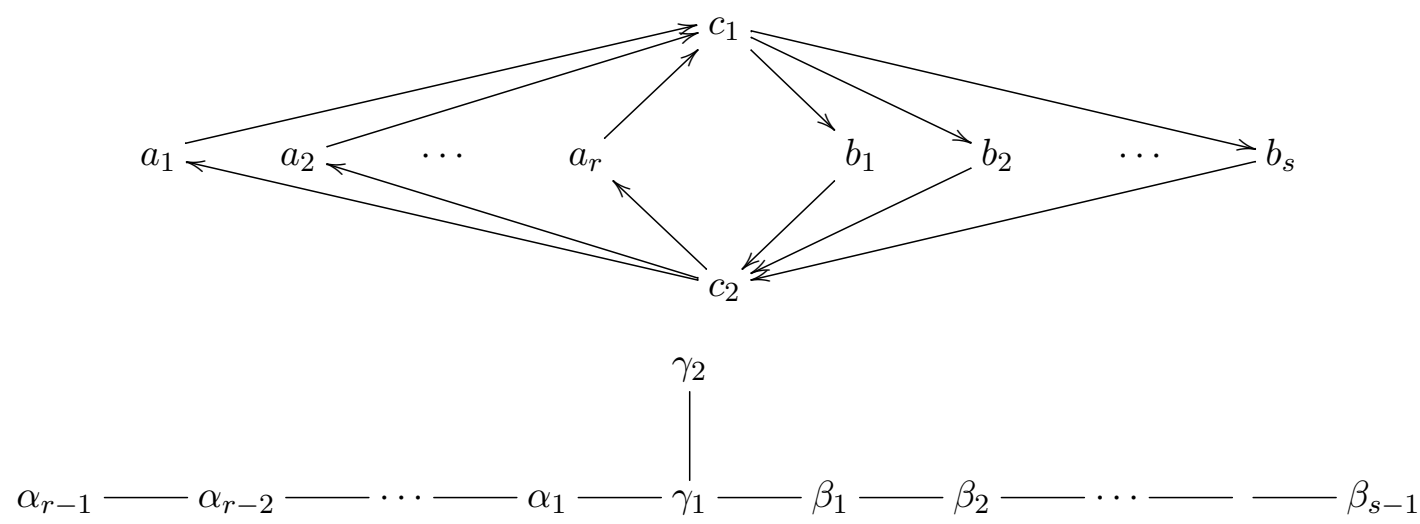

Figure 2. The $Q(r, s) \equiv Q(s, r)$ quiver $(r+s)>0$. The corresponding $\mathcal{N}=2$ theory has onedimensional Coulomb branch and rank $(r+s)$ flavor symmetry group $G_{F}$ with the Dynkin graph in the lower graph.

The SCFTs described by a $Q(r, s)$ quiver — which are known as $H_{1}, H_{2}, D_{4}, E_{6}, E_{7}, E_{8}$ - as well as many others like the Argyres-Douglas theories of type $D$, have in common some remarkable properties that we shall use in section 7 as a guiding principle to compute in an efficient way their monodromy traces. From the viewpoint of the $2 \mathrm{~d} / 4 \mathrm{~d}$ correspondence of [8] the property may be stated as follows: these SCFT have a flavor group $G_{F}$ with a 4 d level $k$ and a conformal central charge $c$ to which there correspond the 2d CFT quantities $c_{2 d}=-12 c$ and $k_{2 d}=-k / 2$. These models have the special property that the 2d Sugawara energy-momentum tensor of the flavor symmetry $G_{F}$ saturates the Virasoro central charge, i.e. we have the equality

$$
c_{2 d}=\frac{k_{2 d} \cdot \operatorname{dim} G_{F}}{h_{G_{F}}+k_{2 d}} .
$$

The same SCFTs were shown in [44] to have a finite BPS chamber which is $c$-saturating, i.e. such that the conformal central charge $c$ computed from the BPS spectrum pretending it consists of free particles is, for that particular chamber, equal to its actual value. The $c$-saturating chambers lead to very convenient expressions for the monodromy which greatly simplify the task of computing their traces. A first example of $c$-saturating is the 12 hyper chamber of SU(2) with $N_{f}=4$ : in eq. (6.43) the monodromy, as written in the $c$-saturating chamber, is equal to $(q)_{\infty}^{-12 c}$ times a product of theta functions. This formula is true (for an appropriate product of theta functions) for all rank 1 SCFT models in the above list (again switching off flavor fugacities). In particular, experience suggests that in all SCFTs for which (6.56) holds, for all $N>0$ such that $\operatorname{Tr} \mathcal{M}(q)^{N}$ makes sense, we have

$$
\operatorname{Tr} \mathcal{M}(q)^{N}=\frac{\boldsymbol{\Theta}_{N}\left(y_{a} ; q\right)}{(q)_{\infty}^{12 N c}}
$$

where $c$ is the $4 \mathrm{~d}$ SCFT central charge and $\boldsymbol{\Theta}_{N}\left(y_{a} ; q\right)$ is the theta-function of some lattice. In section 7 we shall find many instance of this phenomenon so helpful in computing the traces. 
For brevity we discuss only the first Minahan-Nemeschansky model with $G_{F}=E_{6}$. Its quiver $Q(3,3)$ also has a $\mathbb{Z}_{2}$ Galois automorphism $\iota$

$$
a_{i} \stackrel{\iota}{\longleftrightarrow} b_{i} \quad c_{1} \stackrel{\iota}{\longleftrightarrow} c_{2},
$$

which is a symmetry of the BPS spectrum in the finite $c$-saturating 24 hypers chamber. Since the Coulomb branch is one-dimensional, we may parametrize the quantum torus algebra $\mathbb{T}_{Q(3,3)}$ in terms of the algebra $\mathbb{T}_{\vec{A}_{2}}, X_{e_{1}} X_{e_{2}}=q X_{e_{2}} X_{e_{1}}$ and six flavor fugacities $y_{a}$. Setting the $E_{6}$ fugacities to 1 , we again get $X_{\iota(\gamma)}=X_{\gamma}^{-1}$ and, by the same argument we used for $\mathrm{SU}(2)$ with $N_{f}=4$, the monodromy reads

$$
\mathcal{M}(q)=(q)_{\infty}^{-12 c}\left(L_{6} L_{5} L_{4} L_{3} L_{2} L_{1}\right)^{2}
$$

where $c=13 / 6$ is the model's conformal central charge and

$$
\begin{array}{lll}
L_{1}=\theta\left(q^{1 / 2} X_{e_{1}} ; q\right) & L_{2}=\theta\left(-q^{1 / 2} X_{e_{2}-e_{1}} ; q\right)^{3} & L_{3}=\theta\left(q^{1 / 2} X_{3 e_{2}-2 e_{1}} ; q\right) \\
L_{4}=\theta\left(q^{1 / 2} X_{2 e_{2}-e_{1}} ; q\right)^{3} & L_{5}=\theta\left(-q^{1 / 2} X_{3 e_{2}-e_{1}} ; q\right) & L_{6}=\theta\left(q^{1 / 2} X_{e_{2}} ; q\right)^{3} .
\end{array}
$$

Using (6.17), we get

$$
\begin{aligned}
& L_{1}\left\{\begin{array}{l}
X_{e_{1}} \\
X_{e_{2}}
\end{array}\right\} L_{1}^{-1}=\left\{\begin{array}{c}
X_{e_{1}} \\
-X_{e_{2}-e_{1}}
\end{array}\right\}, \quad L_{2}\left\{\begin{array}{l}
X_{e_{1}} \\
X_{e_{2}}
\end{array}\right\} L_{2}^{-1}=\left\{\begin{array}{c}
-X_{3 e_{2}-2 e_{1}} \\
-X_{4 e_{2}-3 e_{1}}
\end{array}\right\} \\
& L_{3}\left\{\begin{array}{l}
X_{e_{1}} \\
X_{e_{2}}
\end{array}\right\} L_{3}^{-1}=\left\{\begin{array}{l}
-X_{9 e_{2}-5 e_{1}} \\
-X_{7 e_{2}-4 e_{1}}
\end{array}\right\}, \quad L_{4}\left\{\begin{array}{l}
X_{e_{1}} \\
X_{e_{2}}
\end{array}\right\} L_{4}^{-1}=\left\{\begin{array}{c}
X_{12 e_{2}-5 e_{1}} \\
-X_{7 e_{2}-3 e_{1}}
\end{array}\right\} \text {, } \\
& L_{5}\left\{\begin{array}{l}
X_{e_{1}} \\
X_{e_{2}}
\end{array}\right\} L_{5}^{-1}=\left\{\begin{array}{c}
-X_{9 e_{2}-2 e_{1}} \\
-X_{4 e_{2}-e_{1}}
\end{array}\right\}, \quad L_{6}\left\{\begin{array}{l}
X_{e_{1}} \\
X_{e_{2}}
\end{array}\right\} L_{6}^{-1}=\left\{\begin{array}{c}
-X_{e_{1}+3 e_{2}} \\
X_{e_{2}}
\end{array}\right\}
\end{aligned}
$$

so that the adjoint action of each $L_{i}$, and hence of their products, acts (a part for the signs from quadratic refinement) is just a $\mathrm{SL}(2, \mathbb{Z})$ transformation

$$
L_{i} X_{\gamma} L_{i}^{-1}=X_{A_{i}(\gamma)} \quad A_{i} \in \mathrm{SL}(2, \mathbb{Z}) .
$$

However now the half-monodromy acts as the identity

$$
\left(L_{6} L_{5} \cdots L_{1}\right)\left\{\begin{array}{c}
X_{e_{1}} \\
X_{e_{2}}
\end{array}\right\}\left(L_{6} L_{5} \cdots L_{1}\right)^{-1}=\left\{\begin{array}{c}
X_{e_{1}} \\
X_{e_{2}}
\end{array}\right\} .
$$

The situation for $E_{7}$ and $E_{8}$ Minahan-Nemeschansky is similar, but the monodromy has a less compact expression since these models have no Galois symmetry. The analysis of their monodromy in the classical limit $q \rightarrow 1$ (but general flavor fugacities) may be found in [48].

\subsection{Generalities on monodromy traces}

Having discussed the Lagragian cases and some extensions of them, in this section we turn to some general properties of the monodromy traces which follow from their formal 
structure. Appendix $\mathrm{C}$ contains a survey of the main techniques to compute monodromy traces. The interested reader can find more details and explicit expressions for all quantities related to the traces that we will need.

The methods of [6] work for models having a finite BPS chamber i.e. a chamber whose BPS spectrum consists of finitely many hypermultiplets; then the KS product $M(q)^{N}$ contains finitely many factors $\left(q^{1 / 2} X_{\gamma} ; q\right)_{\infty}$. One expands each such factor in the basis $\left\{X_{\gamma}\right\}_{\gamma \in \Gamma}$ of the quantum torus algebra $\mathbb{T}$ and then multiplies them with the rule (6.2) to get a (formal) expression of the form

$$
\mathcal{M}(q)^{N}=\sum_{\gamma \in \Gamma} \mu(N ; q)_{\gamma} X_{\gamma}
$$

for certain coefficient functions $\left\{\mu(N ; q)_{\gamma}\right\}_{\gamma \in \Gamma}$ given by multiple $q$-hypergeometric sums. The trace on the quantum torus algebra $\mathbb{T}$ is defined by the rule ${ }^{16}$

$$
\operatorname{Tr} X_{\gamma}= \begin{cases}y_{\gamma} & \gamma \text { is a flavor charge } \\ 0 & \text { otherwise }\end{cases}
$$

A charge $\gamma$ is a flavor charge iff it belongs to the radical of the Dirac form, i.e. $\langle\gamma, \nu\rangle=0$ for all $\nu \in \Gamma$. The flavor charges form a sublattice $\Gamma_{f} \subset \Gamma$ of the charge lattice. From (6.64), (6.65) the monodromy traces, as functions of $q$ and the flavor fugacities $y_{a}$, are then

$$
\operatorname{Tr} \mathcal{M}(q)^{N}=\sum_{\phi \in \Gamma_{f}} \mu(N ; q)_{\phi} y_{\phi}
$$

The computation of $\operatorname{Tr} \mathcal{M}(q)^{N}$ is thus reduced to the evaluation of $q$-hypergeometric sums $\left\{\mu(N ; q)_{\phi}\right\}_{\phi \in \Gamma_{f}}$ which have the general form [6]

$$
\sum_{n_{1}, \ldots, n_{s} \geq 0} \frac{q^{Q\left(n_{i}\right) / 2} \prod_{i} z_{i}^{n_{i}}}{\prod_{i}(q)_{n_{i}}} \prod_{a} \delta_{b_{a i} n_{i}, t_{a}}
$$

where $Q\left(n_{i}\right) \equiv n_{i} A_{i j} n_{j}$ is an integral quadratic form and the Kronecker deltas enforce the proper restriction on the summation range; see appendix $\mathrm{C}$ for full details, including explicit expressions for all elements appearing in (6.67). $q$-sums of this form are familiar from the thermodynamical Bethe ansatz (TBA) and related $Y$-systems, see e.g. [49]. Ideas and techniques developed in those contexts may be applied also to monodromy traces. Depending on $N$ and the particular model at hand, the sum (6.67) may or may not be absolutely convergent; if not an appropriate prescription is required to define $\operatorname{Tr} \mathcal{M}(q)^{N}$.

\footnotetext{
${ }^{16}$ This is slightly different from the notion of 'canonical trace' used in [6] where the trace included a projection also to flavor neutral sector. The relation between the two notions of trace is as follows:

$$
\left.\operatorname{Tr} \mathcal{M}(q)^{N}\right|_{\substack{\text { sNV } \\ \text { sense [6] }}}=\left.\oint \operatorname{Tr} \mathcal{M}(q)^{N}\right|_{\substack{\text { present } \\ \text { sense }}} \prod_{a} \frac{d y_{a}}{2 \pi i y_{a}} .
$$
}


Moyal functions. Let $\left\{e_{s}\right\}$ be a set of generators of the charge lattice $\Gamma$, and set $B_{s, t}=$ $\left\langle e_{s}, e_{t}\right\rangle$. The quantum torus algebra $\mathbb{T}$, which has generators $\left\{X_{e_{s}}\right\}$ and commutation relations $X_{e_{s}} X_{e_{t}}=q^{B_{s t}} X_{e_{t}} X_{e_{s}}$, is isomorphic to the algebra of holomorphic functions on

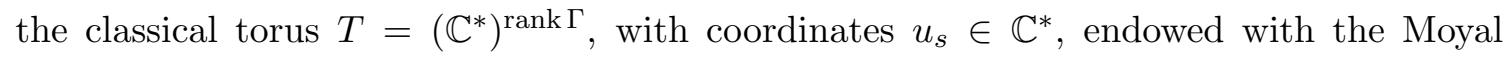
product $*$ defined by the 2 -vector $B_{t s}$, i.e.

$$
f * g\left(u_{s}\right)=\left.\exp \left(\pi i \tau B_{s t} x_{s} y_{t} \partial_{x_{s}} \partial_{y_{t}}\right) f\left(u_{s}+x_{s}\right) g\left(u_{s}+y_{s}\right)\right|_{x=y=0} \quad \text { where } q=e^{2 \pi i \tau} \text {. }
$$

To the charge $\gamma=\sum_{s} n_{s} e_{s} \in \Gamma$ we associate the function $u_{\gamma}=\prod_{s} u_{s}^{n_{s}}$ on $T$. If $\gamma$ is a flavor charge, the corresponding function $u_{\gamma}$ is called a flavor fugacity. In the basis $\left\{X_{\gamma}\right\}$ of $\mathbb{T}$ the isomorphism between $\mathbb{T}$ and the Moyal functions is simply

$$
X_{\gamma} \leftrightarrow u_{\gamma} .
$$

Comparing with (6.64), we see that the Moyal function $\mu\left(u_{s} ; q\right)^{(N)}$ on $T$ which corresponds to the operator $\mathcal{M}(q) \in \mathbb{T}$ is

$$
\mu\left(u_{s} ; q\right)^{(N)}=\sum_{\gamma} \mu(N ; q)_{\gamma} u_{\gamma}
$$

Then

$$
\operatorname{Tr} \mathcal{M}(q)^{N}=\oint \frac{d v_{s}}{2 \pi i v_{s}} \mu\left(u_{s} ; q\right)^{(N)}
$$

where the integral is on the unit circle $\left|u_{s}\right|=1$ at fixed values of the flavor fugacities $y_{a}$. The Moyal formalism has two advantages with respect to the quantum torus algebra: first the quantum cluster mutations [6] may be rephrased as functional equations for the Moyal functions which are often easier to solve. Second, it is usually simpler to find a prescription to make convergent an integral like (6.71) than a $q$-series.

In appendix $\mathrm{C}$ it is shown that the integral (6.71) may be recast in the form $\left(q=e^{2 \pi i \tau}\right)$

$$
\int_{\mathcal{C}} \exp \left(-\frac{S\left(t_{i}, y_{a} ; \tau\right)^{(N)}}{2 \pi i \tau}\right) \prod_{i} d t_{i},
$$

where $S\left(t_{i}, y_{a} ; \tau\right)^{(N)}$ is a function which is regular as $\tau \rightarrow 0$ (see appendix C for its explicit form). The integral (6.71) belongs to the well known class of "oscillatory integrals", which may be interpreted as brane amplitudes $\int_{\mathcal{C}} d X_{i} \exp \left(-W\left(X_{i}\right) / \zeta\right)$ for $2 \mathrm{~d}(2,2)$ SCFTs [50]. For such an integral, giving the appropriate convergence prescription amounts to specifying the correct integration contour $\mathcal{C}$. We stress that, while the contour $\mathcal{C}$ (and hence the proper definition of $\left.\operatorname{Tr} \mathcal{M}(q)^{N}\right)$ may be rather subtle, the function $S\left(t_{i}, y_{a} ; \tau\right)^{(N)}$ contains information on the quantum traces $\operatorname{Tr} \mathcal{M}(q)^{N}$ which is both very valuable and totally non-ambiguous.

The effective central charge $\boldsymbol{c}_{\text {eff }}$. In particular the function $S\left(t_{i}, y_{a} ; \tau\right)^{(N)}$ contains the "2d CFT data", $c_{\text {eff }}$ and $\left\{h_{i}\right\}$, associated with the monodromy trace $\operatorname{Tr} \mathcal{M}(q)^{N}$. These data are defined as follows: view $\operatorname{Tr} \mathcal{M}(q)^{N}$ as a power series in $q$

$$
\operatorname{Tr} \mathcal{M}(q)^{N}=\sum_{n \geq 0} a_{n}(y) q^{n}
$$


whose coefficients $a_{n}(y)$ are Laurent polynomials in the flavor fugacities $y_{a}$ with integral coefficients. The effective central charge $c_{\text {eff }}$ of the power series $\sum_{n \geq 0} a_{n} q^{n}$ is defined as

$$
c_{\mathrm{eff}}=\frac{3}{2 \pi^{2}} \lim _{n \rightarrow \infty} \frac{\left(\log a_{n}\right)^{2}}{n},
$$

or equivalently (setting $q=e^{2 \pi i \tau}$ ) by its $\tau \rightarrow 0$ asymptotic behavior

$$
\sum_{n \geq 0} a_{n} q^{n} \simeq \exp \left(\frac{2 \pi i}{\tau} \frac{c_{\mathrm{eff}}}{24}+O(1)\right) \quad \text { as } \tau \rightarrow 0 .
$$

The name 'effective central charge' stems from the fact that, when the $q$-series (6.73) is a conformal block of a (not necessarily unitary) $2 \mathrm{~d}$ CFT, one has

$$
c_{\text {eff }} \equiv c-24 \min _{i}\left(h_{i}\right)
$$

with $h_{i}, c$ the conformal weights and Virasoro central charge of the CFT. In particular, for unitary CFTs, $c_{\text {eff }} \equiv c$. If there are flavor symmetries, from eq. (6.74) we get a function of the flavor fugacities, $c_{\mathrm{eff}}\left(y_{a}\right)$; in this case, we define the effective central charge $c_{\text {eff }}$ as the value of this function at a (suitable) critical point, $\partial_{y_{a}} c_{\mathrm{eff}}(y)=0$.

To compute $c_{\text {eff }}$, it suffices to evaluate the integral (6.72) in the limit $\tau \rightarrow 0$. This can be done by standard saddle point techniques: $c_{\text {eff }}$ for $\operatorname{Tr} \mathcal{M}(q)^{N}$ is essentially the value of the function $S\left(t_{i}, y_{a} ; \tau\right)^{(N)}$ at its dominating critical point. The saddle point equations may be solved explicitly by adapting standard TBA methods [49] to our situation. The details may be found in appendix C. Here we quote the final result: $c_{\text {eff }}$ for $\operatorname{Tr} \mathcal{M}(q)^{N}$ (as computed from a chamber with $h$ hypers) is given by

$$
c_{\mathrm{eff}}=2 r N+2|N| \sum_{i=1}^{h} \frac{6}{\pi^{2}} L\left(z_{i}\right)
$$

where $L(z)$ is the Roger dilogarithm

$$
L(z)=\operatorname{Li}_{2}(z)+\frac{1}{2} \log z \log (1-z)
$$

and the $z_{i}$ are the solutions to the Nahm-like equations

$$
z_{i}^{2}= \begin{cases}\left(1-z_{j}\right)^{C_{i j}}, & N>0 \\ \left(1-z_{j}\right)^{2 \delta_{i j}-C_{i j}} & N<0\end{cases}
$$

where $C_{i j}$ is the integral symmetric $h \times h$ matrix specified in appendix $\mathrm{C}$.

Below we will need to recall that $c^{4 d}, a^{4 d}$ for $\mathrm{AD}\left(A, A^{\prime}\right)$ theory:

$$
\begin{aligned}
c^{4 d} & =\frac{\operatorname{dim} A \operatorname{dim} A^{\prime}-r_{A} r_{A^{\prime}}}{12\left(h_{A}+h_{A^{\prime}}\right)} \\
a^{4 d} & =\frac{4 \operatorname{dim} A \operatorname{dim} A^{\prime}-5 r_{A} r_{A^{\prime}}}{48\left(h_{A}+h_{A^{\prime}}\right)}
\end{aligned}
$$


Example 1: $\left(\boldsymbol{G}, \boldsymbol{A}_{\mathbf{1}}\right)$ models with $\boldsymbol{N}>\mathbf{0}$. In this case the equations (6.79) take the form

$$
z_{i}^{2}=\left(1-z_{j}\right)^{C_{i j}}
$$

where $C_{i j}$ the Cartan matrix of the simply-laced Lie algebra $G$. Writing $z_{i}=w_{j}^{-C_{i j}}$, the equations take the form

$$
1+w_{j}^{2 \delta_{i j}-C_{i j}}=w_{i}^{2} .
$$

The equations (6.80) have a unique solution with $0<z_{i}<1$ which corresponds to the vacuum character of the $2 \mathrm{~d}$ coset $\mathrm{CFT} G_{(2)} / \mathrm{U}(1)^{r_{G}}[49,51]$. Then $6 \sum_{i} L\left(z_{i}\right) / \pi^{2}$ is just the central charge of the $G_{(2)} / \mathrm{U}(1)^{r_{G}}$ coset CFT, and

$$
c_{\mathrm{eff}}=2 N\left(r+\frac{r_{G} h_{G}}{h_{G}+2}\right),
$$

where $r$ is the rank of the Coulomb branch of the AD theory, and $r_{G}, h_{G}$ are the rank and the Coxeter number of the Lie algebra $G$ (related to its dimension by the Coxeter formula $\left.\operatorname{dim} G=r_{G}\left(h_{G}+1\right)\right) .2 r$ is equal to $r_{G}$ minus the multiplicity of $h_{G} / 2$ as an exponent of $G$. For $\left(A_{1}, G\right)$ Argyres-Douglas theories the $12 N c^{4 d}$ is expected to be given by the above formula for $c_{\text {eff }}$ and this is in agreement with the expected answer $\left(A_{1}, A_{r_{G}}\right)$.

Example 2: $\left(\boldsymbol{G}, \boldsymbol{A}_{\mathbf{1}}\right)$ models with $\boldsymbol{N}<\mathbf{0}$. In this case the equations (6.79) take the form

$$
z_{i}^{2}=\left(1-z_{j}\right)^{2 \delta_{i j}-C_{i j}}
$$

Setting $z_{i}=-w_{j}^{2 \delta_{i j}-C_{i j}}$ we get back the Nahm equations (6.81). For $N<0$ we are interested in a different solution of these equation. We may find it by the same Lie theoretic methods as for $N>0$ [49]. For instance, for the $\left(A_{1}, A_{2 \ell}\right)$ model with $N<0$ one finds (appendix C)

$$
c_{\text {eff }}=\frac{2|N| \ell}{2 \ell+3} .
$$

Note that this agree with the expected answer $-48 N\left(c^{4 d}-a^{4 d}\right)$ for the AD theory when $G=A_{2 l}$.

The effective central charge of the $(p, q)$ Virasoro minimal model is

$$
c_{\mathrm{eff}}(p, q)=c-24 \min _{r, s} h_{r, s}=1-\frac{6}{p q} .
$$

Taking $p=2$ and $q=2 \ell+3$, we get the effective central charge (6.84) for $N=-1$. Note that this agrees with the general prediction that $h_{\min }=(-1 / 2)\left(5 c^{4 d}-4 a^{4 d}\right)$.

Example 3: $\left(G, G^{\prime}\right)$ models with $N>0$. Again we reduce the saddle point conditions to systems of algebraic equations already studied in a related context by Nahm [49]. Using his results, one obtains the following formula

$$
c^{2 d}=c_{\mathrm{eff}}=2 N r+N \frac{r_{G} r_{G^{\prime}} h_{G} h_{G^{\prime}}}{h_{G}+h_{G^{\prime}}}=12 N c^{4 d},
$$


which has been checked explicitly for $\left(G, A_{2}\right)$. Using this and the $r$-charges for the Coulomb branch of the $\left(G, G^{\prime}\right)$ models which are known, one can also deduce $a^{4 d}$ for all $\left(G, G^{\prime}\right)$ AD theories from the relation $[22]$

$$
4\left(2 a^{4 d}-c^{2 d}\right)=\sum_{i}\left(2 r_{i}-1\right),
$$

where the sum is over all the Coulomb branch operators with dimension given by $r_{i}$.

\section{Monodromies and 2d chiral algebras of Argyres-Douglas theories}

\subsection{Insertion of line operators and characters of chiral algebras}

The Argyres-Douglas (AD) theories are isolated fixed points of $4 \mathrm{~d} \mathcal{N}=2$ supersymmetric field theories with finite number of BPS states in all chambers [52]. Therefore to write down $q$-series expressing $\operatorname{Tr} \mathcal{M}(q)^{N}$ is straightforward. (But, for large $|N|$, the resulting $q$-series are not absolutely convergent, and suitable prescriptions are required to make sense out of them). On the other hand, the computation of the supersymmetric indices $\mathcal{I}_{N}(q)$ of $\mathrm{AD}$ theories is difficult even in principle, because most of them do not have a Lagrangian description. In view of the proposed correspondence (6.4), the "easy" computation of the monodromy traces may be used as a prediction of superconformal indices in the limit $t=q p^{N+1}$ and $p \rightarrow \exp (2 \pi i)$.

More generally, we may consider monodromy traces with line operator insertions

$$
\left\langle X_{\gamma}\right\rangle_{N}=\operatorname{Tr}\left[\mathcal{M}(q)^{N} X_{\gamma}\right] \quad \gamma \in \Gamma .
$$

We stress that the $\left\langle X_{\gamma}\right\rangle_{N}$ are not absolute invariants, since $\mathcal{M}(q)$ is unique only up to conjugacy. $\mathcal{M}(q)$ depends on the choice of a reference ray in the $Z$-plane, which conventionally we take to be the positive real axis. $\left\langle X_{\gamma}\right\rangle_{N}$ is invariant only under the deformations of the parameters such that no central charge $Z_{\gamma}$ of a BPS particle crosses the real axis. In short, in the standard quiver formulation [39] the $\left\langle X_{\gamma}\right\rangle_{N}$ depend on the choice of a quiver in the mutation class, but not on the point in parameter space, as long as it is covered by the given quiver.

Fixing the quiver $Q$, the $\left\langle X_{\gamma}\right\rangle_{N}$ may be read directly from the expansion of the operator $\mathcal{M}(q)^{N}$ in the standard basis of the quantum torus algebra $\mathbb{T}$ (see eq. (6.64))

$$
\mathcal{M}(q)^{N}=\sum_{\gamma \in \Gamma}\left\langle X_{\gamma}\right\rangle_{N} X_{-\gamma}
$$

The $\left\langle X_{\gamma}\right\rangle_{N}$ may be used to compute traces of powers of $\mathcal{M}(q)^{N}$. For instance

$$
\operatorname{Tr} \mathcal{M}(q)^{2 N}=\sum_{\gamma \in \Gamma}\left\langle X_{\gamma}\right\rangle_{N}\left\langle X_{-\gamma}\right\rangle_{N}
$$

In this section we choose the quiver to have the Dynkin form with the linear orientation.

\section{$7.2\left(A_{1}, A_{2}\right)$ AD theory}

Let us start the discussion with the $\mathrm{AD}$ theory of the $\left(A_{1}, A_{2}\right)$ singularity. The BPS quiver of this theory is shown in figure 3 . 


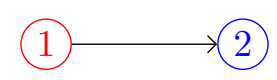

Figure 3. BPS quiver.

Trace of $\mathcal{M}(\boldsymbol{q})^{-1}$. The trace of the inverse monodromy for the $\left(A_{1}, A_{2}\right)$ theory was first computed in [6]. The result is (see appendix D.2)

$$
\operatorname{Tr} \mathcal{M}(q)^{-1}=H(q)
$$

where $H(q)$ is the Rogers-Ramanujan function,

$$
H(q)=\sum_{l=0}^{\infty} \frac{q^{l^{2}+l}}{(q)_{l}}=\prod_{n=1}^{\infty} \frac{1}{\left(1-q^{5 n-2}\right)\left(1-q^{5 n-3}\right)} .
$$

It is known that $q^{11 / 60} H(q)$ is the vacuum character $\chi_{1,1}$ of the $(2,5)$ Virasoro minimal model. The central charge of the $(2,5)$ minimal model is $-22 / 5$, with matches our prediction in table 6 of appendix B (see also equation (C.49)). The central charge of the $2 \mathrm{~d}$ chiral algebra is -12 times the central charge of the $4 \mathrm{~d}$ theory as noted in $[8,9,53]$.

Trace of $\mathcal{M}(\boldsymbol{q})$. The monodromy trace $\operatorname{Tr} \mathcal{M}(q)$ is equal to [6]

$$
\operatorname{Tr} \mathcal{M}(q)=\frac{G(q)}{(q)_{\infty}^{4}}
$$

where $G(q)$ is the other Rogers-Ramanujan function,

$$
G(q)=\prod_{k=1}^{\infty} \frac{1}{\left(1-q^{5 n-1}\right)\left(1-q^{5 n-4}\right)} .
$$

The effective central charge of $\operatorname{Tr} \mathcal{M}(q)$ is $22 / 5$ as shown in table 6 (cfr. equation (C.45)). Indeed, $q^{-1 / 60}(q)_{\infty}^{4} \operatorname{Tr} \mathcal{M}(q)$ is the character $\chi_{1,3}$ of the $(2,5)$ minimal model which has an effective central charge

$$
c_{\mathrm{eff}}=1-\frac{6}{2 \cdot 5} \equiv \frac{22}{5}-4 .
$$

We have provided evidence in the Lagrangian cases that at least in the extreme weak limit we have a chiral algebra, whose character is computed by the trace of the monodromy. We now can test this for the $\operatorname{Tr} \mathcal{M}(q)$. Which chiral algebra has the character $G(q) / \eta(q)^{4}$ ? Note that $G(q)$ is a character of a representation of the $(2,5)$ model but it is not the character of the corresponding algebra (i.e. of the vacuum module). So our general conjecture anticipates that there should be nevertheless another chiral algebra whose vacuum character gives $G(q) / \eta(q)^{4}$. The $1 / \eta^{4}$ is simply the chiral algebra associate to 4 free boson (with zero modes deleted). So the question is whether we can view $G(q)$ as the character of a chiral algebra. 
The chiral algebra $\mathcal{A}_{1}$ associated to $\operatorname{Tr} \mathcal{M}(\boldsymbol{q})$. As shown in [54],G(q) is the vacuum character of the subalgebra of $\mathrm{SU}(2)$ at level $k=1$ generated by $J^{+}(z)=J^{1}(z)+i J^{2}(z)$. For level 1 this algebra is simple and has only one relation given by

$$
J^{+}(z) J^{+}(z)=0 .
$$

Let us see why the characters of this algebra is given by $G(q)$. To see this we rewrite $G(q)$ as

$$
G(q)=\sum_{n=0}^{\infty} \frac{q^{n^{2}}}{\prod_{i=1}^{n}\left(1-q^{i}\right)}=1+\frac{q}{1-q}+\frac{q^{4}}{(1-q)\left(1-q^{2}\right)}+\cdots
$$

Clearly $J^{+}(z)$ and its derivatives gives the module $q /(1-q)$. There is no $q^{2}$ and $q^{3}$ term because of the relation $\partial\left(J^{+}(z) J^{+}(z)\right)=0$ and $J^{+}(z) \partial J^{+}(z)=\partial\left(J^{+}(z) J^{+}(z)\right)=0$. The module $q^{4} /\left((1-q)\left(1-q^{2}\right)\right)$ comes from $\partial J^{+}(z) \partial J^{+}(z)$ and its derivatives, the denominator is $(1-q)\left(1-q^{2}\right)$ instead of $(1-q)^{2}$ is due to the symmetry between two $\partial J^{+}(z)$ 's. The algebra behind positive monodromy $\operatorname{Tr} \mathcal{M}(q)$ is just the aforementioned $J^{+}$subalgebra of $\mathrm{SU}(2)_{1}$ together with four free scalars. One interesting fact is that $\operatorname{Tr} \mathcal{M}^{-1}(q)=H(q)$ which is the character of the module for $N=-1$ is the character of the other module of this $J^{+}$subalgebra of $\mathrm{SU}(2)_{1}$, which has as the vacuum the fundamental representation. Moreover one can get to $H(q)$ for $\operatorname{Tr} \mathcal{M}(q)$ by inserting a line operator (as was shown in $[6]$ and we will now review).

Insertion of line operators. With reference to the quiver in figure 3 , the $N=+1$ traces $\left\langle X_{\gamma}\right\rangle_{+1}$ were computed $^{17}$ in [6]; there it was shown that they satisfy a three-term recursion in the charge $\gamma$ of the inserted line operator. This recursion relation is a manifestation of the Verlinde algebra of the underlying 2d CFT. Explicitly [6]

$$
\left\langle X_{m e_{1}+n e_{2}}\right\rangle_{N=+1}=\frac{(-1)^{m+n}}{(q)_{\infty}^{4}} q^{\left(m^{2}+n^{2}-m n\right) / 2} G_{m-n}(q)
$$

where $G_{\ell}(q)$ is the Rogers-Ramunjan function

$$
G_{\ell}(q):=\sum_{k \geq 0} \frac{q^{k^{2}+\ell k}}{(q)_{k}}
$$

which for $\ell \geq 0, G_{\ell}(q)$ may be written as a sum of (at most) $\ell$ theta-functions $^{18}$

$$
G_{\ell}(q)=\frac{1}{(q)_{\infty}} \sum_{s=0}^{\ell}\left[\begin{array}{l}
\ell \\
s
\end{array}\right]_{q} q^{2 s(s-\ell)} \theta\left(q^{3+4 s-2 \ell} ; q^{5}\right), \quad \ell \geq 0 .
$$

In particular, $G_{0}(q) \equiv G(q)$ and $G_{1}(q) \equiv H(q)$. The Garrett-Ismail-Stanton theorem ${ }^{19}$ [55] solves the Verlinde algebra three-terms recursion for $G_{\ell}(q)$ in terms of the Schur polynomials $e_{\ell}(z)$ and $d_{\ell}(z)(\ell \in \mathbb{Z})$. This allows to write $G_{\ell}(q)$ for $\ell \in \mathbb{Z}$ in a form which generalizes the r.h.s. of eqs. (7.5), (7.7)

$$
G_{\ell}(q)=\frac{e_{-\ell}(1 / q)}{\left(q, q^{4} ; q^{5}\right)_{\infty}}+\frac{d_{-\ell}(1 / q)}{\left(q^{2}, q^{3} ; q^{5}\right)_{\infty}}
$$

\footnotetext{
${ }^{17}$ In the conventions of $[6]$ the reference ray was $\theta=\pi$ instead of $\theta=0$. Hence $\left.\left\langle X_{\gamma}\right\rangle\right|_{[6]}=\left.\left\langle X_{-\gamma}\right\rangle\right|_{\text {this paper }}$.

${ }^{18} \theta$ is defined as in eq. (6.16).

${ }^{19}$ We thank Ole Warnaar for pointing out the GIS theorem to us.
} 
where the Schur polynomials $e_{\ell}(x), d_{\ell}(x)$ are defined for $\ell \geq 0$ as

$$
d_{\ell-1}(q)=\sum_{j} q^{j^{2}+j}\left[\begin{array}{c}
\ell-j-2 \\
j
\end{array}\right]_{q} \quad e_{\ell-1}(q)=\sum_{j} q^{j^{2}}\left[\begin{array}{c}
\ell-j-1 \\
j
\end{array}\right]_{q},
$$

and for $\ell<0$

$$
e_{-\ell}(1 / q)=(-1)^{\ell} q^{-\left(\begin{array}{c}
\ell \\
2
\end{array}\right)} d_{\ell-1}(q), \quad d_{-\ell}(1 / q)=-(-1)^{\ell} q^{-\left(\begin{array}{l}
\ell \\
2
\end{array}\right)} e_{\ell-1}(q) .
$$

The $N=-1$ trace with line operators insertions is computed in appendix D.2. Using the identities in appendix D.1 we get an expression in terms of partial theta functions $\psi(z, q)$

$$
\begin{aligned}
& \left\langle X_{m e_{1}+n e_{2}}\right\rangle_{N=-1}= \\
& \quad=\frac{q^{m n / 2}}{(q)_{\infty}^{2}} \sum_{k \geq 0}(-1)^{k} \frac{q^{\left(k^{2}+k+|m-k|+|n+k|\right) / 2}}{(q)_{k}} \psi\left(-q^{|m-k|}, q\right) \psi\left(-q^{|n+k|}, q\right) .
\end{aligned}
$$

This sum may be explicitly evaluated in each quadrant of $\mathbb{Z}^{2}$ in terms of Rogers-Ramanujan functions $G_{\ell}(q)$. The simplest case is $m \geq 0$ and $n \leq 0$ where one gets (see appendix D.2)

$$
\left\langle X_{m e_{1}+n e_{2}}\right\rangle_{N=-1}=q^{(m-n-n m) / 2} G_{m-n+1}(q) \quad \text { for } m \geq 0, n \leq 0,
$$

which, in particular, reduces to $\operatorname{Tr} \mathcal{M}(q)^{-1}=H(q)$ for $m=n=0$. In the other three quadrants one gets the same formula up to a finite sum

$$
\left\langle X_{m e_{1}+n e_{2}}\right\rangle_{N=-1}=q^{(m-n-n m) / 2}\left(G_{m-n+1}(q)+\text { a finite } q \text {-sum }\right) .
$$

See appendix D.2 for explicit expressions in the various cases and additional details.

The traces $\operatorname{Tr} \mathcal{M}(\boldsymbol{q})^{ \pm 2}$. For any $\mathcal{N}=2$ model and any $N$ one has the identity

$$
\operatorname{Tr} \mathcal{M}(q)^{2 N}=\sum_{\gamma \in \Gamma}\left\langle X_{\gamma}\right\rangle_{N}\left\langle X_{-\gamma}\right\rangle_{N}
$$

where $\left\langle X_{\gamma}\right\rangle_{N}$ is defined as in (7.2) (we may see the monodromy traces as norms, see section C.2). Thus for the $\left(A_{1}, A_{2}\right)$ model we may write $\operatorname{Tr} \mathcal{M}(q)^{ \pm 2}$ as a bilinear sum of Rogers-Ramanujan functions $G_{\ell}(q)$. Note that as $q \rightarrow 0$

$$
\begin{aligned}
& \left\langle X_{m e_{1}+n e_{2}}\right\rangle_{N=+1}\left\langle X_{-m e_{1}-n e_{2}}\right\rangle_{N=+1}=O\left(q^{m^{2}+n^{2}-m n+[|n-m| / 2]^{2}}\right) \\
& \left\langle X_{m e_{1}+n e_{2}}\right\rangle_{N=-1}\left\langle X_{-m e_{1}-n e_{2}}\right\rangle_{N=-1}=O\left(q^{|m|+|n|}\right),
\end{aligned}
$$

so both traces $\operatorname{Tr} \mathcal{M}(q)^{ \pm 2}$ have a nice expansion in integral non-negative powers of $q$. Checking the $q$-expansion of $\operatorname{Tr} \mathcal{M}(q)^{2}$, one is lead to the following expression

$$
\operatorname{Tr} \mathcal{M}(q)^{2}=\left(\frac{G(q)}{(q)_{\infty}^{4}}\right)^{2}\left(1-10 \sum_{k=1}^{\infty}\left(\frac{q^{5 k+1}}{1-q^{5 k+1}}-2 \frac{q^{5 k+2}}{1-q^{5 k+2}}+2 \frac{q^{5(k+1)-2}}{1-q^{5(k+1)-2}}-\frac{q^{5(k+1)-1}}{1-q^{5(k+1)-1}}\right)\right) .
$$

which can be rewritten

$$
\operatorname{Tr} \mathcal{M}(q)^{2}=\left(\frac{G(q)}{(q)_{\infty}^{4}}\right)^{2}\left(1-\left.10 \frac{\partial}{\partial \ln a} \theta\left(a, q^{5}\right)\right|_{a \rightarrow q}+\left.20 \frac{\partial}{\partial \ln a} \theta\left(a, q^{5}\right)\right|_{a \rightarrow q^{2}}\right) .
$$

Therefore we see $\operatorname{Tr} \mathcal{M}^{2}$ is $(\operatorname{Tr} \mathcal{M})^{2}$ multiplied by a modular function. This again suggests that there may be a chiral algebra associated with it. 


\section{3 $\left(A_{1}, A_{2 n}\right)$ AD theories}

Trace of $\mathcal{M}(\boldsymbol{q})^{-\mathbf{1}}$. The trace of the inverse monodromy $\operatorname{Tr} \mathcal{M}(q)^{-1}$ for the model $\left(A_{1}, A_{2 n}\right)$ (as computed from the minimal chamber) is [9]

$$
\operatorname{Tr} \mathcal{M}(q)^{-1}=(q)_{\infty}^{2 n} \sum_{\ell_{1}, \cdots, \ell_{2 n} \geq 0} \frac{q^{\sum_{i}\left(\ell_{i} \ell_{i-1}+\ell_{i}\right)}}{\prod_{i}(q)_{\ell_{i}}^{2}} .
$$

The sum in the r.h.s. is explicitly evaluated in appendix D.3; one gets

$$
\operatorname{Tr} \mathcal{M}(q)^{-1}=\frac{1}{\prod_{i=0}^{n-1}\left(q^{2 i+2} ; q^{2 n+3}\right)\left(q^{2 n+1-2 i} ; q^{2 n+3}\right)} .
$$

It can be also written as

$$
\operatorname{Tr} \mathcal{M}(q)^{-1}=\frac{1}{\prod_{i=1}^{r} \theta\left(Q^{R_{i} / 2} ; Q=q^{2 n+3}\right)},
$$

where $r$ is the dimension of the Coulomb branch and $R_{i}$ are the dimension of the Coulomb branch operators.

The r.h.s. of (7.25) is the Feigin-Nakanishi-Ooguri formula for the vacuum character of the $(2,2 n+3)$ Virasoro minimal model $[56,57]$. This confirms the claim of [9] that $\operatorname{Tr} \mathcal{M}(q)^{-1}$ is equal to the vacuum character of the minimal model. The central charge of $(2,2 n+3)$ minimal model is

$$
c_{2,2 n+2}=-\frac{2 n(6 n+5)}{2 n+3} .
$$

Its effective central charge is then

$$
c_{\text {eff }}=\frac{2 n}{2 n+3}
$$

in agreement with eq. (6.85). The $2 \mathrm{~d}$ central charge is again -12 times the $4 \mathrm{~d}$ central charge, implying that this the correct $2 \mathrm{~d}$ chiral algebra constructed in $[8,53]$. It also agrees with the TQFT computation of the Schur index [58].

Trace of $\mathcal{M}(\boldsymbol{q})$. The monodromy traces $\operatorname{Tr} \mathcal{M}(q)$ for $\left(A_{1}, A_{2 n}\right)$ were computed in [6]; with the present normalizations they are

$$
\operatorname{Tr} \mathcal{M}(q)=\frac{1}{(q)_{\infty}^{4 n}} \sum_{\boldsymbol{l} \in \mathbb{Z}_{+}^{2 n-1}} \frac{q^{\boldsymbol{l} \cdot C_{2 n-1} \cdot l / 2}}{(q)_{l_{1}} \cdots(q)_{l_{2 n-1}}}
$$

where $C_{2 n-1}$ stands for the Cartan matrix of $A_{2 n-1}$. The sum in the r.h.s. is the Nahm sum associated to the pair of graphs $\left(T_{1}, A_{2 n-1}\right)$ [49]. Then its effective central charge is

$$
c_{\left(A_{1}, A_{2 n}\right)}=\frac{2 n(6 n+5)}{2 n+3} .
$$

in agreement with eq. (6.82) with $r=n, r_{G}=2 n$, and $h_{G}=2 n+1$. The sum in the r.h.s. of (7.29) is evaluated in [59] Theorem 1.2, where they are related to the Macdonald identities for the twisted Kac-Moody algebra $A^{(2)}$ as well as to the Feigin-Stoyanovsky theorem [54] we are going to discuss. 
The chiral algebra associated to $\mathcal{M}(q)$ for the $\left(A_{1}, A_{2 n}\right)$ model. The Feigin and Stoyanovsky theorem [54] describes $^{20}$ a class of $q$-sum of the form

$$
\Phi_{\mathfrak{g}}(q):=\sum_{\boldsymbol{l} \in \mathbb{Z}_{+}^{r}} \frac{q^{\boldsymbol{l} \cdot C_{\mathfrak{g}} \cdot l / 2}}{(q)_{l_{1}} \cdots(q)_{l_{2 n-1}}},
$$

where $C_{\mathfrak{g}}$ is the Cartan matrix of a simply-laced Lie algebra $\mathfrak{g} \in A D E$ of rank $r$. They consider a Cartan splitting $\mathfrak{g}=\mathfrak{n}_{-} \oplus \mathfrak{h} \oplus \mathfrak{n}_{+}$and consider the loop algebra $\widehat{\mathfrak{n}}_{+}$of the nilpotent Lie algebra $\mathfrak{n}$ seen as a subalgebra of the affine $\widehat{\mathfrak{g}}_{(1)}$ Lie algebra of type $\mathfrak{g}$ and level 1 . One takes the vacuum integrable highest weight module $\mathcal{V}$ of $\widehat{\mathfrak{g}}_{(1)}$ with vacuum vector $|v\rangle$ and focus on the cyclic submodule

$$
\mathrm{U}\left(\widehat{\mathfrak{n}}_{+}\right)|v\rangle \in \mathcal{V},
$$

where $\mathrm{U}(\cdot)$ stands for the universal covering algebra. The theorem states that $\Phi_{\mathfrak{g}}(q)$ is just the character restricted to the submodule

$$
\left.\operatorname{Tr} q^{L_{0}}\right|_{\mathrm{U}\left(\widehat{\mathfrak{n}}_{+}\right)|v\rangle}=\Phi_{\mathfrak{g}}(q)
$$

In other words, the construction described around eq. (7.9) for $\mathfrak{g}=\mathfrak{s u}(2)$, in relation with the $\left(A_{1}, A_{2}\right) \mathrm{AD}$ model, extends to all simply-laced Lie algebras $\mathfrak{g}$ (and, in fact, to other situations as well). For all $\mathfrak{g}$ the function $\Phi_{\mathfrak{g}}(q)$ is the vacuum character of the chiral algebra $\mathrm{U}\left(\widehat{\mathfrak{n}}_{+}\right)$.

In particular, for the $\left(A_{1}, A_{2 n}\right)$ AD model the chiral algebra $\mathcal{A}_{1}$, whose partition function is $\operatorname{Tr} \mathcal{M}(q)$, is generated by the upper triangular $\widehat{\mathrm{SU}}(2 n)$ currents at level 1. Again this is a highly non-trivial realization of our conjecture that the relevant characters can be viewed as characters of a $2 \mathrm{~d}$ chiral algebra.

\section{$7.4 \quad\left(A_{1}, A_{2 n+1}\right)$ AD theories}

The models $\left(A_{1}, A_{2 n+1}\right)$ have a $\mathrm{U}(1)$ flavor symmetry, enhanced to $\mathrm{SU}(2)$ for $n=1$, and a Coulomb branch of dimension $n$. The monodromy traces are function of $q$ and the flavor fugacity $y$.

Trace of $\mathcal{M}(\boldsymbol{q})^{-\mathbf{1}}$. The minimal chamber expression for the trace of the inverse monodromy of the $\left(A_{1}, A_{2 n+3}\right)$ model, as a function of $q$ and the flavor fugacity $y$, is given by

$$
\begin{aligned}
& \operatorname{Tr} \mathcal{M}(q)^{-1} \equiv \sum_{m \in \mathbb{Z}} y^{m} M(q)_{m}^{(n)}=(q)_{\infty}^{2 n} \sum_{m \in \mathbb{Z}} y^{m} \times \\
& \quad \times \sum_{\substack{k_{1}, \cdots k_{2 n+1} \geq 0 \\
\ell_{1}, \cdots, \ell_{2 n+1} \geq 0}} \frac{q^{\left(\sum_{i} k_{i}\left(k_{i+1}-\ell_{i+1}+\ell_{i}+1\right)+\sum_{i} \ell_{i}\left(\ell_{i+1}+1\right)\right) / 2}}{\prod_{i}(q)_{k_{i}}(q)_{\ell_{i}}} \prod_{j=1}^{n+1} \delta_{k_{2 j-1}, \ell_{2 j-1}+|m|} \delta_{k_{2 j}, \ell_{2 j}}
\end{aligned}
$$

\footnotetext{
${ }^{20}$ In fact, at least conjecturally, their results are expected to describe the chiral algebra of all $\mathcal{N}=2$ models of the class $\left(A_{k}, G\right)$, with $G$ a simply laced Lie algebra. The relation level/rank in their theorem is the same one as in the monodromy trace case, see [6].
} 
(where we used the convention $k_{2 n+2}=\ell_{2 n+2}=0$ ). The multiple sum in the second line of the r.h.s. may be evaluated analytically, see appendix D.4. The coefficient $M(q)_{m}^{(n)}$ of $y^{m}$ has the form

$$
M(q)_{m}^{(n)}=\frac{q^{|m|}}{(q)_{\infty}\left(q^{m+1}\right)_{\infty}} \sum_{\ell \geq 0} q^{(2 n+1) \ell(\ell+m+1)} \alpha(|m|)_{\ell},
$$

where the Bailey coefficients $\alpha(|m|)_{\ell}$ are given by finite sums, see appendix D.4 for explicit formulae and alternative expressions.

Trace of $\mathcal{M}(\boldsymbol{q})$. The argument in section 9.8.1 of [6] yields for the trace $\operatorname{Tr} \mathcal{M}(q)$ of the $\left(A_{1}, A_{2 n+1}\right)$ model, seen as a function of $q$ and $y$,

$$
\operatorname{Tr} \mathcal{M}(q)=\frac{1}{(q)_{\infty}^{4 n+1}} \sum_{k \in \mathbb{Z}} y^{k} q^{(n+1) k^{2} / 2}\left(\sum_{l \in \mathbb{Z}_{+}^{2 n}} \frac{q^{l \cdot C_{2 n} \cdot l / 2-k \sum_{i}(-1)^{i} l_{i}}}{(q)_{l_{1}} \cdots(q)_{l_{2 n}}}\right),
$$

with $C_{2 n}$ the Cartan matrix of $A_{2 n}$. Each sum in the large parenthesis belongs to the Nahm class for the pair of graphs $\left(T_{1}, A_{2 n}\right)$. For $k=0,1$ these sums have been evaluated in [59] (see their Theorem 2.3). The effective central charge is then

$$
c_{\left(A_{1}, A_{2 n+1}\right)}=\frac{2\left(3 n^{2}+5 n+1\right)}{n+2},
$$

in agreement with section 6.5.

As before, the chiral algebra $\mathcal{A}_{1}$ for the $\left(A_{1}, A_{2 n+1}\right)$ model is generated by the upper triangular $\widehat{\mathrm{SU}}(2 n+1)$ currents at level 1. Moreover, according to Fortin et al. [60] this is given by the character of $\widehat{\operatorname{Sp}}(2 n)$ at level 1 .

\subsubsection{The special $\left(A_{1}, A_{3}\right)$ model}

The model $\left(A_{1}, A_{3}\right)$ is special in three (not unrelated) respects. First the Abelian flavor symmetry $\mathrm{U}(1)$ in this case enhances to $\mathrm{SU}(2)$. Second, since $\mathfrak{s u}(4) \simeq \mathfrak{s o}(4)$ this model is equivalent to $\left(A_{1}, D_{3}\right) \mathrm{AD}$, and hence enjoys the special properties which single out the type $D$ Argyres-Douglas models from their type $A, E$ brothers. Third, it the $H_{1}$ model in the sequence of SCFT with one-dimensional Coulomb branch and maximal flavor symmetry compatible with the dimension $\Delta$ of the Coulomb branch field. Hence, as discussed inn section 6.4, it has a $c$-saturating BPS chamber which implies the formula

$$
\operatorname{Tr} \mathcal{M}(q)=\frac{\Theta(y, q)}{(q)_{\infty}^{12 c}}
$$

for some theta-function $\Theta(y, q)$ function. The 4d SCFT central charge for this model is $c=1 / 2$. Let us show that the prediction (7.38) is correct. The simplest way is to use the identity

$$
\begin{aligned}
\Phi_{1}(z ; q) & :=\sum_{\ell_{1}, \ell_{2} \geq 0} \frac{q^{\ell_{1}^{2}+\ell_{2}^{2}-\ell_{1} \ell_{2}} z^{\ell_{2}-\ell_{1}}}{(q)_{\ell_{1}}(q)_{\ell_{2}}}= \\
& =\sum_{k=-\infty}^{+\infty} q^{k^{2}} z^{k}\left(\sum_{\ell_{1}=\max \{0,-k\}}^{\infty} \frac{q^{\ell_{1}\left(\ell_{1}+k\right)}}{(q)_{\ell_{1}}(q)_{\ell_{1}+k}}\right) \equiv \frac{1}{(q)_{\infty}} \sum_{k=-\infty}^{+\infty} q^{k^{2}} z^{k},
\end{aligned}
$$


where in the first equality we wrote $\ell_{2}=\ell_{1}+k$ and in the second one we used Cauchy's identity. Indeed, from eq. (7.36) with $n=1$

$$
\operatorname{Tr} \mathcal{M}(q)=\frac{1}{(q)_{\infty}^{5}} \sum_{k \in \mathbb{Z}} y^{k} q^{k^{2}} \Phi_{1}\left(q^{-k} ; q\right)=\frac{1}{(q)_{\infty}^{6}} \sum_{\boldsymbol{k} \in \mathbb{Z}^{2}} q^{\boldsymbol{k} \cdot C_{2} \cdot \boldsymbol{k} / 2} y^{k_{1}},
$$

i.e. we get (7.38) with $\Theta(y, q)$ the following one-parameter specialization of the $\mathrm{SU}(3)$ theta-function

$$
\begin{aligned}
\Theta(y, q) & =\theta_{\mathrm{SU}(3)}(y, 0, q) \\
\text { where } \theta_{\mathrm{SU}(3)}\left(y_{1}, y_{2}, q\right) & =\sum_{\boldsymbol{k} \in \mathbb{Z}^{2}} q^{\boldsymbol{k} \cdot C_{2} \cdot \boldsymbol{k} / 2} y_{1}^{k_{1}} y_{2}^{k_{2}} .
\end{aligned}
$$

\section{5 $\left(A_{1}, D_{2 n+1}\right)$ AD theories}

The Argyres-Douglas models $\left(A_{1}, D_{2 n+1}\right)$ have a $\mathrm{SU}(2)$ flavor symmetry with level $k=$ $8 n /(2 n+1)$; its conformal central charge is $c=n / 2$. The corresponding 2 d quantities, $c_{2 d}=-12 c$ and $k_{2 d}=-k / 2$ then satisfy the SU(2) Sugawara bound (6.56). The model has a $c$-saturating BPS chamber.

Trace of $\mathcal{M}(\boldsymbol{q})^{-\mathbf{1}}$. The traces of the inverse monodromy for the $\left(A_{1}, D_{2 n+1}\right)$ AD models have been studied in [9] where they were found to agree with the vacuum character of $\widehat{\mathrm{SU}(2)}$ at level $-4 n /(2 n+1)$ as expected from the arguments of [8] in view of the Sugawara saturation of $c_{2 d}$. Then

$$
\operatorname{Tr} \mathcal{M}(q)^{-1}=\frac{1}{(q)_{\infty}\left(y^{2} q\right)_{\infty}\left(y^{-2} q\right)_{\infty}} \sum_{m=0}^{\infty} q^{(2 n+1) m(m+1) / 2} \frac{z^{2 m+1}-z^{-(2 m+1)}}{z-z^{-1}} .
$$

Trace of $\mathcal{M}(\boldsymbol{q})$. Since these models saturate the two-dimensional SU(2) Sugawara bound, one expects that, as a function of $q$ and the flavor fugacity $z$,

$$
\operatorname{Tr} \mathcal{M}(q)=\frac{\boldsymbol{\Theta}(z, q)}{(q)_{\infty}^{12 c}}
$$

for some theta function $\Theta$. We check this prediction and identify $\Theta$ which turns out to the a one-parameter specialization of the theta-function for the $\mathrm{SU}(2 n+1)$ root lattice.

Following section 9.8.1 of [6], for all $\left(A, D_{r}\right)$ models we choose the quiver $Q$ in the form

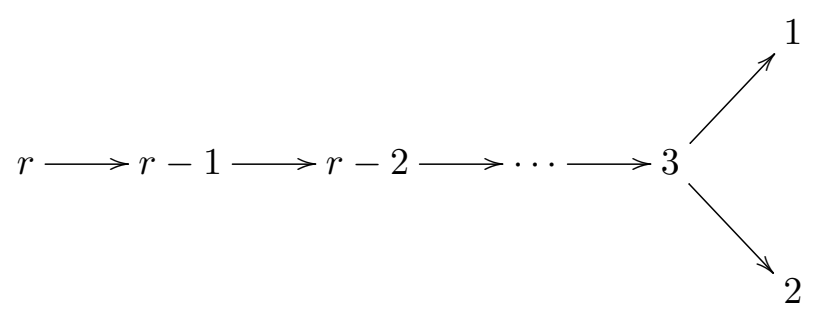

Then in the minimal chamber we have (setting $\Theta(z) \equiv \sum_{k \in \mathbb{Z}} q^{k^{2} / 2}(-z)^{k}$ )

$$
\begin{aligned}
\mathcal{M}(q)= & \frac{1}{(q)_{\infty}^{r+2[(r-1) / 2]}} \\
& \times \Theta\left(X_{e_{1}} ; q\right)\left(q^{1 / 2} X_{e_{2}}\right)_{\infty}\left(q^{1 / 2} X_{e_{1}+e_{2}-e_{3}}\right)_{\infty}\left(-q^{1 / 2} X_{e_{1}-e_{3}}\right)_{\infty}\left(-q^{1 / 2} X_{e_{2}-e_{3}}\right)_{\infty} \times \\
& \times \Theta\left(X_{e_{3}}\right)\left(q^{1 / 2} X_{e_{3}-e_{4}}\right)_{\infty} \Theta\left(X_{e_{4}}\right)\left(-q^{1 / 2} X_{e_{4}-e_{5}}\right)_{\infty} \cdots\left(-q^{1 / 2} X_{e_{r-1}-e_{r}}\right)_{\infty} \Theta\left(X_{e_{r}}\right) .
\end{aligned}
$$




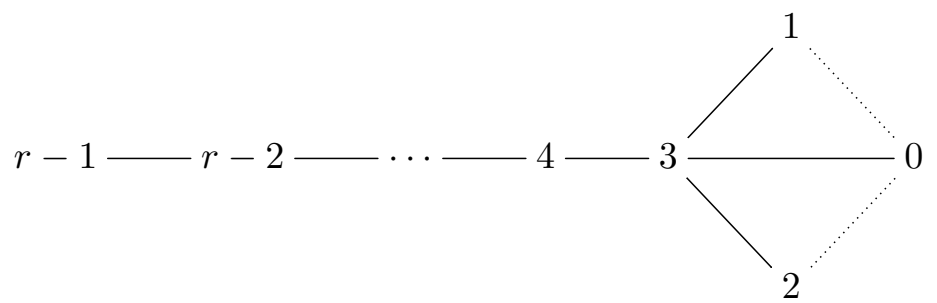

Figure 4. The bi-graph of the ideal triangulation of the punctured disk with $r$ marks on the boundary having a self-folded triangle inside an internal 2-gon.

The Dynkin quiver (7.44) is the incidence quiver of an ideal triangulation of the punctured disk with $r$ marks with a self-folded triangle inside a 2-gon having a side on the boundary [52]. Flipping the internal arc of the 2-gon we get an equivalent quiver whose Dynkin bi-graph is represented in figure 4 . We recall that a Dynkin bi-graph encodes a generalized Cartan matrix $A_{i j}$ : along the main diagonal one sets $A_{i i}=2$, while for $i \neq j$

$$
\left.A_{i j}=\#\{\text { dashed edges between } i \text { and } j\}-\# \text { solid edges between } i \text { and } j\right\},
$$

The Tits form of a bi-graph is the quadratic form $Q(\boldsymbol{m})=\boldsymbol{m} \cdot A \cdot \boldsymbol{m} / 2$. See [45] for the physical properties of the Tits forms arising from $\mathcal{N}=2$ BPS quivers. For the bi-graph in figure 4 we have

$$
2 Q(\boldsymbol{m})=m_{r-1}^{2}+\sum_{j=4}^{r-1}\left(m_{j}-m_{j-1}\right)^{2}+\frac{1}{2}\left(m_{3}-m_{0}-2 m_{1}\right)^{2}+\frac{1}{2}\left(m_{3}-m_{0}-2 m_{2}\right)^{2}+m_{0}^{2},
$$

The $\mathbb{Z}$-equivalence class of the Tits form is a mutation-invariant [45]; hence the quadratic form $Q(\boldsymbol{m})$ is $\mathbb{Z}$-equivalent to the standard Tits form of $D_{r}$. In particular it is positive definite.

Now we specialize to the odd rank case, $r=2 n+1$ (see below for $r$ even). Expanding (7.45) in the basis of $\mathbb{T}_{Q}$ and taking the trace one finds

$$
\operatorname{Tr} \mathcal{M}(q)=\frac{1}{(q)_{\infty}^{4 n+1}} \sum_{k \in \mathbb{Z}} q^{k^{2}} z^{k}\left(\sum_{m_{0}, m_{1}, \cdots, m_{2 n} \geq 0} \frac{q^{Q(\boldsymbol{m})+k\left(m_{2}-m_{1}\right)}}{(q)_{\boldsymbol{m}}}\right)
$$

The sum inside the large parenthesis is a Nahm series [49] for the positive definite 'flipped' quadratic form $Q(\boldsymbol{m})$, eq. (7.47). More generally, we may consider the Nahm sum ${ }^{21}$

$$
\Phi(x ; q):=\sum_{m_{0}, m_{1}, \cdots, m_{2 n} \geq 0} \frac{q^{Q(\boldsymbol{m})} x^{m_{1}-m_{2}}}{(q)_{\boldsymbol{m}}}
$$

Eq. (7.48) may then be written in the form

$$
\operatorname{Tr} \mathcal{M}(q)=\frac{1}{(q)_{\infty}^{4 n+1}} \sum_{k \in \mathbb{Z}} q^{k^{2}} z^{k} \Phi\left(q^{-k} ; q\right)
$$

\footnotetext{
${ }^{21}$ Here and below we use the compact notation $(q)_{\boldsymbol{m}}=\prod_{i=0}^{r-1}(q)_{m_{i}}$.
} 
The fact that for the $\left(A_{1}, D_{2 n+1}\right)$ model the $2 \mathrm{~d}$ central charge $c_{2 d}$ is saturated by the Sugawara value for its $\mathrm{SU}(2)$ current algebra, eq. (6.56), strongly suggests that the following magical identity holds

$$
\Phi(x ; q)=\frac{1}{(q)_{\infty}^{2 n-1}} \sum_{\boldsymbol{k} \in \mathbb{Z}^{2 n-1}} q^{\boldsymbol{k} \cdot C_{2 n-1} \cdot \boldsymbol{k} / 2} x^{k_{1}},
$$

where $C_{2 n-1}$ is Cartan matrix of $A_{2 n-1}$. In other words, $(q)_{\infty}^{2 n-1} \Phi(z ; q)$ is just a oneparameter specialization of the $\mathrm{SU}(2 n)$ theta-function. Thus

$$
\operatorname{Tr} \mathcal{M}(q)=\frac{1}{(q)_{\infty}^{6 n}} \sum_{\boldsymbol{k} \in \mathbb{Z}^{2 n}} z^{k_{0}} q^{k_{0}^{2}+\boldsymbol{k} \cdot C_{2 n-1} \cdot \boldsymbol{k} / 2-k_{0} k_{1}} \equiv \frac{1}{(q)_{\infty}^{6 n}} \sum_{\boldsymbol{k} \in \mathbb{Z}^{2 n}} z^{k_{1}} q^{\boldsymbol{k} \cdot C_{2 n} \cdot \boldsymbol{k} / 2}
$$

in agreement with the physical expectation in eq. (7.43), where now we see that $\boldsymbol{\Theta}$ is the one-parameter specialization of the $\mathrm{SU}(2 n+1)$ theta-function in the r.h.s. of $(7.52)$.

Specialized to $z=1, \operatorname{Tr} \mathcal{M}(q)$ is $(q)_{\infty}^{-2 n}$ times the vacuum character of $\widehat{\mathrm{SU}}(2 n+1)_{1}$, while the CNV trace

$$
\left.\operatorname{Tr} \mathcal{M}(q)\right|_{\mathrm{CNV}} \equiv \oint \frac{d z}{2 \pi i z} \operatorname{Tr} \mathcal{M}(q),
$$

is $(q)_{\infty}^{-(2 n+1)}$ times the vacuum character of $\widehat{\mathrm{SU}}(2 n)_{1}$.

The magical identity (7.51), as well as its counterpart for $\left(A_{1}, D_{r}\right)$ with $r$ even (cfr. eq. (7.59)), originally motivated by the Sugawara saturation and its implications for the monodromy $\mathcal{M}(q)$, has recently been proved by O. Warnaar $[61] .{ }^{22}$

\section{6 $\left(A_{1}, D_{2 n}\right)$ AD theories}

These Argyres-Douglas models have $\mathrm{SU}(2) \times \mathrm{U}(1)$ flavor symmetry for $n>2$, which is further enhanced to $\mathrm{SU}(2) \times \mathrm{SU}(2)$ for $n=1$ and to $\mathrm{SU}(3)$ for $n=2$ [52]. Then SCFT charges are

$$
c=(3 n-2) / 6, \quad \text { and } \quad k=2(2 n-1) / n .
$$

The corresponding $2 \mathrm{~d}$ quantities $c_{2 d}=-12 c$ and $k_{2 d}=-k / 2$ saturates the Sugawara conditions for all ${ }^{23} n \geq 2$

$$
\frac{\left(-\frac{2 n-1}{n}\right) \cdot 3}{2+\left(-\frac{2 n-1}{n}\right)}+1=-6 n+4 \equiv c_{2 d} .
$$

Note that the +1 in the above formula comes from the central charge of the chiral algebra of the current associate to the $\mathrm{U}(1)$ global symmetry. Correspondingly, we expect

$$
\operatorname{Tr} \mathcal{M}(q)=\frac{\boldsymbol{\Theta}(u, v ; q)}{(q)_{\infty}^{12 c}}
$$

for the theta-function $\boldsymbol{\Theta}(u, v ; q)$ of some positive-definite lattice $\Lambda_{n}$, specialized to two parameters i.e. the $\mathrm{SU}(2)$ fugacity $v$ and $\mathrm{U}(1)$ fugacity $u$. Below we shall show that this prediction is correct, and identify the lattice $\Lambda_{n}$ and the precise two variable specialization.

\footnotetext{
${ }^{22}$ We thank Ole Warnaar for communicating his proof to us.

${ }^{23}$ Also for $n=1$, taking into account that the quoted value of $k$ refers to the diagonal $\mathrm{SU}(2)$ subgroup.
} 
Traces of $\mathcal{M}(\boldsymbol{q})^{-\mathbf{1}}$. These traces were studied in [9] and shown to match the conjectural Schur index for these Argyres-Douglas models $[62,63]$. We refer the reader to those papers for further details.

Traces of $\mathcal{M}(\boldsymbol{q})$. Setting $r=2 n$ in eq. (7.45), and computing the trace, eq. (7.48) gets replaced by

$$
\operatorname{Tr} \mathcal{M}(q)=\frac{1}{(q)_{\infty}^{4 n-2}} \sum_{k, \ell \in \mathbb{Z}} u^{k} v^{\ell} q^{k^{2}+(2 n-1) \ell^{2}} \Phi\left(q^{-k}, q^{-\ell} ; q\right),
$$

where $\Phi(w, z ; q)$ is the following Nahm sum for the Tits form $Q(\boldsymbol{m})$ of the bi-graph in figure 4 (with $r=2 n$ )

$$
\Phi(w, z ; q)=\sum_{m_{0}, m_{1}, \cdots, m_{2 n-1} \geq 0} \frac{q^{Q(\boldsymbol{m})}}{(q)_{\boldsymbol{m}}} w^{m_{1}-m_{2}} z^{m_{1}+m_{2}+2 m_{0}+2 \sum_{j=3}^{n-1}(-1)^{j} m_{j}}
$$

The even rank counterpart to the magic identity (7.51) is the following identity

$$
\Phi(w, z ; q)=\frac{1}{(q)_{\infty}^{2 n-2}} \sum_{\boldsymbol{m} \in \mathbb{Z}^{2 n-2}} q^{\boldsymbol{m} \cdot C_{2 n-2} \cdot \boldsymbol{m} / 2} w^{m_{1}-m_{2}} z^{m_{1}+m_{2}+2 \sum_{j \geq 3}(-1)^{j} m_{j}}
$$

where $C_{2 n-2}$ is the Cartan matrix of $A_{2 n-2}$. In other words, $(q)_{\infty}^{2 n-2} \Phi(w, z ; q)$ is a twovariable specialization of the theta function for the $\mathrm{SU}(2 n-1)$ root lattice. Also this second magic identity is now a proven mathematical theorem [61].

In conclusion, we get (7.56) with

$$
\boldsymbol{\Theta}(u, v ; q)=\sum_{(k, \ell, \boldsymbol{m}) \in \mathbb{Z}^{2 n}} q^{\boldsymbol{m} \cdot C_{2 n-2} \cdot \boldsymbol{m} / 2+k^{2}+(2 n-1) \ell^{2}+k\left(m_{2}-m_{1}\right)-\ell\left\{m_{1}+m_{2}+2 \sum_{j \geq 3}(-1)^{j} m_{j}\right\}} u^{k} v^{\ell} .
$$

from which we read the lattice $\Lambda_{n}$. In particular we note that

$$
\begin{gathered}
\oint \frac{d v}{2 \pi i v} \boldsymbol{\Theta}(u, v ; q)=\text { theta-function for the } \mathrm{SU}(2 n-1) \text { lattice } \\
\oint \frac{d u d v}{(2 \pi i)^{2} u v} \boldsymbol{\Theta}(u, v ; q)=\text { theta-function for the } \mathrm{SU}(2 n-2) \text { lattice. }
\end{gathered}
$$

\section{Concluding remarks}

In this paper we have shown that an integer sequence of specializations of the $4 \mathrm{~d}$ superconformal index for $\mathcal{N}=2$ theories has interesting connections to $2 \mathrm{~d}$ chiral algebras, and moreover the characters of this algebra are captured by the traces of the powers of KS monodromy operator.

There are many things that can be done to extend the present work. The most important unanswered question is whether there always is a canonical unambiguous meaning to the trace of the powers of the monodromy operator. Moreover it would be nice to make the corresponding $2 \mathrm{~d}$ theory more physical. There is a sense in which we have succeeded in doing this: namely there is a closely related family of $2 \mathrm{~d}$ CFT's whose characters give the same integrand as the ones we get in chiral algebras. Namely the $2 \mathrm{~d}$ theories obtained 
by $\frac{N}{2} \mathrm{U}(1)_{r}$ twisted KK reduction of the 4 d theory. It would be interesting to explore the connections between these two dual perspectives.

Finally, and perhaps most importantly, we have conjectured the existence of an algebra for all $N$, but we only have circumstantial evidence for their existence (except for $N=-1$ ). It would be important to come up with an a priori definition of these algebras in general. A starting point for this is to show their existence in Lagrangian theories. For this class we managed to prove that in the extreme weak limit, there is a chiral algebra realizing the character.

\section{Acknowledgments}

We thank T. Dumitrescu, S. Gukov, A. Kirillov, K. Lee, P. Putrov, L. Rastelli, V. Stylianou, Y. Tachikawa, and O. Warnaar for useful discussions. We wish to thank the hospitality of the Simons Center for Geometry and Physics where this work was initiated during the 2015 summer workshop in mathematics and physics. SC would also like to thank the Bershadsky Visiting Fellowship while visiting the Department of Physics at Harvard University. JS would also like to thank hospitality of the Department of Physics at Harvard University. The research of CV is supported in part by NSF grant PHY-1067976. The research of JS is supported by the US Department of Energy under UCSD's contract de-sc0009919. The research of WY is supported by the Center for Mathematical Sciences and Applications at Harvard University.

\section{A Partial topological twisting and effective $2 \mathrm{~d}$ central charges}

Let us put $4 \mathrm{~d} \mathcal{N}=2$ theory on a Riemann surface $\mathcal{C}_{g}$ of genus $g$ and take the small volume limit to get an effective $2 \mathrm{~d}$ theory. In order to preserve any supersymmetry, we need to perform topological twisting along $\mathcal{C}_{g}[24,25]$. The symmetry group of the $4 \mathrm{~d} \mathcal{N}=2$ superconformal theory includes $\mathrm{SU}(2)_{1} \times \mathrm{SU}(2)_{2} \times \mathrm{SU}(2)_{R} \times \mathrm{U}(1)_{r}$, where $\mathrm{SU}(2)_{1} \times \mathrm{SU}(2)_{2}=$ $\mathrm{SO}(4)$ is the (Euclidean) Lorentz group and $\mathrm{SU}(2)_{R} \times \mathrm{U}(1)_{r}$ is the R-symmetry group. Upon dimensional reduction, the symmetry group becomes $\mathrm{SO}(2)_{E} \times \mathrm{SO}(2)_{\mathcal{C}} \times \mathrm{SU}(2)_{R} \times \mathrm{U}(1)_{r}$, where $\mathrm{SO}(2)_{E}$ and $\mathrm{SO}(2)_{\mathcal{C}}$ are the Lorentz group along the $\mathbb{R}^{2}$ and $\mathcal{C}_{g}$ respectively.

There are two linearly independent choices of twisting. We can twist with either $\mathrm{U}(1)_{r}$ or $\mathrm{SU}(2)_{R}$. If we twist by $\mathrm{U}(1)_{r}$, we get $\mathcal{N}=(0,4) \mathrm{SUSY}$ in two-dimension since $Q_{-}^{1}, Q_{-}^{2}, \tilde{Q}_{-}^{1}, \tilde{Q}_{-}^{2}$ are preserved in 2 d. Note that they all have charge $-\frac{1}{2}$ under $\operatorname{SO}(2)_{E}$. If we twist with $\mathrm{SU}(2)_{R}$, the conserved supercharges are $Q_{-}^{1}, Q_{+}^{2}, \tilde{Q}_{+}^{1}, \tilde{Q}_{-}^{2}$ so that we get $\mathcal{N}=(2,2)$. See the table 2 . Also, if we choose to do more general twisting by considering a linear combination of the two, we get $\mathcal{N}=(0,2)$ SUSY in $2 \mathrm{~d} \cdot{ }^{24}$

\footnotetext{
${ }^{24}$ Closely related twisting in the context of M5-branes wrapped on 4-cycles has been also considered in [64-66].
} 


\begin{tabular}{|c|cc|cc|cc|}
\hline$Q$ & $\mathrm{U}(1)_{E}$ & $\mathrm{U}(1)_{\mathcal{C}}$ & $\mathrm{SU}(2)_{R} \mathrm{U}(1)_{r}$ & $\mathrm{U}(1)_{R}^{(a, b)}$ & $\mathrm{U}(1)_{\mathcal{C}}^{(a, b)}$ \\
\hline$Q_{-}^{1}$ & $-\frac{1}{2}$ & $-\frac{1}{2}$ & $\frac{1}{2}$ & $\frac{1}{2}$ & $\frac{1}{2}$ & 0 \\
$Q_{+}^{1}$ & $\frac{1}{2}$ & $\frac{1}{2}$ & $\frac{1}{2}$ & $\frac{1}{2}$ & $\frac{1}{2}$ & 1 \\
$Q_{-}^{2}$ & $-\frac{1}{2}$ & $-\frac{1}{2}$ & $-\frac{1}{2}$ & $\frac{1}{2}$ & $\frac{1}{2}-a$ & $-a$ \\
$Q_{+}^{2}$ & $\frac{1}{2}$ & $\frac{1}{2}$ & $-\frac{1}{2}$ & $\frac{1}{2}$ & $\frac{1}{2}-a$ & $1-a$ \\
\hline$\tilde{Q}_{-}^{1}$ & $-\frac{1}{2}$ & $\frac{1}{2}$ & $\frac{1}{2}$ & $-\frac{1}{2}$ & $a-\frac{1}{2}$ & $a$ \\
$\tilde{Q}_{+}^{1}$ & $\frac{1}{2}$ & $-\frac{1}{2}$ & $\frac{1}{2}$ & $-\frac{1}{2}$ & $a-\frac{1}{2}$ & $a-1$ \\
$\tilde{Q}_{-}^{2}$ & $-\frac{1}{2}$ & $\frac{1}{2}$ & $-\frac{1}{2}$ & $-\frac{1}{2}$ & $-\frac{1}{2}$ & 0 \\
$\tilde{Q}_{+}^{2}$ & $\frac{1}{2}$ & $-\frac{1}{2}$ & $-\frac{1}{2}$ & $-\frac{1}{2}$ & $-\frac{1}{2}$ & -1 \\
\hline
\end{tabular}

Table 2. Supercharges of the $d=4, \mathcal{N}=2$ supersymmetry upon twisting. Here $\mathrm{U}(1)_{R}^{(a, b)}=a R+b r$ with $a+b=1$, and $\mathrm{U}(1)_{E}$ is the $2 \mathrm{~d}$ Lorentz group, and $\mathrm{U}(1)_{\mathcal{C}}$ is the Lorentz group on $\mathcal{C}$ before twisting. We see that for general twisting, only 2 supercharges $Q_{-}^{1}, \tilde{Q}_{-}^{2}$ are preserved, which are both right-moving.

\section{A.1 Twisting of the free theory ${ }^{25}$}

Let us consider the effect of twisting for the free hypermultiplet and vector multiplet. Aspects of this twisting has been already discussed in [26], and what we do here is simply to consider a linear combination of the two twists $\mathrm{U}(1)_{r}$ and $\mathrm{SU}(2)_{R}$.

One can consider a linear combination of the $\mathrm{SU}(2)_{R}$ twist and $\mathrm{U}(1)_{r}$ twist,

$$
\mathcal{C}^{(a, b)}=\mathcal{C}+a R+b r,
$$

which yields $\mathcal{N}=(0,2)$ theory. When $(a, b)=(1,0)$, we get $\mathcal{N}=(2,2)$ theory and for $(a, b)=(0,1)$ we get $\mathcal{N}=(0,4)$ theory. We also need to have $a+b=1$.

Upon the general twisting of a hypermultiplet, we get the charges as in the table 3. We see that $\left(q, \psi_{+}\right)$and $\left(\tilde{q}, \tilde{\psi}_{+}\right)$form $\mathcal{N}=(0,2)$ chiral multiplets, and become sections of $K^{\frac{a}{2}}$, where $K$ is the canonical bundle over the Riemann surface $\mathcal{C}_{g}$. Also, we get Fermi multiplets from $\left(\psi^{\dagger}\right)$ and $\left(\tilde{\psi}_{\dot{*}}^{\dagger}\right)$, which are sections of $K^{1-\frac{a}{2}}$. We expect that the twisted Lorentz group on the Riemann surface $\mathrm{U}(1)_{\mathcal{C}}^{(a, b)}$ becomes a global symmetry of the $2 \mathrm{~d}$ theory, and $\mathrm{U}(1)_{R}^{(a, b)}$ to become $R$-symmetry of the theory.

For the case of vector multiplet, we get an $\mathcal{N}=(0,2)$ vector from $\left(A_{+\dot{+}}, \lambda_{-}\right)$, and chiral multiplets from $\left(A_{+-}, \lambda_{+}\right)$in the section $\Gamma\left(\mathcal{C}_{g}, K\right)$. Also, we get another chiral multiplets from $\left(\phi, \tilde{\lambda}_{-}\right)$in $\Gamma\left(\mathcal{C}_{g}, K^{b}\right)$ and Fermi multiplets from $\left(\lambda_{-}^{\dagger}\right)$ in $\Gamma\left(\mathcal{C}_{g}, K^{a}\right)$. We summarize this in the table 5 .

We see that when $a=1, b=0,(U, \Phi)$ forms an $\mathcal{N}=(2,2)$ vector multiplet and $(\Sigma, \Theta)$ forms an $\mathcal{N}=(2,2)$ chiral multiplet in the adjoint representation, and $(Q, \Gamma)$ and $(\tilde{Q}, \tilde{\Gamma})$ form $\mathcal{N}=(2,2)$ chiral multiplets in the conjugate representations. When $a=0, b=1$, $(U, \Theta),(\Sigma, \Phi)$ form $\mathcal{N}=(0,4)$ vector, twisted hypermultiplet respectively. Also, $(Q, \tilde{Q})$ forms an hypermultiplet.

\footnotetext{
${ }^{25}$ We thank Vasilis Stylianou for the discussions and sharing his note related to this section.
} 


\begin{tabular}{|c|cc|cc|cc|}
\hline & $\mathrm{U}(1)_{E}$ & $\mathrm{U}(1)_{\mathcal{C}}$ & $\mathrm{SU}(2)_{R}$ & $\mathrm{U}(1)_{r}$ & $\mathrm{U}(1)_{R}^{(a, b)}$ & $\mathrm{U}(1)_{\mathcal{C}}^{(a, b)}$ \\
\hline$\psi_{ \pm}$ & $\pm \frac{1}{2}$ & $\pm \frac{1}{2}$ & 0 & $-\frac{1}{2}$ & $\frac{a-1}{2}$ & $\left(\frac{a}{2}, \frac{a}{2}-1\right)$ \\
$\tilde{\psi}_{ \pm}^{\dagger}$ & $\pm \frac{1}{2}$ & $\mp \frac{1}{2}$ & 0 & $\frac{1}{2}$ & $\frac{1-a}{2}$ & $\left(-\frac{a}{2}, 1-\frac{a}{2}\right)$ \\
$\psi_{ \pm}^{\dagger}$ & $\pm \frac{1}{2}$ & $\mp \frac{1}{2}$ & 0 & $\frac{1}{2}$ & $\frac{1-a}{2}$ & $\left(-\frac{a}{2}, 1-\frac{a}{2}\right)$ \\
$\tilde{\psi}_{ \pm}$ & $\pm \frac{1}{2}$ & $\pm \frac{1}{2}$ & 0 & $-\frac{1}{2}$ & $\frac{a-1}{2}$ & $\left(\frac{a}{2}, \frac{a}{2}-1\right)$ \\
\hline$q$ & 0 & 0 & $\frac{1}{2}$ & 0 & $\frac{a}{2}$ & $\frac{a}{2}$ \\
$\tilde{q}^{\dagger}$ & 0 & 0 & $-\frac{1}{2}$ & 0 & $-\frac{a}{2}$ & $-\frac{a}{2}$ \\
$q^{\dagger}$ & 0 & 0 & $-\frac{1}{2}$ & 0 & $-\frac{a}{2}$ & $-\frac{a}{2}$ \\
$\tilde{q}$ & 0 & 0 & $\frac{1}{2}$ & 0 & $\frac{a}{2}$ & $\frac{a}{2}$ \\
\hline
\end{tabular}

Table 3. Twisting hypermultiplets with both $\mathrm{SU}(2)_{R}$ and $\mathrm{U}(1)_{r}$.

\begin{tabular}{|c|cc|cc|cc|}
\hline & $\mathrm{U}(1)_{E}$ & $\mathrm{U}(1)_{\mathcal{C}}$ & $\mathrm{SU}(2)_{R}$ & $\mathrm{U}(1)_{r}$ & $\mathrm{U}(1)_{R}^{(a, b)}$ & $\mathrm{U}(1)_{\mathcal{C}}^{(a, b)}$ \\
\hline$A_{\alpha \dot{\beta}}$ & $(1,-1,0,0)$ & $(0,0,1,-1)$ & 0 & 0 & 0 & $(0,0,1,-1)$ \\
$\lambda_{ \pm}$ & $\pm \frac{1}{2}$ & $\pm \frac{1}{2}$ & $\frac{1}{2}$ & $\frac{1}{2}$ & $\frac{1}{2}$ & $(1,0)$ \\
$\tilde{\lambda}_{ \pm}$ & $\pm \frac{1}{2}$ & $\pm \frac{1}{2}$ & $-\frac{1}{2}$ & $\frac{1}{2}$ & $\frac{1}{2}-a$ & $(1-a,-a)$ \\
$\lambda_{ \pm}^{\dagger}$ & $\pm \frac{1}{2}$ & $\mp \frac{1}{2}$ & $-\frac{1}{2}$ & $-\frac{1}{2}$ & $-\frac{1}{2}$ & $(-1,0)$ \\
$\tilde{\lambda}_{ \pm}^{\dagger}$ & $\pm \frac{1}{2}$ & $\mp \frac{1}{2}$ & $\frac{1}{2}$ & $-\frac{1}{2}$ & $a-\frac{1}{2}$ & $(a-1, a)$ \\
$\phi$ & 0 & 0 & 0 & 1 & $1-a$ & $1-a$ \\
$\phi^{\dagger}$ & 0 & 0 & 0 & -1 & $a-1$ & $a-1$ \\
\hline
\end{tabular}

Table 4. Twisting vector multiplets with both $\mathrm{SU}(2)_{R}$ and $\mathrm{U}(1)_{r}$.

\begin{tabular}{|c|c|cc|cc|c|c|}
\hline superfield & components & $\mathrm{SU}(2)_{R}$ & $\mathrm{U}(1)_{r}$ & $\mathrm{U}(1)_{R_{0}}^{(a, b)}$ & $\mathrm{U}(1)_{R}$ & multiplicity & $\mathcal{C}_{g}=\mathbb{P}^{1}$ \\
\hline vector $U$ & $\left(A_{+\dot{+}}, \lambda_{-}\right)$ & $\left(0, \frac{1}{2}\right)$ & $\left(0, \frac{1}{2}\right)$ & 0 & 0 & $h^{0}\left(\mathcal{C}_{g}, \mathcal{O}\right)=1$ & 1 \\
chiral $\Sigma$ & $\left(A_{-\dot{+}}, \lambda_{\dot{+}}^{\dagger}\right)$ & $\left(0,-\frac{1}{2}\right)$ & $\left(0,-\frac{1}{2}\right)$ & 0 & 0 & $h^{0}\left(\mathcal{C}_{g}, K\right)=g$ & 0 \\
chiral $\Phi$ & $\left(\phi, \tilde{\lambda}_{+}\right)$ & $\left(0,-\frac{1}{2}\right)$ & $\left(1, \frac{1}{2}\right)$ & $2-2 a$ & $2-2 \alpha$ & $h^{0}\left(\mathcal{C}_{g}, K^{b}\right)$ & $2 a-1$ \\
Fermi $\Theta$ & $\left(\tilde{\lambda}_{-}\right)$ & $-\frac{1}{2}$ & $\frac{1}{2}$ & $2 b-1$ & $1-2 \alpha$ & $h^{0}\left(\mathcal{C}_{g}, K^{a}\right)$ & $2 b-1$ \\
\hline chiral $Q$ & $\left(q, \psi_{+}\right)$ & $\left(\frac{1}{2}, 0\right)$ & $\left(0,-\frac{1}{2}\right)$ & $1-b$ & $\alpha$ & $h^{0}\left(\mathcal{C}_{g}, K^{\frac{a}{2}}\right)$ & $b$ \\
chiral $\tilde{Q}$ & $\left(\tilde{q}, \tilde{\psi}_{+}\right)$ & $\left(\frac{1}{2}, 0\right)$ & $\left(0,-\frac{1}{2}\right)$ & $1-b$ & $\alpha$ & $h^{0}\left(\mathcal{C}_{g}, K^{\frac{a}{2}}\right)$ & $b$ \\
Fermi $\Gamma$ & $\left(\psi_{\dot{-}}^{\dagger}\right)$ & 0 & $\frac{1}{2}$ & $1-a$ & $1-\alpha$ & $h^{0}\left(\mathcal{C}_{g}, K^{1-\frac{a}{2}}\right)$ & $a-1$ \\
Fermi $\tilde{\Gamma}$ & $\left(\tilde{\psi}_{\dot{-}}^{\dagger}\right)$ & 0 & $\frac{1}{2}$ & $1-a$ & $1-\alpha$ & $h^{0}\left(\mathcal{C}_{g}, K^{1-\frac{a}{2}}\right)$ & $a-1$ \\
\hline
\end{tabular}

Table 5. Summary of general $\mathcal{N}=(0,2)$ twist of $4 \mathrm{~d} \mathcal{N}=2$ multiplets. $R_{0}=2(a R+b r)$, $R=2(\alpha R+\beta r)$ with $a+b=1, \alpha+\beta=1$. 
The Riemann-Roch theorem for $\mathcal{C}_{g}$ tells us that

$$
h^{0}\left(\mathcal{C}_{g}, K^{a}\right)-h^{0}\left(\mathcal{C}_{g}, K^{1-a}\right)=(2 a-1)(g-1)
$$

from the fact that the degree of $K$ is $2 g-2$. This can be used to determine the number of each multiplets in 2d. Especially, when $a=1$, this is nothing but Poincare duality between 1 -forms and vectors. When $a>1, g>1$, we have $h^{0}\left(\mathcal{C}_{g}, K^{1-a}\right)=0$. Hence we have

$$
h^{0}\left(\mathcal{C}_{g}, K^{a}\right)=(2 a-1)(g-1) \quad(a>1, g>1) .
$$

For the case of $g=0$, we have $h^{0}\left(\mathbb{P}^{1}, \mathcal{O}(n)\right)=n+1$ for $n \geq 0$, and 0 for otherwise. Since $K_{\mathbb{P}^{1}}=\mathcal{O}(-2)$, we have

$$
h^{0}\left(\mathbb{P}^{1}, K^{a}\right)=h^{0}\left(\mathbb{P}^{1}, \mathcal{O}(-2 a)\right)=1-2 a=2 b-1 \quad(a \leq 0, b \geq 1) .
$$

Generally, one cannot choose $a$ or $b$ to be half-integer because there is no square root of the spinor bundle. But, we can further twist the hypermultiplet by $\mathrm{U}(1)$ baryonic symmetry to split $Q$ and $\tilde{Q}$ or $\Gamma$ and $\tilde{\Gamma}$. This makes the table effectively correct with half integers by picking up only one of the chiral or Fermi multiplets between the pair.

\section{A.2 Central charge of the $2 \mathrm{~d}$ effective theory}

The central charge of the $2 \mathrm{~d} \mathcal{N}=(0,2)$ gauge theory can be computed easily from the 't Hooft anomalies of the R-symmetry as

$$
c_{R}=3 \operatorname{Tr} \gamma^{3} R^{2}, \quad c_{R}-c_{L}=\operatorname{Tr} \gamma^{3} .
$$

Once we know the effective number of $4 \mathrm{~d}$ hypermultiplets and vector multiplets, we can compute the central charges of the $2 \mathrm{~d}$ theory using the above formula and the table 5 . Now, for the twist parameter $N \equiv-2 b=2-2 a$, we get

$$
\begin{aligned}
& c_{R}=3 n_{v}\left((2 \alpha-1)^{2}(2 a-1)-1\right)+6 n_{h}(1-\alpha)^{2}(1-a), \\
& c_{L}=c_{R}-2(a-1)\left(n_{v}-n_{h}\right),
\end{aligned}
$$

where we have not specified the R-charge $\alpha$ of the chiral multiplet $Q, \tilde{Q}$ yet. If we pick $\alpha=0$, we get a central charge for the CFT on the Higgs branch of the theory. If we pick $\alpha=1$, we set the chiral adjoint $\Phi$ to have R-charge 0 , therefore we are looking at a CFT on the Coulomb branch. For $\alpha=0$, we get

$$
c_{L}^{0}=-2 N\left(n_{h}-n_{v}\right), \quad c_{R}^{0}=-3 N\left(n_{h}-n_{v}\right),
$$

and for $\alpha=1$, we get

$$
c_{L}^{1}=N\left(n_{h}+2 n_{v}\right), \quad c_{R}^{1}=3 N n_{v} .
$$


Argyres-Douglas theories. The $4 \mathrm{~d}$ central charge $(a, c)$ of the generalized ArgyresDouglas theory of type $\left(A_{k-1}, A_{n-1}\right)$ is given as $[22,67,68]$

$$
\begin{aligned}
& a(k, n)=\frac{R_{A}(k, n)}{4}+\frac{R_{B}(k, n)}{6}+\frac{5 r_{k, n}}{24}, \\
& c(k, n)=\frac{R_{B}(k, n)}{3}+\frac{r_{k, n}}{6},
\end{aligned}
$$

where

$$
\begin{aligned}
& R_{A}(k, n)=\sum_{i=m+2+\lfloor j(l-1) / k\rfloor}^{l m}\left(\frac{i k-l j}{k+k m-j}-1\right), \\
& R_{B}(k, n)=\frac{(k-1)(n-1) n k}{4(n+k)}
\end{aligned}
$$

with $n=k m+j$ and $r(k, n)$ being the dimension of the Coulomb branch of the theory. When $k$ and $n$ are coprime, the central charges are given by

$$
\begin{aligned}
& a(k, n)=\frac{(n-1)(k-1)(4 k n+4 n+4 k-1)}{48(n+k)}, \\
& c(k, n)=\frac{(n-1)(k-1)(n k+k+n)}{12(n+k)} .
\end{aligned}
$$

We can covariantize the above expression by writing $h_{G_{1}}^{\vee}=n, h_{G_{2}}^{\vee}=k$ and $k-1=\operatorname{rank}\left(G_{1}\right)$ and $n-1=\operatorname{rank}\left(G_{2}\right)$. From this, we can extract the effective number of hypermultiplets and vector multiplets by using

$$
n_{h}=4(2 a-c), \quad n_{v}=4(5 c-4 a) .
$$

From this data, we can compute the effective $2 d$ central charge via (A.8), (A.9). When $k$ and $n$ are coprime, we find

$$
c_{L}^{0}=\frac{-N(k-1)(n-1)}{n+k}, \quad c_{R}^{0}=\frac{-3 N(k-1)(n-1)}{2(n+k)},
$$

and

$$
c_{L}^{1}=\frac{N(k-1)(n-1)(n k+n+k)}{n+k}, \quad c_{R}^{1}=\frac{N(k-1)(n-1)(2 n k+2 n+2 k-1)}{2(n+k)} .
$$

Here we tabulate a number of examples in table 6 . We find when $N=-1, c_{L}^{1}$ coincide with the central charge of the chiral algebra of [8].

\section{B Superconformal index for Lagrangian theories}

The $4 d$ superconformal index is evaluated by a trace formula,

$$
\mathcal{I}\left(\mu_{i}\right)=\operatorname{Tr}(-1)^{F} e^{-\mu_{i} T_{i}} e^{-\beta \delta}, \quad \delta=2\left\{\mathcal{Q}, \mathcal{Q}^{\dagger}\right\},
$$




\begin{tabular}{|c|cc|cc|cc|cc|}
\hline theory & $a$ & $c$ & $n_{h}$ & $n_{v}$ & $c_{L}^{0}$ & $c_{R}^{0}$ & $c_{L}^{1}$ & $c_{R}^{1}$ \\
\hline$\left(A_{1}, A_{2}\right)$ & $43 / 120$ & $11 / 30$ & $8 / 5$ & $7 / 5$ & $-2 N / 5$ & $-3 N / 5$ & $22 N / 5$ & $21 N / 5$ \\
$\left(A_{1}, A_{3}\right)$ & $11 / 24$ & $1 / 2$ & $8 / 3$ & $5 / 3$ & $-2 N$ & $-3 N$ & $6 N$ & $5 N$ \\
$\left(A_{1}, A_{4}\right)$ & $67 / 84$ & $17 / 21$ & $24 / 7$ & $22 / 7$ & $-4 N / 7$ & $-6 N / 7$ & $68 N / 7$ & $66 N / 7$ \\
$\left(A_{1}, A_{5}\right)$ & $11 / 12$ & $23 / 24$ & $9 / 2$ & $7 / 2$ & $-2 N$ & $-3 N$ & $23 N / 2$ & $21 N / 2$ \\
\hline$\left(A_{2}, A_{2}\right)$ & $7 / 12$ & $2 / 3$ & 4 & 2 & $-4 N$ & $-3 N / 5$ & $8 N$ & $21 N / 5$ \\
$\left(A_{2}, A_{3}\right)$ & $75 / 56$ & $19 / 14$ & $40 / 7$ & $37 / 7$ & $-6 N / 7$ & $-3 N$ & $114 N / 7$ & $5 N$ \\
\hline
\end{tabular}

Table 6. $4 \mathrm{~d} / 2 \mathrm{~d}$ central charges for Argyres-Douglas theories. Here $\left(c_{L}^{0}, c_{R}^{0}\right)=\left(2\left(n_{h}-n_{v}\right), 3\left(n_{h}-\right.\right.$ $\left.\left.n_{v}\right)\right)$ and $\left(c_{L}^{1}, c_{R}^{1}\right)=\left(n_{h}+2 n_{v},-3 n_{v}\right)$. For the $N$-twist corresponding to the $\operatorname{Tr} \mathcal{M}^{N}$, multiply the expression by $-N$.

\begin{tabular}{|c|c|c|c|c|c|c|}
\hline $\mathcal{Q}$ & $\mathrm{SU}(2)_{1}$ & $\mathrm{SU}(2)_{2}$ & $\mathrm{SU}(2)_{R}$ & $\mathrm{U}(1)_{r}$ & $\delta$ & Commuting $\delta \mathrm{s}$ \\
\hline $\mathcal{Q}_{1-}$ & $-\frac{1}{2}$ & 0 & $\frac{1}{2}$ & $\frac{1}{2}$ & $\delta_{1-}=\Delta-2 j_{1}-2 R-r$ & $\delta_{2+}, \quad \tilde{\delta}_{1 \dot{+}}, \quad \tilde{\delta}_{1-}$ \\
\hline $\mathcal{Q}_{1+}$ & $\frac{1}{2}$ & 0 & $\frac{1}{2}$ & $\frac{1}{2}$ & $\delta_{1+}=\Delta+2 j_{1}-2 R-r$ & $\delta_{2-}, \quad \tilde{\delta}_{1 \dot{+}}, \quad \tilde{\delta}_{1-}$ \\
\hline $\mathcal{Q}_{2-}$ & $-\frac{1}{2}$ & 0 & $-\frac{1}{2}$ & $\frac{1}{2}$ & $\delta_{2-}=\Delta-2 j_{1}+2 R-r$ & $\delta_{1+}, \quad \tilde{\delta}_{2 \dot{+}}, \quad \tilde{\delta}_{2-}$ \\
\hline $\mathcal{Q}_{2+}$ & $\frac{1}{2}$ & 0 & $-\frac{1}{2}$ & $\frac{1}{2}$ & $\delta_{2+}=\Delta+2 j_{1}+2 R-r$ & $\delta_{1-}, \quad \tilde{\delta}_{2 \dot{+}}, \quad \tilde{\delta}_{2-}$ \\
\hline$\widetilde{\mathcal{Q}}_{1 \dot{-}}$ & 0 & $-\frac{1}{2}$ & $\frac{1}{2}$ & $-\frac{1}{2}$ & $\tilde{\delta}_{1 \dot{-}}=\Delta-2 j_{2}-2 R+r$ & $\tilde{\delta}_{2 \dot{+}}, \quad \delta_{1+}, \quad \delta_{1-}$ \\
\hline$\widetilde{\mathcal{Q}}_{1 \dot{+}}$ & 0 & $\frac{1}{2}$ & $\frac{1}{2}$ & $-\frac{1}{2}$ & $\tilde{\delta}_{1 \dot{+}}=\Delta+2 j_{2}-2 R+r$ & $\tilde{\delta}_{2-}, \quad \delta_{1+}, \quad \delta_{1-}$ \\
\hline$\widetilde{\mathcal{Q}}_{2-}$ & 0 & $-\frac{1}{2}$ & $-\frac{1}{2}$ & $-\frac{1}{2}$ & $\tilde{\delta}_{2-}=\Delta-2 j_{2}+2 R+r$ & $\tilde{\delta}_{1+}, \quad \delta_{2+}, \quad \delta_{2-}$ \\
\hline$\widetilde{\mathcal{Q}}_{2 \dot{+}}$ & 0 & $\frac{1}{2}$ & $-\frac{1}{2}$ & $-\frac{1}{2}$ & $\tilde{\delta}_{2 \dot{+}}=\Delta+2 j_{2}+2 R+r$ & $\tilde{\delta}_{1-}, \quad \delta_{2+}, \quad \delta_{2-}$ \\
\hline
\end{tabular}

Table 7. For each supercharge $\mathcal{Q}$, we list its quantum numbers, the associated $\delta \equiv 2\left\{\mathcal{Q}, \mathcal{Q}^{\dagger}\right\}$, and the other $\delta$ s commuting with it. Here $I=1,2$ are $\mathrm{SU}(2)_{R}$ indices and $\alpha= \pm, \dot{\alpha}= \pm$ Lorentz indices. $\Delta$ is the conformal dimension, $\left(j_{1}, j_{2}\right)$ the Cartan generators of the $\mathrm{SU}(2)_{1} \otimes \mathrm{SU}(2)_{2}$ isometry group, and $(R, r)$, the Cartan generators of the $\mathrm{SU}(2)_{R} \otimes \mathrm{U}(1)_{r}$ R-symmetry group.

where $\mathcal{Q}$ is the supercharge "with respect to which" the index is calculated and $\left\{T_{i}\right\}$ a complete set of generators that commute with $\mathcal{Q}$ and with each other.

For $4 d \mathcal{N}=2$ superconformal algebra $\mathrm{SU}(2,2 \mid 2)$, the commuting subalgebra with a single supercharge is $\mathrm{SU}(1,1 \mid 2)$, which has rank three, so the $\mathcal{N}=2$ index depends on three superconformal fugacities together with flavor fugacities. Table 7 summarizes the superconformal generators commuting with each $\mathcal{Q}$.

We use supercharge $\mathcal{Q}_{1-}$ to define the index and write it as

$$
\mathcal{I}(\rho, \sigma, \tau)=\operatorname{Tr}(-1)^{F} \rho^{\frac{1}{2} \delta_{1-}^{\sim}} \sigma^{\frac{1}{2} \delta_{2+}} \tau^{\frac{1}{2} \tilde{\delta}_{1+}} e^{-\beta \tilde{\delta}_{1-}},
$$

or in another parametrization,

$$
\mathcal{I}(p, q, t)=\operatorname{Tr}(-1)^{F} p^{j_{1}-j_{2}+r} q^{j_{1}+j_{2}+r} t^{R-r} e^{-\beta \tilde{\delta}_{1-}},
$$




\begin{tabular}{|c|c|c|c|c|c|c|}
\hline Letters & $\Delta$ & $j_{1}$ & $j_{2}$ & $R$ & $r$ & $\mathcal{I}(p, q, t)$ \\
\hline $\bar{\phi}$ & 1 & 0 & 0 & 0 & 1 & $p q / t$ \\
\hline$\lambda_{1+}$ & $\frac{3}{2}$ & $\frac{1}{2}$ & 0 & $\frac{1}{2}$ & $-\frac{1}{2}$ & $-t$ \\
\hline $\bar{\lambda}_{1 \pm}$ & $\frac{3}{2}$ & 0 & $\pm \frac{1}{2}$ & $\frac{1}{2}$ & $\frac{1}{2}$ & $-q,-p$ \\
\hline$F_{++}$ & 2 & 1 & 0 & 0 & 0 & $p q$ \\
\hline$\partial_{+-} \bar{\lambda}_{1+}+\partial_{+\dot{+}} \bar{\lambda}_{1-}=0$ & $\frac{5}{2}$ & $\frac{1}{2}$ & 0 & $\frac{1}{2}$ & $\frac{1}{2}$ & $p q$ \\
\hline$q$ & 1 & 0 & 0 & $\frac{1}{2}$ & 0 & $\sqrt{t}$ \\
\hline$\psi_{+}$ & $\frac{3}{2}$ & $\frac{1}{2}$ & 0 & 0 & $\frac{1}{2}$ & $-p q / \sqrt{t}$ \\
\hline$\partial_{+ \pm}$ & 1 & $\frac{1}{2}$ & $\pm \frac{1}{2}$ & 0 & 0 & $q, p$ \\
\hline
\end{tabular}

Table 8. Contributions to the index from "single letters". We denote by $\left(\phi, \bar{\phi}, \lambda_{I, \alpha}, \bar{\lambda}_{I \dot{\alpha}}, F_{\alpha \beta}, \bar{F}_{\dot{\alpha} \dot{\beta}}\right)$ the components of the adjoint $\mathcal{N}=2$ vector multiplet, by $\left(q, \bar{q}, \psi_{\alpha}, \bar{\psi}_{\dot{\alpha}}\right)$ the components of the $\mathcal{N}=1$ chiral multiplet, and by $\partial_{\alpha \dot{\alpha}}$ the spacetime derivatives.

and the fugacities satisfy

$$
|p|<1, \quad|q|<1, \quad|t|<1, \quad\left|z_{i}\right|=1, \quad\left|\frac{p q}{t}\right|<1 .
$$

Only states satisfying

$$
\delta_{1-} \equiv \Delta-2 j_{1}-2 R-r=0
$$

contribute to the index. ${ }^{26}$

Contribution to the index from "single letters" inside $\mathcal{N}=2$ hyper-multiplet and vector multiplet is summarized in table 8 . The single letter index for each multiplet is

$$
\begin{aligned}
f^{\frac{1}{2} H}(p, q, t) & =\frac{\sqrt{t}-\frac{p q}{\sqrt{t}}}{(1-p)(1-q)}, \\
f^{V}(p, q, t) & =-\frac{p}{1-p}-\frac{q}{1-q}+\frac{\frac{p q}{t}-t}{(1-p)(1-q)} .
\end{aligned}
$$

From the single letter index, we obtain partition function by taking so-called the Plethystic exponential, defined as

$$
\mathrm{PE}[n x]_{x}=\frac{1}{(1-x)^{n}},
$$

where $n$ is an integer and $x$ is a fugacity appear in the index. The index after taking the plethystic exponent (with respect to all the fugacities) is

$$
I^{H}(p, q, t ; a)=\mathrm{PE}\left[f^{\frac{1}{2} H}(p, q, t) \sum_{w \in R} a^{w}\right]_{p, q, t, a}=\prod_{w \in R} \prod_{m, n=0}^{\infty} \frac{\left(1-a^{w} t^{-\frac{1}{2}} p^{m+1} q^{n+1}\right)}{\left(1-a^{w} t^{\frac{1}{2}} p^{m} q^{n}\right)},
$$

for the hypermultiplet and

$$
I^{V}(p, q, t ; z)=\prod_{\alpha \in \Delta} \prod_{m=0}^{\infty}\left(1-z^{\alpha} p^{m+1}\right)\left(1-z^{\alpha} q^{m+1}\right) \prod_{m, n=0}^{\infty} \frac{1-t p^{m} q^{n} z^{\alpha}}{1-t^{-1} p^{m+1} q^{n+1} z^{\alpha}},
$$

\footnotetext{
${ }^{26}$ Our definition is slightly different from the definition given in [15], where they define the index with respect to $\tilde{\mathcal{Q}}_{1}$, , but agrees with the one given in [8].
} 
for the vector multiplet. Here we introduced the flavor fugacity $a$ for the hypermultiplet, and gauge fugacity $z$ for the vector multiplet. Here $w \in R$ denotes weights of the representation $R$ and $\Delta$ is the set of all roots (including the Cartans) of the gauge group. $z^{\alpha}$ is a short-hand notation for $z^{\alpha} \equiv \prod_{i=1}^{r} z_{i}^{\alpha_{i}}$. For example, when the gauge group is $\mathrm{SU}(2), z^{\alpha}$ can be $z^{2}, z^{-2}, 1$.

\section{The tool box for monodromy traces}

There are various techniques to compute the $\left\{\operatorname{Tr} \mathcal{M}(q)^{N}\right\}$. We quickly review some of them.

\section{C.1 $q$-hypergeometric series}

This technique [6] works for models having a finite BPS chamber i.e. a chamber whose BPS spectrum consists of just $h<\infty$ hypermultiplets, so that the KS product $M(q)^{N}$ contains finitely many factors $\left(q^{1 / 2} X_{\gamma} ; q\right)_{\infty}$. In this case the most obvious technique is to expand each BPS factor in the basis $\left\{X_{\gamma}\right\}_{\gamma \in \Gamma}$ of the quantum torus algebra $\mathbb{T}$ using the two Euler's sums

$$
\begin{aligned}
& \left( \pm q^{1 / 2} X_{\gamma} ; q\right)_{\infty}^{-1}=\sum_{n \geq 0} \frac{\left( \pm q^{1 / 2}\right)^{n}}{(q ; q)_{n}} X_{n \gamma} \\
& \left( \pm q^{1 / 2} X_{\gamma} ; q\right)_{\infty}=\sum_{n \geq 0} \frac{(\mp 1)^{n} q^{n^{2} / 2}}{(q ; q)_{n}} X_{n \gamma}
\end{aligned}
$$

Multiply all these factors with the rule (6.2), we get a (formal) expression of the form

$$
\mathcal{M}(q)^{N}=\sum_{\gamma \in \Gamma} \mu(N ; q)_{\gamma} X_{\gamma}
$$

for certain coefficient functions $\left\{\mu(N ; q)_{\gamma}\right\}_{\gamma \in \Gamma}$ which are given by multiple $q$-hypergeometric sums of the general form ${ }^{27}$

$$
\mu(N ; q)_{\gamma}=\frac{1}{(q)_{\infty}^{2 r N}} \sum_{n_{i} \in \mathbb{N}^{2 h|N|}}(-1)^{\epsilon_{i} n_{i}} \frac{q^{n_{i} A_{i j} n_{j} / 2+B_{i} n_{i} / 2}}{\prod_{i}(q)_{n_{i}}} \delta\left(\gamma, n_{i} \gamma_{i}\right)
$$

where:

- $\gamma_{i}$ is the charge of the $i$-th state in the ordered KS product which (by PCT) satisfy

$$
\gamma_{i+h}=-\gamma_{i} \quad i=1,2, \ldots, 2 h|N|
$$

- $\delta\left(\gamma, \gamma^{\prime}\right)$ is the Kronecker delta in the charge lattice $\Gamma$;

\footnotetext{
${ }^{27}$ Here and below, by $\mathbb{N}$ we mean the set of non-negative integers, including zero.
} 
- $A$ (resp. $B$ ) is a certain integral quadratic (resp. linear) form which often has a nice interpretation in terms of Cartan matrices (see [6]). Explicitly ${ }^{28}$

$$
\begin{aligned}
& N>0: \quad A_{i j} n_{i} n_{j}=\sum_{i=1}^{2 h N} n_{i}^{2}+\sum_{1 \leq i<j \leq 2 h N}\left\langle\gamma_{i}, \gamma_{j}\right\rangle n_{i} n_{j}, \quad B_{i}=0 \\
& N<0: \quad A_{i j} n_{i} n_{j}=\sum_{1 \leq i<j \leq 2 h|N|}\left\langle\gamma_{i}, \gamma_{j}\right\rangle n_{i} n_{j}, \quad B_{i} n_{i}=\sum_{i} n_{i} .
\end{aligned}
$$

- the $\epsilon_{i}=0,1$ are related to the quadratic refinement; one has $\epsilon_{i+h}=\epsilon_{i}$.

The Kontsevitch-Soibelman formula says that the functions $\left\{\mu(N ; q)_{\gamma}\right\}$ are invariant under all deformations of the $\mathcal{N}=2$ central charge $Z$ provided no BPS state phase crosses the reference ray (which we fix on the positive real axis). Since $\mathcal{M}(q)^{N+M}=\mathcal{M}(q)^{N} \cdot \mathcal{M}(q)^{M}$,

$$
\mu(N+M ; q)_{\gamma}=\sum_{\delta \in \Gamma} q^{\langle\gamma, \delta\rangle / 2} \mu(N ; q)_{\gamma-\delta} \mu(M ; q)_{\delta}, \quad M, N \in \mathbb{Z}
$$

so, in principle, we can compute all monodromy traces $\left\{\operatorname{Tr} \mathcal{M}(q)^{N}\right\}_{N \in \mathbb{Z}}$ using only the coefficient functions $\mu( \pm 1 ; q)_{\gamma}$.

The trace on the quantum torus algebra $\mathbb{T}$ is defined by the rule

$$
\operatorname{Tr} X_{\gamma}= \begin{cases}y_{\gamma} & \gamma \text { is a flavor charge } \\ 0 & \text { otherwise. }\end{cases}
$$

We recall that a charge $\gamma$ is a flavor charge iff it belongs to the radical of the Dirac form, i.e. $\langle\gamma, \nu\rangle=0$ for all $\nu \in \Gamma$. The flavor charges form a sublattice $\Gamma_{f} \subset \Gamma$ of the charge lattice. Let $\left\{e_{s}\right\}$ be a set of generators of the lattice $\Gamma$ and let $\left\{\phi_{a} \equiv \phi_{a s} e_{s}\right\}$ be a set of generators of the flavor sublattice $\Gamma_{f} \subset \Gamma$; as a matter of notation, given the flavor charge $\gamma \equiv \sum_{a} n_{a} \phi_{a}$ we write $y_{\gamma} \equiv \prod_{a} y_{a}^{n_{a}}$, where $y_{a}$ is the fugacity of the $a$-th flavor charge $\phi_{a}$. Then, for all elements $\gamma \equiv m_{s} e_{s} \in \Gamma$, we have (here $f=\operatorname{rank} \Gamma_{f} \equiv \operatorname{rank} \Gamma-2 r$ )

$$
\operatorname{Tr} X_{\gamma}=\sum_{k_{a} \in \mathbb{Z}^{f}} \delta\left(\gamma, \sum_{a} k_{a} \phi_{a}\right) \prod_{a} y_{a}^{k_{a}}=\sum_{k_{a} \in \mathbb{Z}^{f}} \int \prod_{s}\left(\frac{d x_{s}}{2 \pi i x_{s}} x_{s}^{m_{s}-k_{a} \phi_{a s}}\right) \prod_{a} y_{a}^{k_{a}} .
$$

From (C.3), (C.8) the monodromy traces, as functions of $q$ and the flavor fugacities $y_{a}$, are

$$
\operatorname{Tr} \mathcal{M}(q)^{N}=\sum_{\phi \in \Gamma_{f}} \mu(N ; q)_{\phi} y_{\phi}
$$

or, more explicitly,

$$
\frac{1}{(q)_{\infty}^{2 r N}} \sum_{k_{a} \in \mathbb{Z}^{f}} \prod_{a} y_{a}^{k_{a}} \sum_{n_{i} \in \mathbb{N}^{2 h|N|}}(-1)^{\epsilon_{i} n_{i}} \frac{q^{\left(n_{i} A_{i j} n_{j}+B_{i} n_{i}\right) / 2}}{\prod_{i}(q)_{n_{i}}} \int \prod_{s}\left(\frac{d x_{s}}{2 \pi i x_{s}} x_{s}^{n_{i} \gamma_{i s}-k_{a} \phi_{a s}}\right),
$$

where $\gamma_{i} \equiv \gamma_{i s} e_{s}\left(\gamma_{i s} \in \mathbb{Z}\right)$ is the charge vector of the $i$-th hypermultiplet.

\footnotetext{
${ }^{28}$ The ordering of the $\gamma_{i}$ for $N>0$ and $N<0$ are inverse of each other, so the off-diagonal entries of $A$ differ in sign in the two cases.
} 
Although this way of defining the monodromy traces is straightforward, and in many examples it leads to nice expressions [6], it is far from being fully satisfactory. The $q$ hypergeometric sums are seldom absolutely convergent, and even when they are one would like to have expressions which are easier to handle. When the series is not convergent, one would like to have a controlled regularization procedure. This suggests replacing sums with integrals.

\section{C.2 The Moyal approach and Hardy norms}

Although it is not necessary, for simplicity we shall assume our $\mathcal{N}=2$ theory has a BPS quiver description [39]; we fix a quiver $Q$ in the mutation class and write $B_{s t}$ for its exchange matrix and $e_{s}$ for the dimension vector which is 1 on the $s$-th node of $Q$ and zero elsewhere. To $Q$ there is associated the quantum torus algebra $\mathbb{T}_{Q}$ with commutation relations $X_{e_{s}} X_{e_{t}}=q^{B_{s t}} X_{e_{t}} X_{e_{s}}$. The quantum torus algebra $\mathbb{T}_{Q}$ is isomorphic to the algebra of functions on the corresponding classical torus endowed with the Moyal product * defined by the 2 -vector $B_{t s}$. It is more convenient to use the equivalent description as the space of holomorphic functions on the complexified classical torus $T_{Q}$ (endowed with the holomorphic Moyal product). Then

(quantum torus algebra) $\longleftrightarrow$ (holomorphic functions on $T_{Q}$ with $*$ product)

To the $s$-th node of the quiver $Q$ we attach a $\mathbb{C}^{*}$ variable $u_{s}$, the $s$-th simple fugacity. The $\left\{u_{s}\right\}$ form a global coordinate system on the torus $T_{Q}$. To the charge $\gamma=\sum_{s} n_{s} e_{s}$ we associate the function $u_{\gamma}=\prod_{s} u_{s}^{n_{s}}$ called the fugacity of $\gamma$. If $\gamma$ is a flavor charge, the corresponding function is called a flavor fugacity; in this case, to avoid confusion, we replace the symbol $u_{\gamma}$ with $y_{\gamma}$. In the basis $\left\{X_{\gamma}\right\}$ of $\mathbb{T}_{Q}$ the correspondence (C.12) simply reads

$$
X_{\gamma} \leftrightarrow u_{\gamma}
$$

In particular, under (C.12) the operator $\mathcal{M}(q)^{N}$ is mapped to the holomorphic function

$$
\mu(u)^{* N}=\sum_{\gamma \in \Gamma} \mu(N ; q)_{\gamma} u_{\gamma}=\overbrace{\mu(u) * \mu(u) * \cdots * \mu(u)}^{N \text { factors }} .
$$

The trace is then defined as the integral on the quotient of the real torus $\left|u_{s}\right|=1$ by its flavor subtorus with respect to the normalized Haar measure, i.e. we integrate the non-flavor fugacities along the unit circle at fixed values of the flavor fugacities $y_{a}$.

The function $\mu(u)$ is often easier to handle than the corresponding $q$-hypergeometric sum. $\mu(u)$ satisfies a set of functional equations, which may be used to determine it without bothering to give a precise sense to the poorly convergent $q$-series. The most basic function is the one associated via (C.12) to the half-plane KS product: given a $\mathcal{N}=2$ model, a choice of quiver $Q$, and a BPS chamber (not necessarily finite) we define its Moyal function

$$
\kappa\left(u_{s} \mid q\right)
$$


to be the holomorphic function on $T_{Q}$ which corresponds under (C.12) to the inverse KS product associated to the upper half-plane

$$
K^{-1} \equiv(q)_{\infty}^{r} \prod_{\substack{\text { BPS states with } \\ 0 \leq \arg Z_{\gamma}<\pi}}^{\curvearrowleft}\left(q^{s+1 / 2} X_{\gamma} ; q\right)_{\infty}^{-(-1)^{2 s}}
$$

where the normalization factor $(q)_{\infty}^{r}$ take into account the massless photons. Equivalently, the product is taken over all stable representations of the quiver $Q$. By definition, their charges $\gamma$ belong to the positive cone $\Gamma_{+} \subset \Gamma$ (which is a strictly convex cone). Then $K^{-1}$ belongs to the positive part of the quantum torus algebra, $\mathbb{T}_{Q}^{+} \equiv \operatorname{Span}\left\{X_{\gamma}\right\}_{\gamma \in \Gamma_{+}}$, and the function $\kappa\left(u_{s} \mid q\right)$ contains only non-negative powers of the simple fugacities $u_{i}$

$$
\kappa\left(u_{i} \mid q\right)=\sum_{n_{s} \geq 0} \kappa_{n_{s}}(q) \prod_{s} u_{s}^{n_{s}} .
$$

The KS wall-crossing formula states that $\kappa\left(u_{s} \mid q\right)$ is invariant under any change of the central charge $Z$ as long as we remain in the region of parameter space covered by the given form of the quiver. The trace of the inverse monodromy is given by

$$
\operatorname{Tr} \mathcal{M}(q)^{-1}=\int_{\substack{\text { unit } \\ \text { circle }}} d v_{r} \kappa\left(u_{s} \mid q\right) \kappa\left(1 / u_{s} \mid q\right)
$$

where the integral is over the non-flavor fugacities $v_{r}$ at fixed value of the flavor ones. $\operatorname{Tr} \mathcal{M}(q)^{-1}$ is a holomorphic function of $q$ and the flavor fugacities $y_{a}$. Hence it is uniquely determined by its restriction for $q$ real and $y_{a}$ on the unit circle. So restricted, $\operatorname{Tr} \mathcal{M}(q)^{-1}$ becomes the $H^{2}$ norm of the holomorphic (in the non flavor fugacities $v_{s}$ 's) Moyal function $\kappa$

$$
\operatorname{Tr} \mathcal{M}(q)^{-1}=\|\kappa\|_{H^{2}}^{2}=\int_{\substack{\text { unit } \\ \text { circle }}} d v_{r}\left|\kappa\left(v_{r}, y_{a} \mid q\right)\right|^{2}
$$

Hence, if at a fixed value of $q$ and the flavor fugacities, the holomorphic function $\kappa$ belongs to the Hardy space $H^{2}\left(D^{2 r}\right)$ ( $D$ being the unit disk) $\operatorname{Tr} \mathcal{M}(q)^{-1}$ exists and integration term by term in (C.19) is fully justified. It may happen that the radius of convergence in non-flavor fugacity space is exactly 1 . In this case the $H^{2}$ norm diverges but the integral on the slightly smaller circles $\left|v_{r}\right|=e^{-\epsilon}$ would converge, giving a way of regularizing the monodromy trace.

Example: pure SU(2). For SU(2) SYM the associated quantum cluster mutations [6] may be seen as a functional equation for the basic Moyal function $\kappa\left(u_{1}, u_{2} \mid q\right)$

$$
\kappa\left(u_{1}, u_{2} \mid q\right)=\kappa\left(u_{2}, u_{1} \mid q\right) \quad \text { and } \quad \kappa\left(q u_{1}, u_{2} \mid q\right)=\frac{1-q^{1 / 2} u_{1}}{1-q u_{1} u_{2}} \kappa\left(u_{1}, u_{2} \mid q\right),
$$

whose solution is essentially the inverse of the $q$-binomial coefficient

$$
\kappa\left(u_{1}, u_{2} \mid q\right)=\frac{(q)_{\infty}\left(q u_{1} u_{2}\right)_{\infty}}{\left(q^{1 / 2} u_{1}\right)_{\infty}\left(q^{1 / 2} u_{2}\right)_{\infty}}=\left(1-u_{1} u_{2}\right)^{-1}\left[\begin{array}{c}
\alpha \\
\beta
\end{array}\right]_{q}^{-1}
$$


where $q^{\alpha} \equiv q u_{1} u_{2}, q^{\beta}=q^{-1 / 2} u_{1}$. Then, for pure $\mathrm{SU}(2)$

$$
\operatorname{Tr} \mathcal{M}(q)^{-1}=\int \frac{d \theta}{2 \pi}\left(\left|\frac{(q)_{\infty}}{\left(q^{1 / 2} e^{i \theta}\right)_{\infty}}\right|^{2} \int \frac{d \phi}{2 \pi}\left|\frac{\left(q e^{i(\theta+\phi)}\right)_{\infty}}{\left(q^{1 / 2} e^{i \phi}\right)_{\infty}}\right|^{2}\right) .
$$

The internal integral is easily computed with the help of the $q$-binomial theorem

$$
\begin{aligned}
\int \frac{d \phi}{2 \pi}\left|\frac{\left(q e^{i(\theta+\phi)}\right)_{\infty}}{\left(q^{1 / 2} e^{i \phi}\right)_{\infty}}\right|^{2} & ={ }_{2} \phi_{1}\left(q^{1 / 2} e^{i \theta}, q^{1 / 2} e^{-i \theta} ; q ; q ; q\right) \equiv \\
& \equiv\left|\frac{\left(q^{1 / 2} e^{i \theta}\right)_{\infty}}{(q)_{\infty}}\right|^{2} \sum_{n \geq 0} \frac{q^{n / 2} e^{i n \theta}}{1-q^{n+1 / 2} e^{-i \theta}}
\end{aligned}
$$

so that

$$
\operatorname{Tr} \mathcal{M}(q)^{-1}=\sum_{n \geq 0} q^{n(n+1)}=\psi\left(1, q^{2}\right),
$$

where $\psi(z, q)$ is Ramunajan's partial theta function (see equation (D.3)).

\section{C.3 The Moyal function of the operator $\mathcal{M}(q)^{N}$}

Let $A_{i j}$ be a symmetric $m \times m$ matrix. We define the function ${ }^{29}$

$$
\begin{aligned}
f_{A}\left(z_{i} ; q\right) & :=\sum_{n_{i} \in \mathbb{N}^{m}} \frac{q^{A_{i j} n_{i} n_{j} / 2} z_{i}^{n_{i}}}{(q)_{n_{i}}}=\oint \sum_{n_{i}=-\infty}^{+\infty} q^{A_{i j} n_{i} n_{j} / 2}\left(z_{i} / w_{i}\right)^{n_{i}} \prod_{i} \frac{d w_{i}}{2 \pi i w_{i}\left(w_{i} ; q\right)_{\infty}}= \\
& =(-2 \pi \tau)^{-m / 2}(\operatorname{det} A)^{-1 / 2} \int \exp \left[-\frac{A_{i j}^{-1} t_{i} t_{j}}{4 \pi i \tau}\right] \prod_{i} \frac{d t_{i}}{\left(z_{i} e^{t_{i}} ; q\right)_{\infty}},
\end{aligned}
$$

where we set $q=e^{2 \pi i \tau}$ and used Euler's first sum and Poisson summation. In equation (C.25) sums and integrals are convergent provided the matrix $A_{i j}$ is positive definite. In facts, for $\left|z_{i}\right|<1$ the series converges absolutely under the milder condition that the quadratic form $A_{i j} n_{i} n_{j}$ is weakly semi-positive. ${ }^{30}$ For general $A$ 's one should define $f_{A}\left(z_{i} ; q\right)$ by analytic continuation e.g. by modifying in the last integral the integration contours from the straight lines $L_{i}=i \mathbb{R}+c_{i}$ to suitable $\mathcal{C}_{i}$. Series and integrals of the general form in equation (C.25) are familiar from the theory of integer partitions and the Thermodynamical Bethe Ansatz (TBA). We shall refer to a $q$-series of the above form as a Nahm sum for the quadratic form $A_{i j}$; see [49] for a survey.

The Moyal functions corresponding in the sense of (C.12) to the KS products for the BPS phase sectors $0 \leq \theta<2 \pi N$ and its inverse are of this form with $A$ as in equation (C.6) (we allow $N$ to be half-integral). More precisely,

$$
\text { Moyal function of } \mathcal{M}(q)^{N}=\frac{1}{(q)_{\infty}^{2 r N}} f_{A}\left((-1)^{\epsilon_{i}} q^{B_{i} / 2} u_{i} ; q\right) \quad N \in \frac{1}{2} \mathbb{Z} .
$$

\footnotetext{
${ }^{29}$ The integral in the first line is along circles of radius less than $1,\left|w_{i}\right|<1$, while the one in the second line is taken along straight lines $L_{i}$ parallel to the imaginary axis $i \mathbb{R}$ so that $\left|z_{i} e^{t_{i}}\right|<1$.

${ }^{30} \mathrm{~A}$ quadratic form $q: \mathbb{Z}^{n} \rightarrow \mathbb{Z}$ is said to be weakly positive (resp. weakly semi-positive) if $q(v)>0$ (resp. $q(v) \geq 0)$ for all $v \in \mathbb{N}^{n} v \neq 0$.
} 
Unless the quadratic form $A$ is weakly semi-positive, to define this Moyal function we need a prescription such as a deformation of contours $L_{i} \rightarrow \mathcal{C}_{i}$. The correct contour prescription should be dictated by the physical interpretation of $\mathcal{M}(q)^{N}$ as a relative SCFT invariant. We note that $A$ may be weakly semi-positive only for $N= \pm 1 / 2$ and in this case only if the quiver is acyclic and the chamber is minimal.

Assuming a proper contour prescription exists, the monodromy trace is then

$$
\operatorname{Tr} \mathcal{M}(q)^{N}=\frac{1}{(q)_{\infty}^{2 r N}} \oint f_{N}\left((-1)^{\epsilon_{i}} q^{B_{i} / 2} x_{s}(u, y)^{\gamma_{i s}} ; q\right) \prod_{r} \frac{d u_{r}}{2 \pi i u_{r}}
$$

where $x_{s}(u, y)$ is the $s$-th simple fugacity written as a Laurent monomial in the flavor $y_{a}$ and non-flavor $u_{r}$ fugacities. Alternatively, we may write the trace as the integral of the absolute value (for $N$ odd) or the square (for $N$ even) of the Moyal function for $\mathcal{M}(q)^{N / 2}$. (This last expression has typically better convergence properties).

\section{C.4 Asymptotic analysis of $\operatorname{Tr} \mathcal{M}(q)^{N}$ : the effective central charge $c_{\text {eff }}$}

We may view the monodromy traces as powers series in $q$

$$
\sum_{n \geq 0} a_{n}(y) q^{n}
$$

whose coefficients $a_{n}(y)$ are Laurent polynomials in the flavor fugacities $y_{a}$ with integral coefficients. Given a power series $\sum_{n \geq 0} a_{n} q^{n}$ one defines its effective central charge $c_{\text {eff }}$ as

$$
c_{\mathrm{eff}}=\frac{3}{2 \pi^{2}} \lim _{n \rightarrow \infty} \frac{\left(\log a_{n}\right)^{2}}{n},
$$

or equivalently (setting $q=e^{2 \pi i \tau}$ ) via its $\tau \rightarrow 0$ asymptotic behavior

$$
\sum_{n \geq 0} a_{n} q^{n} \simeq \exp \left(\frac{2 \pi i}{\tau} \frac{c_{\mathrm{eff}}}{24}+O(1)\right) \quad \text { as } \tau \rightarrow 0 .
$$

The name 'effective central charge' stems from the fact that, when the $q$-series (C.28) is a conformal block of a (not necessarily unitary) $2 \mathrm{~d}$ CFT, one has

$$
c_{\mathrm{eff}} \equiv c-24 \min _{i}\left(h_{i}\right)
$$

with $h_{i}, c$ the conformal weights and Virasoro central charge of the CFT; in particular, for unitary CFTs, $c_{\mathrm{eff}} \equiv c$. If there are flavor symmetries, from equation (C.29) we get a function of the flavor fugacities, $c_{\mathrm{eff}}\left(y_{a}\right)$; in this case, we define the effective central charge $c_{\text {eff }}$ as the value of this function at a (suitable) critical point $\partial_{y_{a}} c_{\text {eff }}(y)=0$.

To compute $c_{\text {eff }}$, we study the $\tau \rightarrow 0$ asymptotics of the Moyal function $f_{N}\left(z_{i} ; e^{2 \pi i \tau}\right)$ adapting the standard TBA methods [49] to our situation. Since

$$
\frac{1}{(w ; q)_{\infty}} \sim \exp \left(-\frac{1}{2 \pi i \tau} \operatorname{Li}_{2}(w)+O(1)\right) \quad \text { as } \tau \rightarrow 0
$$


the integral in the second line of equation (C.25) is

$$
\int \exp \left[-\frac{1}{2 \pi i \tau}\left(A_{i j}^{-1} t_{i} t_{j} / 2+\sum_{i} \operatorname{Li}_{2}\left(z_{i} e^{t_{i}}\right)\right)+O(1)\right] d t
$$

As $\tau \rightarrow 0$ this integral may be evaluated by saddle point. The saddle point condition is

$$
A_{i j}^{-1} t_{j}-\log \left(1-z_{i} e^{t_{i}}\right)=0
$$

i.e. setting $U_{i}=e^{t_{i}}$,

$$
U_{j}^{A_{i j}^{-1}}+z_{i} U_{i}=1
$$

Let $U_{i}=U_{i}\left(z_{j}\right)$ be the solution. As $\tau \rightarrow 0$ we have

$$
f_{N}\left(z_{i} ; e^{2 \pi i \tau}\right) \sim \exp \left[-\frac{1}{2 \pi i \tau} \sum_{i}\left(\operatorname{Li}_{2}\left(z_{i} U_{i}\right)+\frac{1}{2} \log U_{i} \log \left(1-z_{i} U_{i}\right)\right)+O(1)\right]
$$

Plugging this asymptotic expression in equation (C.27), we see that the integrals in $u_{r}$ may also be evaluated by saddle point in the $\tau \rightarrow 0$ limit, so that $c_{\mathrm{eff}}(y)$ is given by the value of the exponent at a critical point with respect to the non-flavor fugacities at fixed values of the flavor fugacities $y$. Then $c_{\text {eff }}$ is the value of the exponent extremized with respect to all fugacities (flavor and non-flavor) $x_{s}=e^{v_{s}}$. In conclusion ${ }^{31}$

$$
c_{\text {eff }}=2 r N+\frac{6}{\pi^{2}}\left(A_{i j}^{-1} t_{i} t_{j} / 2+\sum_{i} \operatorname{Li}_{2}\left(e^{\gamma_{i s} v_{s}} e^{t_{i}}\right)\right) \quad \begin{aligned}
& \text { at a critical point } \\
& \text { in the } t_{i} \text { 's and } v_{s} \text { 's. }
\end{aligned}
$$

At a critical point

$$
\left.A_{i j}^{-1} t_{i} t_{j}\right|_{\substack{\text { critical } \\ \text { point }}} \equiv \sum_{i}\left(t_{i}+\gamma_{i s} v_{s}\right) \log \left(1-e^{\gamma_{i s} v_{s}+t_{i}}\right)
$$

so $c_{\text {eff }}$ is written in terms of the Rogers dilogaritm $L(z)=\operatorname{Li}_{2}(z)+\frac{1}{2} \log (z) \log (1-z)$ as

$$
c_{\text {eff }}=2 r N+\frac{6}{\pi^{2}} \sum_{i=1}^{2 h|N|} L\left(z_{i}\right) \quad \text { where } z_{i}=e^{\gamma_{i s} v_{s}+t_{i}} \text { at the critical point. }
$$

Eq. (C.39) gives $c_{\text {eff }}$ as a critical value of a multi-valued function. On the appropriate Riemann surface on which its analytic continuation is uni-valued there are infinitely many critical points. We have to pick out the physically correct one. This is a solution to the saddle point equations having all the symmetries of the physical problem. We have various symmetries: first we have a $\mathbb{Z}_{|N|}$ replica symmetry (cfr. section...) which acts on the above variables as $t_{i} \rightarrow t_{i+2 h}$. Then in a $\mathbb{Z}_{|N|}$ symmetric critical points the $t_{i}$ depend on $i$ only $\bmod 2 h$. Next we have the CPT symmetry which acts as $t_{i} \rightarrow t_{i+h}$ while $\gamma_{i+h}=-\gamma_{i}$; the symmetric condition is $z_{i} \equiv e^{\gamma_{i s} v_{s}+t_{i}}=z_{i+h}$, i.e. $t_{i+h}=t_{i}+2 \gamma_{i s} v_{s}$. This symmetric ansatz

\footnotetext{
${ }^{31}$ The contribution $2 r N$ arises from the overall factor $(q)_{\infty}^{-2 r N}$.
} 
automatically solves the equations $\partial_{v_{s}} S\left(t_{i}, v_{s}\right)=0$ ( $S$ being the function in the parenthesis of (C.37)). The bottom line is that we have

$$
c_{\text {eff }}=2 r N+2|N| \sum_{i=1}^{h} \frac{6}{\pi^{2}} L\left(z_{i}\right),
$$

where the $z_{i}$ are the solutions to the reduced Nahm equations

$$
z_{i}^{2}=\left(1-z_{j}\right)^{C_{i j}^{ \pm}}
$$

where the symmetric integral matrix $C_{i j}^{ \pm}$is the first principal $h \times h$ minor of the $2 h|N| \times$ $2 h|N|$ matrix $2 A_{i j}$ which depends only on the sign \pm of $N$. One has

$$
C_{i j}^{-}=2 \delta_{i j}-C_{i j}^{+} .
$$

We stress that for $|N|$ large the corresponding $q$-hypergeometric series (C.4) would not converge and so our monodromy operator and traces are implicitly defined through a suitable deformation of the integration contours $\mathcal{C}_{i}$ consistent with the CPT symmetry (i.e. $\mathcal{C}_{i+h}=\mathcal{C}_{i}$ ). Then eqs. (C.40), (C.41) give the physically correct $c_{\text {eff }}$ independently of the details of the precise contour prescription required to define $\mathcal{M}(q)^{N}$, provided only such a prescription exists. Thus $c_{\text {eff }}$ is a robust invariant of the monodromy traces which does not suffer ambiguities in its definition. However, from (C.40) we see that only the values of $c_{\text {eff }}$ for $N= \pm 1$ yield independent information.

Example 1: $\left(\boldsymbol{G}, \boldsymbol{A}_{1}\right)$ models with $\boldsymbol{N}>\mathbf{0}$. In this case the equations (C.41) take the form

$$
z_{i}^{2}=\left(1-z_{j}\right)^{C_{i j}}
$$

where $C_{i j}$ the Cartan matrix of the simply-laced Lie algebra $G$. Writing $z_{i}=w_{j}^{-C_{i j}}$, the equations take the form

$$
1+w_{j}^{2 \delta_{i j}-C_{i j}}=w_{i}^{2} .
$$

The equations (C.43) have a unique solution with $0<z_{i}<1$ which corresponds to the vacuum character of the $2 \mathrm{~d}$ coset $\mathrm{CFT} G_{2} / \mathrm{U}(1)[49,51]$. Then $6 \sum_{i} L\left(z_{i}\right) / \pi^{2}$ is the central charge of the $G_{2} / \mathrm{U}(1)$ coset CFT, and

$$
c_{\mathrm{eff}}=2 N\left(r+\frac{r_{G} h_{G}}{h_{G}+2}\right),
$$

where $r_{G}, h_{G}$ are the rank and the Coxeter number of the Lie algebra $G$ (related to its dimension by the Coxeter formula $\left.\operatorname{dim} G=r_{G}\left(h_{G}+1\right)\right)$. Moreover, $2 r$ is equal to $r_{G}$ minus the multiplicity of $h_{G} / 2$ as an exponent of $G$.

Example 2: $\left(\boldsymbol{G}, \boldsymbol{A}_{1}\right)$ models with $\boldsymbol{N}<0$. In this case the equations (C.41) take the form

$$
z_{i}^{2}=\left(1-z_{j}\right)^{2 \delta_{i j}-C_{i j}}
$$

Setting $z_{i}=-w_{j}^{2 \delta_{i j}-C_{i j}}$ we get back the Nahm equations (C.44). For $N<0$ we are interested in a different solution of these equation; nevertheless we may find it using the 
same Lie theoretic methods as in the original case [49]. For instance, for $G=A_{2 \ell}$ the unique solution of these equations with $0<z_{j}<1$ is

$$
z_{j}=-\frac{\sin [(j+2) \pi(k+1) /(2 k+3)] \sin [j \pi(k+1) /(2 k+3)]}{(\sin [\pi(k+1) /(2 k+3)])^{2}} .
$$

We recall the following special case of an identity by Kirillov [69]

$$
\begin{aligned}
\frac{48}{\pi^{2}} \sum_{k=1}^{\ell} L & \left(-\frac{\sin [(k+2) \pi(\ell+1) /(2 \ell+3)] \sin [k \pi(\ell+1) /(2 \ell+3)]}{\sin ^{2}[\pi(\ell+1) /(2 \ell+3)]}\right)= \\
& =4 \ell+\frac{3(2 \ell+1)}{2 \ell+3}-1
\end{aligned}
$$

so that, for $\left(A_{2 \ell}, A_{1}\right)$ and $N<0$

$$
c_{\text {eff }}=\frac{2|N| \ell}{2 \ell+3} .
$$

The effective central charge of the $(p, q)$ Virasoro minimal model is

$$
c_{\mathrm{eff}}(p, q)=c-\min _{r, s} h_{r, s}=1-\frac{6}{p q} .
$$

Taking $p=2$ and $q=2 \ell+3$, we get the effective central charge (C.49) for $N=-1$.

Example 3: $\left(G, G^{\prime}\right)$ models with $N>0$. Again we reduce the saddle point conditions to systems of algebraic equations already studied in a related context by Nahm [49]. Using his results, one obtains the following formula

$$
c_{\mathrm{eff}}=2 N r+N \frac{r_{G} r_{G^{\prime}} h_{G} h_{G^{\prime}}}{h_{G}+h_{G^{\prime}}} .
$$

which has been checked explicitly for $\left(G, A_{2}\right)$.

The general relation: $\boldsymbol{N}<\mathbf{0}$ versus $\boldsymbol{N}>\mathbf{0}$. The relation between $N>0$ and $N<0$ traces found in the $\left(G, A_{1}\right)$ example may be generalized to arbitrary $\mathcal{N}=2$ models (having a finite chamber). For simplicity, we write $C_{i j}$ for $C_{i j}^{+}$even if, in general, it is not a Cartan matrix in the Kac sense. Then, writing

$$
z_{i}= \begin{cases}w_{j}^{-C_{i j}} & N>0 \\ -w_{j}^{2 \delta_{i j}-C_{i j}} & N<0\end{cases}
$$

we end up for both signs with equation (C.44). Using that equation, we have

$$
\frac{6}{\pi^{2}} \sum_{i=1}^{h} L\left(z_{i}\right)=\left\{\begin{aligned}
\frac{6}{\pi^{2}} \sum_{i} L\left(w_{j}^{-C_{i j}}\right) & =\frac{6}{\pi^{2}} \sum_{i} L\left(1-w_{i}^{-2}\right)=h-\frac{6}{\pi^{2}} \sum_{i} L\left(w_{i}^{-2}\right) \\
\frac{6}{\pi^{2}} \sum_{i} L\left(-w_{j}^{2 \delta_{i j}-C_{i j}}\right) & =\frac{6}{\pi^{2}} \sum_{i} L\left(1-w_{i}^{2}\right)=-h+\frac{6}{\pi^{2}} \sum_{i} L\left(w_{i}^{-2}\right),
\end{aligned}\right.
$$


where we used the two functional equations for the Rogers dilogarithm

$$
L(x)+L(1-x)=\frac{\pi^{2}}{6}, \quad L(x)+L\left(x^{-1}\right)=\frac{\pi^{2}}{3} .
$$

Hence, as a function of the $w_{i}$ 's the central charge for $N<0$ is given by minus the expression valid for $N>0$. However, the $w_{i}$ 's for $N<0$ correspond to a different solution to eqs. (C.44) than the $w_{i}$ 's for $N>0$. In other words, in the two cases the total set of saddle points are the same, but switching the sign of the exponent we interchange the most dominating point with the less dominating one. Now, assuming the $N=1$ trace to correspond to a unitary CFT character, the set of all saddle point values is given in terms of this $2 \mathrm{~d}$ CFT as

$$
\left\{c_{\text {eff }}\right\}_{\substack{\text { all saddle } \\ \text { points }}}=\left\{c-24 h_{i} \bmod 24 \mid h_{i} \text { the dimension of a Virasoro primary }\right\}
$$

Then the sum of $c_{\text {eff }}$ for $N=+1$ and $N=-1$ is 24 times the dimension of an operator in the $2 \mathrm{~d}$ unitary CFT.

\section{Some $q$-hypergeometric identities}

For the benefit of the reader, in this appendix we collect various identities we used in the main body of the paper and sketch the proof of some of them.

\section{D.1 Expansion of $1 / \Theta$ in Ramanujan's partial thetas}

In section 4.3 .1 we introduced a function $\Xi(z ; q)$ which differs from the inverse of a Jacobi theta function only by an overall $(q)_{\infty}^{3}$ factor

$$
\Xi(z ; q):=\frac{(q)_{\infty}^{2}}{\left(q^{1 / 2} z ; q\right)_{\infty}\left(q^{1 / 2} z^{-1} ; q\right)_{\infty}} \equiv \frac{(q)_{\infty}^{3}}{\Theta(-z ; q)}
$$

(here $\left.\Theta(z ; q)=\sum_{n \in \mathbb{Z}} q^{n^{2} / 2} z^{n}\right)$ and used the identity

$$
\Xi(z ; q)=\sum_{m \in \mathbb{Z}} z^{m} q^{|m| / 2} \psi\left(-q^{|m|}, q\right),
$$

where $\psi(z, q)$ is the Ramanujan partial theta function (see [70] section 6.4)

$$
\psi(z, q)=\sum_{n=0}^{\infty} z^{n} q^{n(n+1) / 2}
$$

(in a partial theta function the sum is over the non-negative integers rather than all integers as in a complete theta function). We present two proofs of identity (D.2) since elsewhere we use some of the intermediate identities of both proofs.

First proof. From Euler's first sum one has

$$
\frac{1}{\left(q^{1 / 2} z ; q\right)_{\infty}\left(q^{1 / 2} z^{-1} ; q\right)_{\infty}}=\sum_{m, n \geq 0} \frac{q^{(m+n) / 2} z^{m-n}}{(q)_{m}(q)_{n}}=\sum_{m \in \mathbb{Z}} z^{m} q^{|m| / 2} \sum_{n \geq 0} \frac{q^{n}}{(q)_{n}(q)_{n+|m|}}
$$

The internal sums in the r.h.s. may be computed using the identity in Entry 6.3.1 of Ramunjan's lost notebook (see [70] p.115) specialized to $a=-1$ and with $b=-z$ : 
Entry 6.3.1. For all complex $z$

$$
\sum_{n=0}^{\infty} \frac{q^{n}}{(q)_{n}(z q ; q)_{n}}=\frac{1}{(q)_{\infty}(z q ; q)_{\infty}} \sum_{m=0}^{\infty}(-z)^{m} q^{m(m+1) / 2} \equiv \frac{\psi(-z, q)}{(q)_{\infty}(z q ; q)_{\infty}}
$$

Then for all integers $m$ one has

$$
\begin{aligned}
\sum_{n \geq 0} \frac{q^{n}}{(q)_{n}(q)_{n+|m|}} & =\frac{1}{(q ; q)_{|m|}} \sum_{n \geq 0} \frac{q^{n}}{(q)_{n}\left(q^{|m|+1} ; q\right)_{n}}=\quad \text { [by equation (D.5)] } \\
& =\frac{\psi\left(-q^{|m|}, q\right)}{(q)_{\infty}(q ; q)_{|m|}\left(q^{|m|+1} ; q\right)_{\infty}} \equiv \frac{\psi\left(-q^{|m|}, q\right)}{(q)_{\infty}^{2}}
\end{aligned}
$$

Second proof. We recall Entry 12.2.2 of Ramanujan's lost notebook (see [71] p.264) which expresses $\Xi(z, q)$ as a bilateral Lambert series

$$
\Xi(z, q)=\sum_{k \in \mathbb{Z}}(-1)^{k} \frac{q^{k(k+1) / 2}}{1-z q^{k+1 / 2}} .
$$

The r.h.s. may be expanded as

$$
\begin{aligned}
& \sum_{k \geq 0}(-1)^{k} q^{k(k+1) / 2}\left[1+\frac{z q^{k+1 / 2}}{1-z q^{k+1 / 2}}+\frac{z^{-1} q^{k+1 / 2}}{1-z^{-1} q^{k+1 / 2}}\right]= \\
& \quad=\sum_{m \in \mathbb{Z}} z^{m} q^{|m| / 2} \sum_{k \geq 0}(-1)^{k} q^{k(k+1) / 2+|m| k} \equiv \sum_{m \in \mathbb{Z}} z^{m} q^{|m| / 2} \psi\left(-q^{|m|}, q\right) .
\end{aligned}
$$

\section{D.2 $\operatorname{Tr}\left[\mathcal{M}(q)^{-1} X_{\gamma}\right]$ for the $\left(A_{1}, A_{2}\right)$ model}

We wish to compute the line operator insertions in the inverse monodromy trace, $\left\langle X_{\gamma}\right\rangle_{N=-1} \equiv \operatorname{Tr}\left[\mathcal{M}(q)^{-1} X_{\gamma}\right]$ with respect to the quiver orientation in figure 3. As explained in the main body of the paper, one has

$$
\mathcal{M}(q)^{-1}=\sum_{m, n \in \mathbb{Z}}\left\langle X_{-m e_{1}-n e_{2}}\right\rangle_{N=-1} X_{m e_{1}+n e_{2}}
$$

Using the minimal chamber BPS spectrum

$$
\begin{aligned}
\mathcal{M}(q)^{-1} & =(q)_{\infty}^{2}\left(q^{1 / 2} X_{e_{1}} ; q\right)_{\infty}^{-1}\left(q^{1 / 2} X_{e_{2}} ; q\right)_{\infty}^{-1}\left(q^{1 / 2} X_{e_{1}}^{-1} ; q\right)_{\infty}^{-1}\left(q^{1 / 2} X_{e_{2}}^{-1} ; q\right)_{\infty}^{-1}= \\
& =(q)_{\infty}^{2} \sum_{a, b, c, d \geq 0} \frac{q^{(a+b+c+d) / 2}}{(q)_{a}(q)_{b}(q)_{c}(q)_{d}} q^{[a(b-d)+b c+c d] / 2} X_{(a-c) e_{1}+(b-d) e_{2}} .
\end{aligned}
$$

Comparing with equation (D.9) we have

$$
\left\langle X_{m e_{1}+n e_{2}}\right\rangle_{N=-1}=(q)_{\infty}^{2} \sum_{a, b, c, d \geq 0} \frac{q^{(a+b+c+d) / 2}}{(q)_{a}(q)_{b}(q)_{c}(q)_{d}} q^{[a(b-d)+b c+c d] / 2} \delta_{-m, a-c} \delta_{-n, b-d}
$$


From this expression it is manifest that

$$
\left\langle X_{-m e_{1}-n e_{2}}\right\rangle_{N=-1}=\left\langle X_{n e_{1}+m e_{2}}\right\rangle_{N=-1}
$$

so it suffices to consider three cases: i) $m \geq 0, n \leq 0$, ii) $m, n \geq 0$ and iii) $m \leq 0, n \geq 0$. One finds

$$
\begin{gathered}
m \geq 0, n \leq 0: \quad\left\langle X_{m e_{1}+n e_{2}}\right\rangle_{-1}=q^{(m+|n|+|m| n) / 2} G_{m+|n|+1}(q) \\
m \geq 0, n \geq 0: \quad\left\langle X_{m e_{1}+n e_{2}}\right\rangle_{-1}=q^{(m-n-m n) / 2}\left(G_{m-n+1}(q)-\sum_{\ell=0}^{n-1} \frac{q^{\ell^{2}+\ell(m-n+1)}}{(q)_{\ell}}\right) \\
m \leq 0, n \geq 0: \quad\left\langle X_{m e_{1}+n e_{2}}\right\rangle_{-1}=q^{(-|m|-n+|m| n) / 2}\left(G_{-|m|-n+1}(q)-\sum_{\ell=0}^{n-1} \frac{q^{\ell^{2}+\ell(m-n+1)}}{(q)_{\ell}}-\right. \\
\left.-q^{-|m|(n+1)} \sum_{b=0}^{n-1} \sum_{c=0}^{n-b-1}(-1)^{c} \frac{\left(q^{b-n+1} ; q\right)_{c}}{(q)_{b}(q)_{c}} q^{c(c+1) / 2+c|m|+b}\right)
\end{gathered}
$$

so that in all cases we have

$$
\left\langle X_{m e_{1}+n e_{2}}\right\rangle_{N=-1}=q^{(m-n-m n) / 2} G_{m-n+1}(q)+\text { finite } q \text {-sum. }
$$

Note that the r.h.s. of eqs. (D.13), (D.14), (D.15) reduce to $G_{1}(q) \equiv H(q)$ for $m=n=0$. The proofs of eqs. (D.13), (D.14), (D.15) are long and tedious. We present only the simplest one (D.13); the strategy of proof for the other two is similar.

Lemma. Consider the double q-hypergeometric sum

$$
g(w, z ; q):=\sum_{k, l \geq 0} \frac{q^{k l} z^{k} w^{l}}{(q ; q)_{k}(w ; q)_{k}(q ; q)_{l}(z ; q)_{l}} .
$$

One has

$$
g(w, z ; q)=\frac{0 \phi_{1}(-; 0 ; q ; w z)}{(w ; q)_{\infty}(z ; q)_{\infty}}
$$

where ${ }_{0} \phi_{1}(-; c ; q ; z)$ is the basic hypergeometric series [72]

$$
{ }_{0} \phi_{1}(-; c ; q ; z)=\sum_{n \geq 0} \frac{q^{n(n-1)} z^{n}}{(q ; q)_{n}(c ; q)_{n}} .
$$

Note that the Rogers-Ramanujan functions $G_{\ell}(q)$ may be written as

$$
G_{\ell}(q)={ }_{0} \phi_{1}\left(-; 0 ; q ; q^{\ell+1}\right),
$$

so that whenever $z=q^{\ell} / w(\ell \in \mathbb{Z})$ the function $g(w, z ; q)$ is equal to the Rogers-Ramanujan function $G_{\ell-1}(q)$ up to the simple pre-factor $(w ; q)_{\infty}^{-1}\left(q^{\ell} / w ; q\right)_{\infty}^{-1}$. Eq. (D.13) is an immediate consequence of the Lemma. Indeed, for $m \geq 0$ and $n \leq 0$, only the terms with $c=a+m$ and $b=d+|n|$ contribute to the sum (D.11) which then reduces to

$$
(q)_{\infty}^{2} \sum_{a, d \geq 0} \frac{q^{[2 d a+2 a|n|+2 d m+m|n|+2 a+2 d+m+|n|] / 2}}{(q)_{a}(q)_{d}(q)_{a+m}(q)_{d+|n|}} \equiv(q)_{\infty}^{2} \frac{q^{(m|n|+m+|n|) / 2}}{(q ; q)_{m}(q ; q)_{|n|}} g\left(q^{|n|+1}, q^{m+1} ; q\right) .
$$

Evaluating the r.h.s. with the help of the Lemma yields equation (D.13). 
Proof of the Lemma. Repeated use of limiting forms of Heine's transformation [72]:

$$
\begin{aligned}
& \sum_{k, l \geq 0} \frac{q^{k l} z^{k} w^{l}}{(q ; q)_{k}(w ; q)_{k}(q ; q)_{l}(z ; q)_{l}}=\sum_{k \geq 0} \frac{z^{k}}{(q ; q)_{k}(w ; q)_{k}} \sum_{l \geq 0} \frac{\left(w q^{k}\right)^{l}}{(q ; q)_{l}(z ; q)_{l}}= \\
& \quad=\sum_{k \geq 0} \frac{z^{k}}{(q ; q)_{k}(w ; q)_{k}}{ }_{2} \phi_{1}\left(0,0, z ; q ; w q^{k}\right)=\sum_{k \geq 0} \frac{z^{k}}{(q ; q)_{k}(w ; q)_{k}} \frac{1}{\left(w q^{k} ; q\right)_{\infty}} 0 \phi_{1}\left(-; z ; q ; w z q^{k}\right)= \\
& \quad=\frac{1}{(w ; q)_{\infty}} \sum_{k \geq 0} \frac{z^{k}(w ; q)_{k}}{(q ; q)_{k}(w ; q)_{k}}{ }_{0} \phi_{1}\left(-; z ; q ; w z q^{k}\right)=\frac{1}{(w ; q)_{\infty}} \sum_{k \geq 0} \frac{z^{k}}{(q)_{k}} \sum_{l \geq 0} \frac{q^{l(l-1)} w^{l} z^{l} q^{k l}}{(q ; q)_{l}(z ; q)_{l}}= \\
& \quad=\frac{1}{(w ; q)_{\infty}} \sum_{l \geq 0} \frac{q^{l(l-1)} w^{l} z^{l}}{(q ; q)_{l}(z ; q)_{l}} \frac{1}{\left(z q^{l} ; q\right)_{\infty}}=\frac{1}{(w ; q)_{\infty}(z ; q)_{\infty}} \sum_{l \geq 0} \frac{q^{l(l-1)} w^{l} z^{l}}{(q ; q)_{l}(z ; q)_{l}}(z ; q)_{l}= \\
& \quad=\frac{1}{(w ; q)_{\infty}(z ; q)_{\infty}} \sum_{l \geq 0} \frac{q^{l(l-1)}(w z)^{l}}{(q ; q)_{l}}=\frac{0 \phi_{1}(-; 0 ; q ; w z)}{(w ; q)_{\infty}(z ; q)_{\infty}} .
\end{aligned}
$$

D.3 $\operatorname{Tr} \mathcal{M}(q)^{-1}$ for $\left(A_{1}, A_{2 n}\right)$ is the character of the $(2,2 n+3)$ minimal model For $n, m \in \mathbb{Z}_{+}$, we define recursively the functions $A^{(n)}(q)_{m}$

$$
\begin{aligned}
A^{(0)}(q)_{m} & =1 \\
A^{(n+1)}(q)_{m} & =(q)_{\infty} \sum_{k \geq 0} \frac{q^{k(m+1)}}{(q)_{k}^{2}} A^{(n)}(q)_{k} .
\end{aligned}
$$

For the model $\left(A_{1}, A_{2 n}\right)$ then we have

$$
\operatorname{Tr} \mathcal{M}(q)^{-1}=(q)_{\infty}^{2 n} \sum_{\ell_{1}, \cdots, \ell_{2 n} \geq 0} \frac{q^{\sum_{i}\left(\ell_{i} \ell_{i-1}+\ell_{i}\right)}}{\prod_{i}(q)_{\ell_{i}}^{2}}=A^{(2 n)}(q)_{0} .
$$

Lemma. For all $n \in \mathbb{Z}_{+}$we have

$$
A^{(2 n)}(q)_{m}=\sum_{s_{1} \geq 0} \frac{(q)_{m+s_{1}}}{(q)_{s_{1}}^{2}} q^{s_{1}\left(s_{1}+1\right)} \sum_{s_{2} \geq s_{1}} \frac{q^{s_{2}\left(s_{2}+1\right)}}{(q)_{s_{2}-s_{1}}} \sum_{s_{3} \geq s_{2}} \frac{q^{s_{3}\left(s_{3}+1\right)}}{(q)_{s_{3}-s_{2}}} \cdots \sum_{s_{n} \geq s_{n-1}} \frac{q^{s_{n}\left(s_{n}+1\right)}}{(q)_{s_{n}-s_{n-1}}} .
$$

Proof. Induction on $n$. For $n=0$ is true. Assume the results holds for $2 n$. Then

$$
\begin{aligned}
& A^{(2 n+1)}(q)_{m}=(q)_{\infty} \sum_{k \geq 0} \sum_{s_{1} \geq 0} \frac{q^{k(m+1)}}{(q)_{k}^{2}}(q)_{k+s_{1}} \frac{q^{s_{1}\left(s_{1}+1\right)}}{(q)_{s_{1}}^{2}} \sum_{s_{2} \geq s_{1}} \frac{q^{s_{2}\left(s_{2}+1\right)}}{(q)_{s_{2}-s_{1}}} \cdots \sum_{s_{n} \geq s_{n-1}} \frac{q^{s_{n}\left(s_{n}+1\right)}}{(q)_{s_{n}-s_{n-1}}}= \\
& =(q)_{\infty} \sum_{k \geq 0} \sum_{s_{1} \geq 0} \frac{q^{k(m+1)}}{(q)_{k}^{2}}\left(q^{s_{1}+1}\right)_{k} \frac{q^{s_{1}\left(s_{1}+1\right)}}{(q)_{s_{1}}} \sum_{s_{2} \geq s_{1}} \frac{q^{s_{2}\left(s_{2}+1\right)}}{(q)_{s_{2}-s_{1}}} \cdots \sum_{s_{n} \geq s_{n-1}} \frac{q^{s_{n}\left(s_{n}+1\right)}}{(q)_{s_{n}-s_{n-1}}}= \\
& =(q)_{\infty} \sum_{s_{1} \geq 0}{ }_{2} \phi_{1}\left(0, q^{s_{1}+1} ; q ; q ; q^{m+1}\right) \frac{q^{s_{1}\left(s_{1}+1\right)}}{(q)_{s_{1}}} \sum_{s_{2} \geq s_{1}} \frac{q^{s_{2}\left(s_{2}+1\right)}}{(q)_{s_{2}-s_{1}}} \cdots \sum_{s_{n} \geq s_{n-1}} \frac{q^{s_{n}\left(s_{n}+1\right)}}{(q)_{s_{n}-s_{n-1}}}= \\
& =(q)_{\infty} \sum_{s_{1} \geq 0} \frac{1}{\left(q^{m+1}\right)_{\infty}}{ }_{1} \phi_{1}\left(q^{-s_{1}} ; q ; q ; q^{m+s_{1}+2}\right) \frac{q^{s_{1}\left(s_{1}+1\right)}}{(q)_{s_{1}}} \sum_{s_{2} \geq s_{1}} \frac{q^{s_{2}\left(s_{2}+1\right)}}{(q)_{s_{2}-s_{1}}} \cdots \sum_{s_{n} \geq s_{n-1}} \frac{q^{s_{n}\left(s_{n}+1\right)}}{(q)_{s_{n}-s_{n-1}}}= \\
& =(q)_{m} \sum_{s_{1} \geq 0} \sum_{k=0}^{s_{1}}(-1)^{k} \frac{\left(q^{-s_{1}}\right)_{k}}{(q)_{k}^{2}} q^{k(k-1) / 2+k\left(m+s_{1}+2\right)} \frac{q^{s_{1}\left(s_{1}+1\right)}}{(q)_{s_{1}}} \sum_{s_{2} \geq s_{1}} \frac{q^{s_{2}\left(s_{2}+1\right)}}{(q)_{s_{2}-s_{1}}} \cdots \sum_{s_{n} \geq s_{n-1}} \frac{q^{s_{n}\left(s_{n}+1\right)}}{(q)_{s_{n}-s_{n-1}}},
\end{aligned}
$$


where we used various limiting forms of Heine's ${ }_{2} \phi_{1}$ transformation [72]. Now [72]

$$
\left(q^{-s_{1}}\right)_{k}=\frac{(q)_{s_{1}}}{(q)_{s_{1}-k}}(-1)^{k} q^{k(k-1) / 2-k s_{1}}
$$

so

$$
A^{(2 n+1)}(q)_{m}=(q)_{m} \sum_{k \geq 0} \sum_{s_{1} \geq k} \frac{q^{k(k+1)+k m+s_{1}\left(s_{1}+1\right)}}{(q)_{k}^{2}(q)_{s_{1}-k}} \sum_{s_{2} \geq s_{1}} \frac{q^{s_{2}\left(s_{2}+1\right)}}{(q)_{s_{2}-s_{1}}} \cdots
$$

Finally,

$$
\begin{aligned}
A^{(2 m+2)}(q)_{m} & \equiv(q)_{\infty} \sum_{\ell \geq 0} \frac{q^{\ell(m+1)}}{(q)_{\ell}^{2}} A^{(2 n)}(q)_{\ell}= \\
& =(q)_{\infty} \sum_{\ell \geq 0} \frac{q^{\ell(m+1)}}{(q)_{\ell}} \sum_{k \geq 0} \sum_{s_{1} \geq k} \frac{q^{k(k+1)+k \ell+s_{1}\left(s_{1}+1\right)}}{(q)_{k}^{2}(q)_{s_{1}-k}} \sum_{s_{2} \geq s_{1}} \frac{q^{s_{2}\left(s_{2}+1\right)}}{(q)_{s_{2}-s_{1}}} \cdots= \\
& =(q)_{\infty} \sum_{k \geq 0} \sum_{s_{1} \geq k} \frac{q^{k(k+1)+s_{1}\left(s_{1}+1\right)}}{(q)_{k}^{2}(q)_{s_{1}-k}} \frac{1}{\left(q^{m+k+1}\right)_{\infty}} \sum_{s_{2} \geq s_{1}} \frac{q^{s_{2}\left(s_{2}+1\right)}}{(q)_{s_{2}-s_{1}}} \cdots= \\
& =\sum_{k \geq 0} \frac{(q)_{m+k}}{(q)_{k}^{2}} q^{k(k+1)} \sum_{s_{1} \geq k} \frac{q^{s_{1}\left(s_{1}+1\right)}}{(q)_{s_{1}-k}} \sum_{s_{2} \geq s_{1}} \frac{q^{s_{2}\left(s_{2}+1\right)}}{(q)_{s_{2}-s_{1}}} \cdots
\end{aligned}
$$

which completes the proof of the Lemma.

Corollary. For $\left(A_{1}, A_{2 n}\right)$ one has

$$
\operatorname{Tr} \mathcal{M}(q)^{-1}=\prod_{n \neq 0, \pm 1}\left(1-q^{n}\right)^{-1} .
$$

Proof. Set $m=0$ in (D.24); the resulting sum for $A^{(2 n)}(q)_{0}$ is the l.h.s. of the celebrated Andrews-Gordon (AG) generalization of the Rogers-Ramanujan identities [73, 74]. The r.h.s. of (D.29) is the r.h.s. of the AG identities.

\section{D.4 $\operatorname{Tr} \mathcal{M}(q)^{-1}$ for the $\left(A_{1}, A_{2 n+1}\right)$ AD model}

One has

$$
\operatorname{Tr} \mathcal{M}(q)^{-1}=\sum_{m \in \mathbb{Z}} y^{m} M(q)_{m}^{(n)} \quad \text { with } \quad M(q)_{-m}^{(n)}=M(q)_{m}^{(n)} .
$$

The zero coefficient $M(q)_{0}^{(n)}$ in the trace of $\mathcal{M}(q)^{-1}$ in the sense of [6]; in the notation of appendix (D.3) then one has

$$
\begin{aligned}
M(q)_{0}^{(n)} & \equiv \frac{1}{(q)_{\infty}} A^{(2 n+1)}(q)_{0}= \\
& =\frac{1}{(q)_{\infty}} \sum_{s_{2 n+1} \geq s_{2 n} \geq \cdots \geq s_{1} \geq 0} \frac{q^{s_{1}\left(s_{1}+1\right)+s_{2}\left(s_{2}+1\right)+\cdots+s_{2 n+1}\left(s_{2 n+1}+1\right)}}{(q)_{s_{2 n+1}-s_{2 n}}(q)_{s_{2 n}-s_{2 n-1}} \cdots(q)_{s_{2}-s_{1}}} \frac{1}{(q)_{s_{1}}^{2}} .
\end{aligned}
$$

The expression of $M(q)_{0}^{(n)}$ for $m>0$ should be a generalization of this formula. We claim the following 
Lemma. For $m \geq 0$ the coefficient $M(q)_{m}^{(n)}$ is given by

$$
\frac{q^{m}}{(q)_{\infty}} \sum_{s_{2 n+1} \geq \cdots \geq s_{1} \geq 0} \frac{q^{s_{1}\left(s_{1}+m+1\right)+s_{2}\left(s_{2}+m+1\right)+\cdots+s_{2 n+1}\left(s_{2 n+1}+m+1\right)}}{(q)_{s_{2 n+1}-s_{2 n}}(q)_{s_{2 n}-s_{2 n-1}} \cdots(q)_{s_{2}-s_{1}}} \frac{1}{(q)_{s_{1}}(q)_{s_{1}+m}} .
$$

The proof is similar to the previous ones in this appendix and shall be omitted for brevity. Next we need the following fundamental result of Andrews ([74] Theorem 2)

Theorem (Andrews). Assume the two sequences $\left\{\alpha_{n}\right\}_{n \geq 0},\left\{\beta_{n}\right\}_{n \geq 0}$ form a Bailey pair, i.e.

$$
\beta_{n}=\sum_{r=0}^{n} \frac{\alpha_{r}}{(q)_{n-r}(a q)_{n+r}} \quad \text { for all } n \geq 0 .
$$

Then

$$
\frac{1}{(a q)_{\infty}} \sum_{n \geq 0} q^{k n^{2}} a^{k n} \alpha_{n}=\sum_{s_{k} \geq s_{k-1} \geq \cdots \geq s_{1} \geq 0} \frac{a^{s_{1}+s_{2}+\cdots+s_{k}} q^{s_{1}^{2}+s_{2}^{2}+\cdots+s_{k}^{2}}}{(q)_{s_{k}-s_{k-1}}(q)_{s_{k-1}-s_{k-2}} \cdots(q)_{s_{2}-s_{1}}} \beta_{s_{1}} .
$$

The sum in the r.h.s. of (D.32) as the same form as the one appearing in the r.h.s. of (D.34) with

$$
k=2 n+1, \quad a=q^{m+1}, \quad \beta_{s}=\frac{1}{(q)_{s}(q)_{s+m}} .
$$

To get the sequence $\left\{\alpha_{n}\right\}$ corresponding to the Bailey sequence $\left\{(q)_{n}^{-1}(q)_{n+m}^{-1}\right\}$ one uses the inversion formula of (D.33), see [74]

$$
\alpha_{n}=\left(1-a q^{2 n}\right) \sum_{j=0}^{n}(-1)^{n-j} \frac{(a q)_{n+j-1}}{(q)_{n-j}} q^{(n-j)(n-j-1) / 2} \beta_{j}
$$

Then

$$
\begin{aligned}
& M(q)_{m}^{(n)}= \\
& \quad=\frac{q^{|m|}}{(q)_{\infty}^{2}} \sum_{j, \ell \geq 0}(-1)^{\ell} \frac{1-q^{2 \ell+2 j+|m|+1}}{1-q^{|m|+1}} \frac{(q)_{\ell+2 j+|m|}}{(q)_{\ell}(q)_{j}(q)_{j+|m|}} q^{(2 n+1)(\ell+j)(\ell+j+|m|+1)+\ell(\ell-1) / 2} .
\end{aligned}
$$

Open Access. This article is distributed under the terms of the Creative Commons Attribution License (CC-BY 4.0), which permits any use, distribution and reproduction in any medium, provided the original author(s) and source are credited.

\section{References}

[1] S. Cecotti and C. Vafa, On classification of $N=2$ supersymmetric theories, Commun. Math. Phys. 158 (1993) 569 [hep-th/9211097] [INSPIRE].

[2] N. Seiberg and E. Witten, Electric-magnetic duality, monopole condensation and confinement in $N=2$ supersymmetric Yang-Mills theory, Nucl. Phys. B 426 (1994) 19 [Erratum ibid. B 430 (1994) 485] [hep-th/9407087] [INSPIRE]. 
[3] N. Seiberg and E. Witten, Monopoles, duality and chiral symmetry breaking in $N=2$ supersymmetric QCD, Nucl. Phys. B 431 (1994) 484 [hep-th/9408099] [INSPIRE].

[4] S. Cecotti and C. Vafa, $2 d$ wall-crossing, R-twisting and a supersymmetric index, arXiv: 1002.3638 [INSPIRE].

[5] M. Kontsevich and Y. Soibelman, Stability structures, motivic Donaldson-Thomas invariants and cluster transformations, arXiv:0811.2435 [INSPIRE].

[6] S. Cecotti, A. Neitzke and C. Vafa, R-twisting and 4d/2d correspondences, arXiv:1006.3435 [INSPIRE].

[7] A. Iqbal and C. Vafa, BPS degeneracies and superconformal index in diverse dimensions, Phys. Rev. D 90 (2014) 105031 [arXiv:1210.3605] [INSPIRE].

[8] C. Beem, M. Lemos, P. Liendo, W. Peelaers, L. Rastelli and B.C. van Rees, Infinite chiral symmetry in four dimensions, Commun. Math. Phys. 336 (2015) 1359 [arXiv:1312.5344] [INSPIRE].

[9] C. Cordova and S.-H. Shao, Schur indices, BPS particles and Argyres-Douglas theories, JHEP 01 (2016) 040 [arXiv: 1506.00265] [INSPIRE].

[10] C. Vafa, String vacua and orbifoldized $L-G$ models, Mod. Phys. Lett. A 4 (1989) 1169 [INSPIRE].

[11] J. Kinney, J.M. Maldacena, S. Minwalla and S. Raju, An index for 4 dimensional super conformal theories, Commun. Math. Phys. 275 (2007) 209 [hep-th/0510251] [INSPIRE].

[12] C. Romelsberger, Counting chiral primaries in $N=1, D=4$ superconformal field theories, Nucl. Phys. B 747 (2006) 329 [hep-th/0510060] [INSPIRE].

[13] E. Witten, Topological quantum field theory, Commun. Math. Phys. 117 (1988) 353 [INSPIRE].

[14] A. Gadde, L. Rastelli, S.S. Razamat and W. Yan, The 4d superconformal index from q-deformed 2d Yang-Mills, Phys. Rev. Lett. 106 (2011) 241602 [arXiv:1104.3850] [InSPIRE].

[15] A. Gadde, L. Rastelli, S.S. Razamat and W. Yan, Gauge theories and Macdonald polynomials, Commun. Math. Phys. 319 (2013) 147 [arXiv:1110.3740] [InSPIRE].

[16] T. Dimofte and S. Gukov, Refined, motivic and quantum, Lett. Math. Phys. 91 (2010) 1 [arXiv: 0904.1420] [INSPIRE].

[17] T. Dimofte, S. Gukov and Y. Soibelman, Quantum wall crossing in $N=2$ gauge theories, Lett. Math. Phys. 95 (2011) 1 [arXiv:0912.1346] [INSPIRE].

[18] S. Cecotti, A. Neitzke and C. Vafa, Twistorial topological strings and a tt* geometry for $N=2$ theories in 4d, Adv. Theor. Math. Phys. 20 (2016) 193 [arXiv:1412.4793] [InSPIRE].

[19] C. Closset, T.T. Dumitrescu, G. Festuccia and Z. Komargodski, Supersymmetric field theories on three-manifolds, JHEP 05 (2013) 017 [arXiv:1212.3388] [INSPIRE].

[20] T. Dimofte, D. Gaiotto and S. Gukov, 3-manifolds and 3d indices, Adv. Theor. Math. Phys. 17 (2013) 975 [arXiv:1112.5179] [INSPIRE].

[21] D. Gang, E. Koh and K. Lee, Line operator index on $S^{1} \times S^{3}$, JHEP 05 (2012) 007 [arXiv: 1201.5539] [INSPIRE].

[22] A.D. Shapere and Y. Tachikawa, Central charges of $N=2$ superconformal field theories in four dimensions, JHEP 09 (2008) 109 [arXiv:0804.1957] [INSPIRE]. 
[23] C.M. Ringel, Tame algebras and integral quadratic forms, Lect. Notes Math. 1099 (1984) 1.

[24] M. Bershadsky, A. Johansen, V. Sadov and C. Vafa, Topological reduction of $4 D S Y M$ to $2 D$ $\sigma$-models, Nucl. Phys. B 448 (1995) 166 [hep-th/9501096] [INSPIRE].

[25] A. Kapustin, Holomorphic reduction of $N=2$ gauge theories, Wilson-' $t$ Hooft operators and S-duality, hep-th/0612119 [INSPIRE].

[26] P. Putrov, J. Song and W. Yan, (0,4) dualities, JHEP 03 (2016) 185 [arXiv:1505.07110] [INSPIRE].

[27] A. Gadde, S.S. Razamat and B. Willett, On the reduction of $4 d N=1$ theories on $S^{2}$, JHEP 11 (2015) 163 [arXiv:1506.08795] [INSPIRE].

[28] L. Bhardwaj and Y. Tachikawa, Classification of $4 d N=2$ gauge theories, JHEP 12 (2013) 100 [arXiv:1309.5160] [INSPIRE].

[29] E. Witten, On the conformal field theory of the Higgs branch, JHEP 07 (1997) 003 [hep-th/9707093] [INSPIRE].

[30] F. Benini, R. Eager, K. Hori and Y. Tachikawa, Elliptic genera of two-dimensional $N=2$ gauge theories with rank-one gauge groups, Lett. Math. Phys. 104 (2014) 465 [arXiv: 1305.0533] [INSPIRE].

[31] F. Benini, R. Eager, K. Hori and Y. Tachikawa, Elliptic genera of $2 d N=2$ gauge theories, Commun. Math. Phys. 333 (2015) 1241 [arXiv:1308.4896] [INSPIRE].

[32] A. Gadde and S. Gukov, 2d index and surface operators, JHEP 03 (2014) 080 [arXiv: 1305.0266] [INSPIRE].

[33] F. Benini and A. Zaffaroni, A topologically twisted index for three-dimensional supersymmetric theories, JHEP 07 (2015) 127 [arXiv:1504.03698] [INSPIRE].

[34] D. Gaiotto, G.W. Moore and A. Neitzke, Four-dimensional wall-crossing via three-dimensional field theory, Commun. Math. Phys. 299 (2010) 163 [arXiv:0807.4723] [INSPIRE].

[35] G. 't Hooft, On the phase transition towards permanent quark confinement, Nucl. Phys. B 138 (1978) 1 [INSPIRE].

[36] G. 't Hooft, A property of electric and magnetic flux in non-Abelian gauge theories, Nucl. Phys. B 153 (1979) 141 [INSPIRE].

[37] G. 't Hooft, Topology of the gauge condition and new confinement phases in non-Abelian gauge theories, Nucl. Phys. B 190 (1981) 455 [INSPIRE].

[38] E. Witten, Dyons of charge e $\theta / 2 \pi$, Phys. Lett. B 86 (1979) 283 [inSPIRE].

[39] M. Alim, S. Cecotti, C. Cordova, S. Espahbodi, A. Rastogi and C. Vafa, $N=2$ quantum field theories and their BPS quivers, Adv. Theor. Math. Phys. 18 (2014) 27 [arXiv:1112.3984] [INSPIRE].

[40] S. Cecotti, Categorical tinkertoys for $N=2$ gauge theories, Int. J. Mod. Phys. A 28 (2013) 1330006 [arXiv: 1203.6734] [INSPIRE].

[41] L.D. Faddeev and A. Yu. Volkov, Abelian current algebra and the Virasoro algebra on the lattice, Phys. Lett. B 315 (1993) 311 [hep-th/9307048] [INSPIRE].

[42] S. Cecotti and M. Del Zotto, On Arnold's 14 'exceptional' $N=2$ superconformal gauge theories, JHEP 10 (2011) 099 [arXiv:1107.5747] [INSPIRE]. 
[43] M. Alim, S. Cecotti, C. Cordova, S. Espahbodi, A. Rastogi and C. Vafa, BPS quivers and spectra of complete $N=2$ quantum field theories, Commun. Math. Phys. 323 (2013) 1185 [arXiv: 1109.4941] [INSPIRE].

[44] S. Cecotti and M. Del Zotto, The BPS spectrum of the $4 d \mathrm{~N}=2 S C F T$ 's $H_{1}, H_{2}, D_{4}, E_{6}$, $E_{7}, E_{8}, J H E P 06$ (2013) 075 [arXiv: 1304.0614] [INSPIRE].

[45] S. Cecotti and M. Del Zotto, Galois covers of $N=2$ BPS spectra and quantum monodromy, Adv. Theor. Math. Phys. 20 (2016) 1227 [arXiv:1503.07485] [InSPIRE].

[46] H. Meltzer, Exceptional vector bundles, tilting sheaves and tilting complexes for weighted projective lines, American Mathematical Soc., U.S.A., (2004).

[47] S. Cecotti and M. Del Zotto, Higher S-dualities and Shephard-Todd groups, JHEP 09 (2015) 035 [arXiv: 1507.01799] [INSPIRE].

[48] S. Cecotti and M. Del Zotto, $Y$ systems, $Q$ systems and $4 D N=2$ supersymmetric QFT, J. Phys. A 47 (2014) 474001 [arXiv:1403.7613] [InSPIRE].

[49] W. Nahm, Conformal field theory and torsion elements of the Bloch group, hep-th/0404120 [INSPIRE].

[50] K. Hori, A. Iqbal and C. Vafa, D-branes and mirror symmetry, hep-th/0005247 [INSPIRE].

[51] S. Keegan and W. Nahm, Nahm's conjecture and coset models, arXiv:1103.4986 [INSPIRE].

[52] S. Cecotti and C. Vafa, Classification of complete $N=2$ supersymmetric theories in 4 dimensions, Surv. Diff. Geom. 18 (2013) 1 [arXiv:1103.5832] [INSPIRE].

[53] C. Beem, M. Lemos, P. Liendo, L. Rastelli and B.C. van Rees, The $N=2$ superconformal bootstrap, JHEP 03 (2016) 183 [arXiv: 1412.7541] [INSPIRE].

[54] B.L. Feigin and A.V. Stoyanovsky, Quasi-particles models for the representation of Lie algebras and geometry of flag manifold, Funct. Anal. Appl. 28 (1994) 68 [hep-th/9308079] [INSPIRE].

[55] K. Garrett, M.E. Ismail and D. Stanton, Variants of the Rogers-Ramanujan identities, Adv. Appl. Math. 23 (1999) 274.

[56] B.L. Feigin, T. Nakanishi and H. Ooguri, The annihilating ideals of minimal models, Int. J. Mod. Phys. A 7 (1992) 217 [INSPIRE].

[57] W. Nahm, A. Recknagel and M. Terhoeven, Dilogarithm identities in conformal field theory, Mod. Phys. Lett. A 8 (1993) 1835 [hep-th/9211034] [INSPIRE].

[58] J. Song, Superconformal indices of generalized Argyres-Douglas theories from $2 d$ TQFT, JHEP 02 (2016) 045 [arXiv: 1509.06730] [INSPIRE].

[59] S.O. Warnaar and W. Zudilin, Dedekind's $\eta$-function and Rogers-Ramanujan identities, Bull. Lond. Math. Soc. 44 (2012) 1 [arXiv:1001.1571].

[60] J.-F. Fortin, P. Mathieu and S.O. Warnaar, Characters of graded parafermion conformal field theory, Adv. Theor. Math. Phys. 11 (2007) 945 [hep-th/0602248] [INSPIRE].

[61] O. Warnaar, unpublished notes.

[62] M. Buican and T. Nishinaka, On the superconformal index of Argyres-Douglas theories, J. Phys. A 49 (2016) 015401 [arXiv:1505.05884] [INSPIRE].

[63] M. Buican and T. Nishinaka, Argyres-Douglas theories, the Macdonald index and an RG inequality, JHEP 02 (2016) 159 [arXiv: 1509.05402] [INSPIRE]. 
[64] F. Benini and N. Bobev, Two-dimensional SCFTs from wrapped branes and c-extremization, JHEP 06 (2013) 005 [arXiv: 1302.4451] [INSPIRE].

[65] A. Gadde, S. Gukov and P. Putrov, Fivebranes and 4-manifolds, arXiv:1306.4320 [INSPIRE].

[66] I. Bah and V. Stylianou, Gravity duals of $N=(0,2)$ SCFTs from M5-branes, arXiv: 1508.04135 [INSPIRE].

[67] D. Xie, General Argyres-Douglas theory, JHEP 01 (2013) 100 [arXiv:1204.2270] [INSPIRE].

[68] D. Xie and P. Zhao, Central charges and RG flow of strongly-coupled $N=2$ theory, JHEP 03 (2013) 006 [arXiv: 1301.0210] [INSPIRE].

[69] A.N. Kirillov, Dilogarithm identities, partitions and spectra in conformal field theory. 1, hep-th/9212150 [INSPIRE].

[70] G.E. Andrews and B.C. Berndt, Ramanujan's lost notebook. Part II, Springer, New York U.S.A., (2009).

[71] G.E. Andrews and B.C. Berndt, Ramanujan's lost notebook. Part I, Springer, New York U.S.A., (2005).

[72] G. Gasper and M. Rahman, Basic hypergeometric series, Encyclopedia of Mathematics and its Applications, vol. 96, Cambridge University Press, Cambridge U.K., (2004).

[73] G.E. Andrews, An analytic generalization of the Rogers-Ramanujan identities for odd moduli, Proc. Nat. Acad. Sci. 71 (1974) 4082.

[74] G. Andrews, Multiple series Rogers-Ramanujan type identities, Pacific J. Math. 114 (1984) 267. 